\title{
The Nirenberg problem on high dimensional half spheres: the effect of pinching conditions
}

\author{
Mohameden Ahmedou ${ }^{1}$ (D) Mohamed Ben Ayed $^{2}$
}

Received: 23 December 2020 / Accepted: 11 May 2021 / Published online: 2 July 2021

(c) The Author(s) 2021

Abstract

In this paper we study the Nirenberg problem on standard half spheres $\left(\mathbb{S}_{+}^{n}, g\right), n \geq 5$, which consists of finding conformal metrics of prescribed scalar curvature and zero boundary mean curvature on the boundary. This problem amounts to solve the following boundary value problem involving the critical Sobolev exponent:

$$
\text { (P) } \begin{cases}-\Delta_{g} u+\frac{n(n-2)}{4} u=K u^{\frac{n+2}{n-2}}, u>0 & \text { in } \mathbb{S}_{+}^{n}, \\ \frac{\partial u}{\partial v}=0 & \text { on } \partial \mathbb{S}_{+}^{n}\end{cases}
$$

where $K \in C^{3}\left(\mathbb{S}_{+}^{n}\right)$ is a positive function. This problem has a variational structure but the related Euler-Lagrange functional $J_{K}$ lacks compactness. Indeed it admits critical points at infinity, which are limits of non compact orbits of the (negative) gradient flow. Through the construction of an appropriate pseudogradient in the neighborhood at infinity, we characterize these critical points at infinity, associate to them an index, perform a Morse type reduction of the functional $J_{K}$ in their neighborhood and compute their contribution to the difference of topology between the level sets of $J_{K}$, hence extending the full Morse theoretical approach to this non compact variational problem. Such an approach is used to prove, under various pinching conditions, some existence results for $(\mathcal{P})$ on half spheres of dimension $n \geq 5$.

Mathematics Subject Classification $35 \mathrm{C} 60 \cdot 58 \mathrm{~J} 60 \cdot 53 \mathrm{C} 21$

Dedicated to the memory of Prof. Louis Nirenberg

Communicated by A. Malchiodi.

Mohameden Ahmedou

Mohameden.Ahmedou@math.uni-giessen.de

Mohamed Ben Ayed

Mohamed.Benayed@fss.rnu.tn

1 Mathematisches Institut der Justus-Liebig-Universität Giessen, Arndtsrasse 2, 35392 Giessen, Germany

2 Département de Mathématiques, Faculté des Sciences de Sfax, Université de Sfax, Route de Soukra, BP. 1171, Sfax 3000, Tunisia 


\section{Contents}

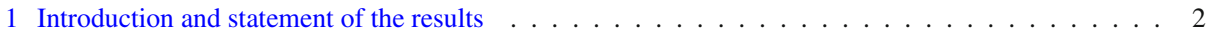

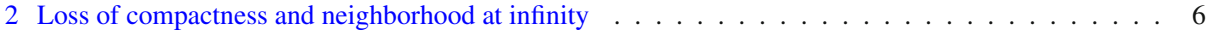

3 Pseudogradient and Morse Lemma at infinity . . . . . . . . . . . . . . . . . . . . 9

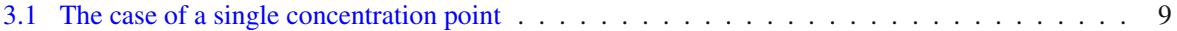

3.2 The case of multiple concentration points . . . . . . . . . . . . . . . . . . . . 12

3.2.1 Construction of some local pseudogradients . . . . . . . . . . . . . . . . . 13

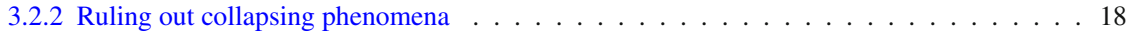

3.2 .3 Ruling out bubble towers phenomena . . . . . . . . . . . . . . . . 23

3.2.4 Critical points at infinity and their topological contribution . . . . . . . . . . . . . 27

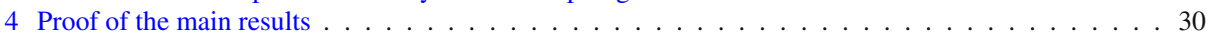

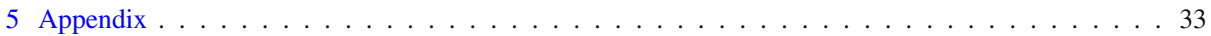

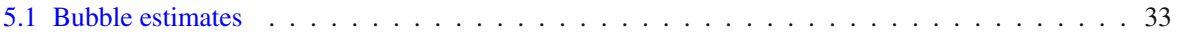

5.2 Asymptotic expansion of the functional and its gradient . . . . . . . . . . . . . . . 35

5.3 Counting index formulae . . . . . . . . . . . . . . . . . . . . . 38

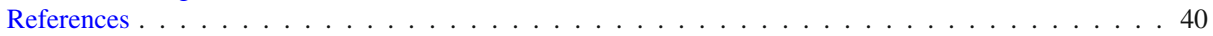

\section{Introduction and statement of the results}

In the early seventieth of the last century Louis Nirenberg asked the following question: Can a smooth positive function $K \in C^{\infty}\left(\mathbb{S}^{n}\right)$ defined on the standard $n$-dimensional sphere $\left(\mathbb{S}^{n}, g\right)$ be realized as the scalar curvature of a metric $\bar{g}$ conformally equivalent to $g$ ?

On $\mathbb{S}^{2}$, setting $\bar{g}=e^{2 u} g$ the Nirenberg problem is equivalent to solving the following nonlinear elliptic equation

$$
-\Delta_{g} u+1=K e^{2 u}, \text { in } \mathbb{S}^{2},
$$

where $\Delta_{g}$ denotes the Laplace Beltrami operator.

For spheres of dimensions $n \geq 3$ and writing the conformal metric as $\bar{g}:=u^{4 /(n-2)} g$, the Nirenberg problem amounts to solve the following nonlinear elliptic equation involving the Sobolev critical exponent:

$$
(\mathcal{N P}) \quad-\Delta_{g} u+\frac{n(n-2)}{4} u=K u^{\frac{n+2}{n-2}} ; \quad u>0, \quad \text { in } \mathbb{S}^{n}
$$

The Nirenberg problem has attracted a lot attention in the last half century. See $[3,4,6,7$, 10-12,19-24,28,31,32,36,37] and the references therein. Actually due to Kazdan-Warner obstructions, see $[18,28]$, a positive answer to the Nirenberg's question requires imposing conditions on the function $K$. It turns out that finding sufficient conditions under which the Nirenberg problem is solvable depends strongly on the dimension $n$ and the behavior of the function $K$ near its critical points. Indeed in low dimension $n<5$ index counting criteria have been obtained, see [7,20,27,31,32]. Such a counting index criterium fails, under the nondegeneracy assumption $(N D)$ (that is $\Delta K \neq 0$ at critical points of $K$ ), if the dimension $n \geq 5$. They can be extended on high dimensional spheres in the perturbative setting (that is when $K$ is close to a constant) see [19,24] or under some flatness assumptions see [16,22,31]. To explain the main difficulty in studying the Nirenberg problem and the differences between the low dimensional case $n<5$ and the high dimensional one $n \geq 5$, we point out that due to the presence of the Sobolev critical exponent, the corresponding Euler-Lagrange functional does not satisfy the Palais-Smale condition. One way to overcome such a difficulty is to consider the following subcritical approximation of the problem $(\mathcal{N P})$ :

$$
\left(\mathcal{N} \mathcal{P}_{\varepsilon}\right)-\Delta_{g} u+\frac{n(n-2)}{4} u=K u^{\frac{n+2}{n-2}-\varepsilon}, \quad u>0 \text { in } \mathbb{S}^{n},
$$


where $\varepsilon>0$ is a small parameter. In this way one recovers the compactness and one then studies the behavior of blowing up solution $u_{\varepsilon}$ of $\left(\mathcal{N} \mathcal{P}_{\varepsilon}\right)$ as the parameter $\varepsilon$ goes to zero. Actually it can be proved that finite energy blowing up solutions of $\left(\mathcal{N} \mathcal{P}_{\varepsilon}\right)$ can have only isolated simple blow up points which are critical points of the function $K$, see $[23,31,32,35]$. The reason of the additional difficulty in the high dimensional case lies in the complexity of the blow up phenomenon. Indeed in dimensions $n=2,3$ there are only single blow up points, see, [7,20,27,31,37] and in dimension $n=4$ multiple bubbling may occur only under some extra condition, see $[11,32]$ while, under the non degeneracy assumption $(N D)$, on spheres of dimension $n \geq 5$ every $m$-tuple $\left(q_{1}, \ldots, q_{m}\right)$ of distinct critical points of $K$, satisfying $\Delta K\left(q_{i}\right)<0$ for each $i=1, \ldots, m$ can be realized as a concentration set of blowing up solutions of $\left(\mathcal{N} \mathcal{P}_{\varepsilon}\right)$. See [34].

Regarding the high dimensional case $n \geq 5$, Malchiodi and Mayer [35] obtained recently an interesting existence criterium under some pinching condition. Their result reads as follows:

Theorem A [35] Let $n \geq 5$ and $K \in C^{\infty}\left(\mathbb{S}^{n}\right)$ be a positive Morse function satisfying the following conditions

(i)

$$
\forall q \in \mathbb{S}^{n}, \quad \nabla K(q)=0 \Rightarrow \Delta K(q) \neq 0,
$$

(ii)

$$
K_{\max } / K_{\min } \leq(3 / 2)^{1 /(n-2)},
$$

where $K_{\max }:=\max _{\mathbb{S}^{n}} K$ and $K_{\min }:=\min _{\mathbb{S}^{n}} K$

(iii)

$$
\#\left\{q \in \mathbb{S}^{n} ; \nabla K(q)=0 ; \Delta K(q)<0\right\} \geq 2,
$$

where \#A denotes the cardinal of the set A.

Then Nirenberg Problem $(\mathcal{N P})$ has at least one solution.

In this paper we consider a version of the Nirenberg problem on standard half spheres $\left(\mathbb{S}_{+}^{n}, g\right)$. Namely we prescribe simultaneously the scalar curvature to be a positive function $0<K \in C^{3}\left(\mathbb{S}_{+}^{n}\right)$ and the boundary mean curvature to be zero. This amounts to solve the following boundary value problem

$$
\text { (P) } \begin{cases}-\Delta_{g} u+\frac{n(n-2)}{4} u=K u^{(n+2) /(n-2)}, u>0 & \text { in } \mathbb{S}_{+}^{n}, \\ \frac{\partial u}{\partial v}=0 & \text { on } \partial \mathbb{S}_{+}^{n},\end{cases}
$$

where $K \in C^{3}\left(\mathbb{S}_{+}^{n}\right)$ is a positive function.

This problem has been studied on half spheres of dimensions $n=2,3$, 4. See the papers $[13-15,17,25,29,30]$ and the references therein. Very much like the case of spheres, to recover compactness one considers here the following subcritical approximation

$$
\left(\mathcal{P}_{\varepsilon}\right) \begin{cases}-\Delta_{g} u+\frac{n(n-2)}{4} u=K u^{\frac{n+2}{n-2}-\varepsilon}, u>0 & \text { in } \mathbb{S}_{+}^{n}, \\ \frac{\partial u}{\partial v}=0 & \text { on } \partial \mathbb{S}_{+}^{n} .\end{cases}
$$

Just as above, there are two alternatives for the behavior of a sequence of solutions $u_{\varepsilon}$ of $\left(\mathcal{P}_{\varepsilon}\right)$. Either the $\left\|u_{\varepsilon}\right\|_{L^{\infty}}$ remains uniformly bounded or it blows up and if it does $u_{\varepsilon}^{2 n /(n-2)} \mathcal{L}^{n}$ (where $\mathcal{L}^{n}$ denotes the Lebesgue measure) converges to a sum of Dirac masses, some of them are sitting in the interior and the others ones are located on the boundary. The interior points are critical points of $K$ satisfying that $\Delta K \leq 0$ and the boundary points are critical points 
of $K_{1}$ the restriction of $K$ on the boundary and satisfying that $\partial K / \partial v \geq 0$. See [14,17,25]. Furthermore a refined blow up analysis, under the non degeneracy assumption that $\Delta K \neq 0$ at interior critical points of $K$ and that $\partial K / \partial v \neq 0$ at critical points of $K_{1}$, shows that in the dimension $n=3$ multiple bubbling may occur but all blow up points are isolated simple, see $[25,30]$. Moreover in dimensions $n=2,3$ counting index criteria have been established, see $[14,17,25,29]$. Furthermore under additional condition on $K_{1}$ it has been proved in [15] that all blow up points are isolated simple, but already in dimension $n=4$ counting index formulae, under the above non degeneracy conditions fail. More surprisingly and in contrast with the case of closed spheres, the Nirenberg problem on half spheres may have non simple blow up points, even for finite energy bubbling solutions of $\left(\mathcal{P}_{\varepsilon}\right)$ see [1,2].

In this paper we study Problem $(\mathcal{P})$ from the viewpoint of the theory of critical points at infinity. In this approach initiated by the late Bahri, see [5-8], one studies the possible ends of non compact orbits of the (negative) gradient of the associated Euler Lagrange functional. The method consists of taking advantage of the concentration-compactness analysis of non converging Palais-Smale sequences to identify a potential neighborhood at infinity where concentration may occur. Then one constructs a global pseudogradient for which the full analysis of the $\omega$-limit set, in this neighborhood is easier than for the genuine gradient flow and then uses it to characterize critical points at infinity. One then performs a Morse reduction near these critical points at infinity in order to compute their topological contribution to the difference of topology between the level sets of the Euler-Lagrange functional.

Before stating our main results, we set up some notation and introduce our assumptions.

For the function $K$ and its restriction on the boundary $K_{1}:=K_{\left\lfloor\partial \mathbb{S}^{n}\right.}$, we use the following assumption:

(H1): We assume that $K$ is a $C^{3}\left(\overline{\mathbb{S}_{+}^{n}}\right)$ positive function, which has only non-degenerate critical points with $\Delta K \neq 0$. (We point out that some of these points can be on the boundary.)

(H2): We assume that the restriction of $K$ on the boundary $K_{1}:=K_{\mid \partial \mathbb{S}_{+}^{n}}$ has only nondegenerate critical points $z$ 's. Furthermore we assume that if $z$ is not a local maximum point of $K_{1}$, we have that $\partial K / \partial v(z) \leq 0$.

(H3): If $z \in \partial \mathbb{S}_{+}^{n}$ is a critical point of $K_{1}$ satisfying that $\partial K / \partial v(z)=0$, hence $z$ is actually a critical point of $K$ on $\partial \mathbb{S}_{+}^{n}$, we assume that $\Delta K(z) \neq 0$ and one of the following conditions is satisfied:

(i) either $\partial K / \partial v(a) \Delta K(z) \leq 0$ for each $a \in \partial \mathbb{S}_{+}^{n}$ in a small neighborhood of $z$,

(ii) or $\lim _{a \in \partial \mathbb{S}_{+}^{n} ; a \rightarrow z} \frac{\partial K / \partial v(a)}{d(a, z)}=0$.

Next we introduce the following subsets of critical points of $K$ and $K_{1}$

$$
\begin{aligned}
\mathcal{K}_{i n}^{-} & :=\left\{y \in \mathbb{S}_{+}^{n}: \nabla K(y)=0 \text { and } \Delta K(y)<0\right\}, \\
\mathcal{K}_{b}^{+} & :=\left\{z \in \partial \mathbb{S}_{+}^{n}: \nabla K_{1}(z)=0 \text { and } \partial K / \partial v(z)>0\right\}, \\
\mathcal{K}_{b}^{0,-} & :=\left\{z \in \partial \mathbb{S}_{+}^{n}: \nabla K_{1}(z)=0 ; \partial K / \partial v(z)=0 \text { and } \Delta K(z)<0\right\} .
\end{aligned}
$$

Furthermore we define

$$
\mathcal{K}^{\infty}:=\mathcal{K}_{i n}^{-} \cup \mathcal{K}_{b}^{+} \cup \mathcal{K}_{b}^{0,-} .
$$

Our first result is an existence result under a pinching assumption, which parallels the above mentioned existence result of Malchiodi-Mayer. Namely we prove

Theorem 1.1 Let $n \geq 5$ and $0<K \in C^{3}\left(\overline{\mathbb{S}_{+}^{n}}\right)$ satisfying the assumptions (H1), (H2) and (H3).

$$
\text { If the following conditions hold }
$$


(i)

$$
K_{\max } / K_{\min }<(5 / 4)^{1 /(n-2)},
$$

where $K_{\max }:=\max _{\mathbb{S}_{+}^{n}} K$ and $K_{\min }:=\min _{\mathbb{S}_{+}^{n}} K$.

(ii)

$$
\# \mathcal{K}^{\infty} \geq 2
$$

where \#A denotes the cardinal of the set A. Then Problem $(\mathcal{P})$ has at least one solution.

Remark 1.2 1. The above theorem is the counterpart of the existence result of MalchiodiMayer [35](see Theorem A quoted above). We point that the proof of Theorem 1.1, compared with the proof of Theorem A is more involved. In particular the counting index argument in our case is more subtle. Indeed due to the influence of the boundary the blow up picture is more complicated. Namely we have boundary and interior blow up as well as mixed configurations involving both of them. Such a complicated picture imposes to consider 4 critical levels instead of two critical levels needed in the case of closed spheres. Such a fact makes the index counting of the associated critical points at infinity more involved, see Lemmas 5.9, 5.8 in the "Appendix".

2. The conditions $(H 2),(H 3)$ are used to rule out non simple blow up, see [1]. A phenomenon which does not occur in the case of closed spheres. See Sect. 3.2.2.

The above pinching condition $(i)$ of Theorem 1.1 can be relaxed when combined with some counting index formula involving either the boundary blow up points or the interior blow points. In the next theorem we provide an existence result involving the boundary blow up points. Namely we prove:

Theorem 1.3 Let $n \geq 5$ and $0<K \in C^{3}\left(\overline{\mathbb{S}_{+}^{n}}\right)$. Assume that the critical points of $K_{1}:=K_{\mid \partial \mathbb{S}_{+}^{n}}$ are non degenerate and that $K$ satisfies the assumption (H3). If the following conditions hold (a)

$$
K_{\max } / K_{\min }<2^{1 /(n-2)},
$$

(b)

$$
A_{1}:=\sum_{z \in \mathcal{K}_{b}^{+} \cup \mathcal{K}_{b}^{0,-}}(-1)^{n-1-\operatorname{morse}\left(K_{1}, z\right)} \neq 1 .
$$

Then Problem $(\mathcal{P})$ has at least one solution.

Next we assume that the above index formula $A_{1}=1$, which implies, in particular that the number of boundary blow up points is an odd number, say $2 k+1$, where $k \in \mathbb{N}_{0}$.

The next existence result combined a pinching condition with a counting index formulae involving interior blow up points. Namely we prove:

Theorem 1.4 Let $n \geq 5$ and $0<K \in C^{3}\left(\overline{\mathbb{S}_{+}^{n}}\right)$ satisfying the assumptions $(H 1)$, (H2) and (H3).

If the following conditions hold

(i)

$$
K_{\max } / K_{\min }<(3 / 2)^{1 /(n-2)} \text { and } A_{1}=1,
$$

where $A_{1}$ is defined in Theorem 1.3, 
(ii)

$$
B_{1}:=\sum_{y \in \mathcal{K}_{i n}^{-}}(-1)^{n-\operatorname{morse}(K, y)} \neq-k,
$$

where $\#\left(\mathcal{K}_{b}^{+} \cup \mathcal{K}_{b}^{0,-}\right)=2 k+1, k \in \mathbb{N}_{0}$. Then Problem $(\mathcal{P})$ has at least one solution.

Regarding the method of proof of our main existence results, Theorems 1.1, 1.3 and 1.4 some comments are in order. Indeed although the general scheme falls in the framework of the techniques and ideas of the critical point theory at infinity, see $[6,7,11]$, the main arguments here are of a different flavor. Indeed with respect to the case of closed spheres, treated by A.Bahri in his seminal paper [6], the case of half spheres presents new aspects: From one part the blow up picture is more complicated (interior, boundary and mixed configurations) and from another part the behavior of the self interactions of interior bubbles and boundary bubbles is drastically different. A fact which was used in [1] to construct subcritical solutions having non simple blow ups. To rule out such a possibility, under our assumption (H2) and (H3), we had to come up with a barycentric vector field which moves a cluster of concentration points towards their common barycenter and to prove that along the flow lines of such a vector field the functional decreases and the concentration rates of an initial value do not increase, see Lemma 3.9. Furthermore we prove that in the neighborhood of critical points at infinity, the concentration rates are comparable and the concentration points are not to close to each other. See Sects. 3.2.2 and 3.2.3.

The remainder of this paper is organized as follows: In Sect. 2 we set up the variational framework and define the neighborhood at infinity and in Sect. 3 we construct an appropriate pseudogradient in the vicinity of highly concentrated bubbles and derive from the analysis of the behavior of its flow lines the set of its critical points at infinity. Section 4 is devoted to the proof of the main existence results of this paper. Lastly we collect in the appendix some estimates of the bubble, fine asymptotic expansion of the Euler-Lagrange functional and its gradient in the neighborhood at infinity as well as useful counting index formula for the critical points of the function $K$ and its restriction $K_{1}$ on the boundary.

\section{Loss of compactness and neighborhood at infinity}

In this section we set up the analytical framework of the variational problem associated to the Nirenberg problem and recall the description of its lack of compactness. Let $H^{1}\left(\mathbb{S}_{+}^{n}\right)$ be the Sobolev space endowed with the norm

$$
\|u\|^{2}:=\int_{\mathbb{S}_{+}^{n}}|\nabla u|^{2}+\frac{n(n-2)}{4} \int_{\mathbb{S}_{+}^{n}} u^{2},
$$

and let $\Sigma$ denote its unit sphere.

Problem $(\mathcal{P})$ has a variational structure. Namely its solutions are in one to one correspondence with the critical points of the functional

$$
J_{K}(u):=\frac{\|u\|^{2}}{\left(\int_{\mathbb{S}_{+}^{n}} K|u|^{2 n /(n-2)}\right)^{(n-2) / n}} \quad \text { defined on } \Sigma^{+}:=\{u \in \Sigma ; u \geq 0\} .
$$

The functional $J_{K}$ fails to satisfy the Palais Smale condition. To describe non converging Palais-Smale sequences we introduce the following notation. 
For $a \in \overline{\mathbb{S}_{+}^{n}}$ and $\lambda>0$ we define the standard bubble to be

$$
\delta_{a, \lambda}(x):=c_{0} \frac{\lambda^{n-2 / 2}}{\left(\lambda^{2}+1+\left(1-\lambda^{2}\right) \cos d(a, x)\right)^{n-2 / 2}},
$$

where $d$ is the geodesic distance on $\mathbb{S}_{+}^{n}$ and $c_{0}$ is a constant chosen such that

$$
-\Delta \delta_{a, \lambda}+\frac{n(n-2)}{4} \delta_{a, \lambda}=\delta_{a, \lambda}^{(n+2) /(n-2)} \text { in } \mathbb{S}_{+}^{n} .
$$

For $a \in \overline{\mathbb{S}}_{+}^{n}$, we define projected bubble $\varphi_{a, \lambda}$ to be the unique solution of

$$
-\Delta \varphi_{a, \lambda}+\frac{n(n-2)}{4} \varphi_{a, \lambda}=\delta_{a, \lambda}^{(n+2) /(n-2)} \quad \text { in } \mathbb{S}_{+}^{n} ; \quad \frac{\partial \varphi_{a, \lambda}}{\partial v}=0 \text { on } \partial \mathbb{S}_{+}^{n} .
$$

We point out that $\varphi_{a, \lambda}=\delta_{a, \lambda}$ if $a \in \partial \mathbb{S}_{+}^{n}$.

Next for $m \in \mathbb{N}$ and $p, q \in \mathbb{N}_{0}$ such that $q+2 p=m$ we define the neighborhood of potential critical points at Infinity $V(m, q, p, \varepsilon)$ as follows:

$$
\begin{aligned}
& V(m, q, p, \varepsilon):=\left\{u \in \Sigma: \exists \lambda_{1}, \ldots, \lambda_{p+q}>\varepsilon^{-1} ; \exists a_{1}, \ldots, a_{q+p} \in \overline{\mathbb{S}_{+}^{n}}\right. \text {, with } \\
& \lambda_{i} d\left(a_{i}, \partial \mathbb{S}_{+}^{n}\right)<\varepsilon, \forall i \leq q \text {, and } \lambda_{i} d\left(a_{i}, \partial \mathbb{S}_{+}^{n}\right)>\varepsilon^{-1} \forall i>q, \\
& \left.\varepsilon_{i j}<\varepsilon \text { such that }\left\|u-\frac{\sum_{i=1}^{p+q} K\left(a_{i}\right)^{(2-n) / 4} \varphi_{a_{i}, \lambda_{i}}}{\left\|\sum_{i=1}^{p+q} K\left(a_{i}\right)^{(2-n) / 4} \varphi_{a_{i}, \lambda_{i}}\right\|}\right\|<\varepsilon\right\} \text {, }
\end{aligned}
$$

where

$$
\varepsilon_{i j}:=\left(\frac{\lambda_{i}}{\lambda_{j}}+\frac{\lambda_{j}}{\lambda_{i}}+2 \lambda_{i} \lambda_{j}\left(1-\cos \left(d\left(a_{i}, a_{j}\right)\right)\right)\right)^{2-n / 2} .
$$

In the following we describe non converging Palais-Smale sequences. Such a description, which is by now standard, follows from concentration-compactness arguments as in $[33,38]$ and reads as follows

Proposition 2.1 Let $u_{k} \in \Sigma^{+}$be a sequence such that $\nabla J_{K}\left(u_{k}\right) \rightarrow 0$ and $J_{K}\left(u_{k}\right)$ is bounded. If Problem $(\mathcal{P})$ does not have a solution, then there exist $m \in \mathbb{N}$ and $p, q \in \mathbb{N}$ with $q+2 p=m$, a sequence of positive real numbers $\varepsilon_{k} \downarrow 0$ as well as subsequence of $u_{k}$, still denoted $u_{k}$ such that $u_{k} \in V\left(m, q, p, \varepsilon_{k}\right)$.

Following Bahri and Coron, we consider for $u \in V(m, q, p, \varepsilon)$ the following minimization problem

$\operatorname{Min}\left\{\left\|u-\sum_{i=1}^{p+q} \alpha_{i} \varphi_{a_{i}, \lambda_{i}}\right\| ; \alpha_{i}>0, \lambda_{i}>0, a_{i} \in \partial \mathbb{S}_{+}^{n}, \forall i=1, \ldots, q ; a_{i} \in \mathbb{S}_{+}^{n}, \forall q+1 \leq i \leq q+p\right\}$.

We then have the following proposition whose proof is identical, up to minor modification to the one of Proposition 7 in [8]

Proposition 2.2 Foranym $\in \mathbb{N}$ there exists $\varepsilon_{m}>0$ such that if $\varepsilon<\varepsilon_{m}$ and $u \in V(m, q, p, \varepsilon)$ the minimization problem (5) has, up to permutation, a unique solution.

Hence it follows from Proposition 2.2 that every $u \in V(m, q, p, \varepsilon)$ can be written in a unique way as

$$
u=\sum_{i=1}^{q} \alpha_{i} \delta_{a_{i}, \lambda_{i}}+\sum_{i=q+1}^{p+q} \alpha_{i} \varphi_{a_{i}, \lambda_{i}}+v
$$


where

$$
a_{i} \in \partial \mathbb{S}_{+}^{n}, i=1, \ldots, q \text { and } a_{i} \in \mathbb{S}_{+}^{n}, i=q+1, \ldots, p+q,
$$

and $v \in H^{1}\left(\mathbb{S}_{+}^{n}\right)$ satisfying

$$
\left(V_{0}\right)\|v\|<\varepsilon, \quad<v, \psi>=0, \text { for } \psi \in \bigcup_{1 \leq i \leq q ; q+1 \leq j \leq q+p}\left\{\delta_{i}, \frac{\partial \delta_{i}}{\partial \lambda_{i}}, \frac{\partial \delta_{i}}{\partial a_{i}}, \varphi_{j}, \frac{\partial \varphi_{j}}{\partial \lambda_{j}}, \frac{\partial \varphi_{j}}{\partial a_{j}}\right\},
$$

where $\delta_{i}:=\delta_{a_{i}, \lambda_{i}}$ and $\varphi_{i}:=\varphi_{a_{i}, \lambda_{i}}$. In addition, the variables $\alpha_{i}$ 's satisfy

$$
\left|1-J(u)^{n /(n-2)} \alpha_{i}^{4 /(n-2)} K\left(a_{i}\right)\right|=o_{\varepsilon}(1) \text { for each } i .
$$

In the next lemma we deal with the $v$-part of $u \in V(m, q, p, \varepsilon)$ in order to prove, that its effect is negligible with the concentration phenomenon. Namely we prove:

Lemma 2.3 Let $n \geq 5$. For $\varepsilon>0$ small, there exists a $C^{1}$-map which, to each $(\alpha:=$ $\left.\left(\alpha_{1}, \ldots, \alpha_{p+q}\right), a:=\left(a_{1}, \ldots, a_{p+q}\right), \lambda:=\left(\lambda_{1}, \ldots, \lambda_{p+q}\right)\right)$, such that $u=\sum_{i=1}^{p+q} \alpha_{i} \varphi_{i} \in$ $V(m, q, p, \varepsilon)$, associates $\bar{v}=\bar{v}_{(\alpha, a, \lambda)}$ satisfying

$$
J_{K}\left(\sum_{i=1}^{p+q} \alpha_{i} \varphi_{a_{i}, \lambda_{i}}+\bar{v}\right)=\min \left\{J_{K}\left(\sum_{i=1}^{p+q} \alpha_{i} \varphi_{a_{i}, \lambda_{i}}+v\right), v \text { satisfies }\left(V_{0}\right)\right\} .
$$

Moreover, there exists $c>0$ such that the following holds

$$
\|\bar{v}\| \leq c \sum_{i=1}^{q+p} \frac{\left|\nabla K\left(a_{i}\right)\right|}{\lambda_{i}}+\frac{1}{\lambda_{i}^{2}}+\left\{\begin{array}{l}
\sum \varepsilon_{i j}^{\frac{n+2}{2(n-2)}}\left(\ln \varepsilon_{i j}^{-1}\right)^{\frac{n+2}{2 n}}+\sum_{i>q} \frac{\ln \left(\lambda_{i} d_{i}\right)}{\left(\lambda_{i} d_{i}\right)^{(n+2) / 2}} \text { if } n \geq 6, \\
\sum \varepsilon_{i j}\left(\ln \varepsilon_{i j}^{-1}\right)^{3 / 5}+\sum_{i>q} \frac{1}{\left(\lambda_{i} d_{i}\right)^{3}} \text { if } n=5 .
\end{array}\right.
$$

Proof The proof follows as in Proposition 3.1 in [12] (see also [9]). Indeed, easy computations imply that

$$
\begin{aligned}
& J_{K}(u+v)=J_{K}(u)-f(v)+(1 / 2) Q(v)+o\left(\|v\|^{2}\right) \quad \text { where } \\
& f(v):=\int_{\mathbb{S}_{+}^{n}} K u^{\frac{n+2}{n-2}} v \text { and } Q(v):=\|v\|^{2}-\frac{n+2}{n-2} \sum_{i=1}^{N} \int_{\mathbb{S}_{+}^{n}} \delta_{i}^{4 /(n-2)} v^{2} .
\end{aligned}
$$

Note that $Q$ is a positive definite quadratic form (see [5]) and we have that

$$
f(v)=\sum \alpha_{i}^{\frac{n+2}{n-2}} \int_{\mathbb{S}_{+}^{n}} K \varphi_{i}^{\frac{n+2}{n-2}} v+O\left(\sum_{i \neq j} \int_{\mathbb{S}_{+}^{n}} \sup \left(\varphi_{j}, \varphi_{i}\right)^{\frac{4}{n-2}} \inf \left(\varphi_{j}, \varphi_{i}\right)|v|\right) .
$$

Observe that, for $n \geq 6$, it follows that $4 /(n-2) \leq 1$. Hence, using Holder's inequality, we get

$$
\begin{aligned}
& \int_{\mathbb{S}_{+}^{n}} \sup \left(\varphi_{j}, \varphi_{i}\right)^{\frac{4}{n-2}} \inf \left(\varphi_{j}, \varphi_{i}\right)|v| \leq \int_{\mathbb{S}_{+}^{n}}\left(\varphi_{j} \varphi_{i}\right)^{\frac{n+2}{2(n-2)}}|v| \\
& \leq c\|v\|\left(\int_{\mathbb{S}_{+}^{n}}\left(\delta_{j} \delta_{i}\right)^{\frac{n}{n-2}}\right)^{\frac{n+2}{2 n}} \leq c\|v\| \varepsilon_{i j}^{\frac{n+2}{2(n-2)}}\left(\ln \varepsilon_{i j}^{-1}\right)^{\frac{n+2}{2 n}} \quad \text { if } n \geq 6 \\
& \int_{\mathbb{S}_{+}^{5}} \sup \left(\varphi_{j}, \varphi_{i}\right)^{4 / 3} \inf \left(\varphi_{j}, \varphi_{i}\right)|v| \leq c\|v\| \varepsilon_{i j}\left(\ln \varepsilon_{i j}^{-1}\right)^{3 / 5} \text { if } n=5
\end{aligned}
$$


For the other term, for $i \leq q$ (that is $a_{i} \in \partial \mathbb{S}_{+}^{n}$ ), using the fact that $\left\langle\delta_{i}, v\right\rangle=0$, we get

$$
\begin{aligned}
\int_{\mathbb{S}_{+}^{n}} K \delta_{i}^{\frac{n+2}{n-2}} v & =O\left(\left|\nabla K\left(a_{i}\right)\right| \int_{\mathbb{R}_{+}^{n}}\left|x-a_{i}\right| \delta_{i}^{\frac{n+2}{n-2}}|v|+\int_{\mathbb{R}_{+}^{n}}\left|x-a_{i}\right|^{2} \delta_{i}^{\frac{n+2}{n-2}}|v|\right) \\
& =O\left(\left(\frac{\left|\nabla K\left(a_{i}\right)\right|}{\lambda_{i}}+\frac{1}{\lambda_{i}^{2}}\right)\|v\|\right) .
\end{aligned}
$$

For $i>q$, using Lemma 5.1, we get

$$
\begin{aligned}
\int_{\mathbb{S}_{+}^{n}} K \varphi_{i}^{\frac{n+2}{n-2}} v & =\int_{\mathbb{S}_{+}^{n}} K \delta_{i}^{\frac{n+2}{n-2}} v+O\left(\int_{\mathbb{S}_{+}^{n}} \delta_{i}^{\frac{4}{n-2}}\left|\varphi_{i}-\delta_{i}\right||v|\right) \\
& =O\left(\left(\frac{\left|\nabla K\left(a_{i}\right)\right|}{\lambda_{i}}+\frac{1}{\lambda_{i}^{2}}\right)\|v\|\right)+\left\{\begin{array}{l}
O\left(\|v\| /\left(\lambda_{i} d_{i}\right)^{n-2}\right) \text { if } n=5, \\
O\left(\|v\| \ln \left(\lambda_{i} d_{i}\right) /\left(\lambda_{i} d_{i}\right)^{(n+2) / 2}\right) \text { if } n \geq 6
\end{array}\right.
\end{aligned}
$$

and the result follows.

\section{Pseudogradient and Morse Lemma at infinity}

This section is devoted to the construction of a pseudogradient for the functional $J_{K}$, which has the property that along its flow lines there could be only finitely many isolated blow up ponits. Such a pseudogradient coincides with the gradient outside of $\bigcup_{m, q, p} V(m, q, p, \varepsilon / 2)$ and satisfies the Palais-Smale condition there. Moreover in each $V(m, q, p, \varepsilon)$ it has the property to move the concentration points according to $\nabla K$ or $\nabla K_{1}$, the $\alpha_{i}$ 's to their maximum values and the concentration $\lambda_{i}$ 's are moved so that the functional $J_{K}$ decreases along its flow lines. The global vector field is then defined by convex combining these two vector fields. Such a construction is then used to perform a Morse reduction near the singularities of the pseudogradient and to compute the difference of topology induced by the critical points at infinity between the level sets of the Euler-Lagrange functional $J_{K}$.

The first step in the construction of the pseudogradient is to describe the movement of the variable $v$. In fact, since $\bar{v}$ minimizes $J_{K}$ in the $v$-space, it follows from the classical Morse Lemma that there exists a change of variable $v \rightarrow V$ such that

$$
J_{K}\left(\sum_{i=1}^{p+q} \alpha_{i} \varphi_{a_{i}, \lambda_{i}}+v\right)=J_{K}\left(\sum_{i=1}^{p+q} \alpha_{i} \varphi_{a_{i}, \lambda_{i}}+\bar{v}\right)+\|V\|^{2}
$$

Hence, for the variable $V$, we will use $\dot{V}=-V$ to bring it to 0 . Thus, we need to construct some vector fields by moving the variables $\alpha_{i}, a_{i}$ and $\lambda_{i}$.

\subsection{The case of a single concentration point}

We point out that the construction of a pseudogradient satisfying the above properties becomes quite involved in the case of more than one concentration point. Indeed in the case of two bubbles sitting at different points, their mutual interaction comes into play. For this reason we start by constructing the needed pseudogradient in neighborhoods at infinity, containing one interior or one boundary point. To do so we consider two cases, the first one corresponds to $p=1$ and $q=0$ (case of an interior concentration point) and the second one corresponds to $p=0$ and $q=1$ (the case of a boundary point). Namely we prove: 
Proposition 3.1 Assume that $K$ satisfies $(H 1)$ and $(H 3)$ and that the critical points of $K_{1}$ are non-degenerate. A pseudogradient $W$ can be defined so that the following holds: There is a constant $c>0$ independent of $u=\alpha \varphi_{a, \lambda} \in V(2 p+q, q, p, \varepsilon)($ with $q=1$ or $p=1)$ such that

(i) $\left\langle-\nabla J_{K}(u), W\right\rangle \geq c\left\{\begin{array}{l}1 / \lambda^{2}+1 /(\lambda d)^{n-2}+|\nabla K(a)| / \lambda \text { if } p=1 ; q=0, \\ 1 / \mu+\left|1-J(u)^{\frac{n}{n-2}} \alpha^{\frac{4}{n-2}} K(a)\right| \text { if } p=0 ; q=1,\end{array}\right.$

(ii) $\left\langle-\nabla J_{K}(u+\bar{v}), W+\frac{\partial \bar{v}}{\partial(\alpha, a, \lambda)}(W)\right\rangle \geq c\left\{\begin{array}{l}1 / \lambda^{2}+1 /(\lambda d)^{n-2}+|\nabla K(a)| / \lambda \text { if } p=1 ; q=0, \\ 1 / \mu+\left|1-J(u)^{\frac{n}{n-2}} \alpha^{\frac{4}{n-2}} K(a)\right| \text { if } p=0 ; q=1,\end{array}\right.$

where $d:=d\left(a, \partial \mathbb{S}_{+}^{n}\right)$ for $a \in \mathbb{S}_{+}^{n}$ and $\mu^{-1}=|\nabla K(a)| / \lambda+1 / \lambda^{2}$ for $a \in \partial \mathbb{S}_{+}^{n}$.

(iii) The vector field $W$ is bounded with the property that along its flow lines, $\lambda$ increases only in the following region

- If $p=1$ then $\lambda$ increases if and only if the point a belongs to a small neighborhood of a critical point $y \in \mathbb{S}_{+}^{n}$ of $K$, such that $\Delta K(y)<0$

- If $q=1$ then $\lambda$ increases if and only if the point a belongs to a small neighborhood of a critical point $z \in \partial \mathbb{S}_{+}^{n}$ of $K_{1}$ such that either $(\partial K / \partial v)(z)>0$ or $(\partial K / \partial v)(z)=0$ and $\Delta K(z)<0$.

Proof We start by giving the proof of Claim $(i)$ for the case where $p=1$ and $q=0$ that is in $V(2,0,1, \varepsilon)$. First, we notice that, if $a$ is close to a critical point $y$ of $K$ in $\mathbb{S}_{+}^{n}$, then $\Delta K(a)=\Delta K(y)(1+o(1))$ and therefore $\Delta K(a)$ has a constant sign.

Let $M$ be a large constant and let $\psi_{1}$ be a $C^{\infty}$ cut off function defined by $\psi \in[0,1]$, $\psi_{1}(t)=1$ if $t \geq 2$ and $\psi_{1}(t)=0$ if $t \leq 1$. We define

$$
\begin{gathered}
W:=\psi_{1}\left(\frac{\lambda|\nabla K(a)|}{M}\right)\left(\frac{1}{\lambda} \frac{\partial \varphi_{a, \lambda}}{\partial a} \frac{\nabla K(a)}{|\nabla K(a)|}-\lambda \frac{\partial \varphi_{a, \lambda}}{\partial \lambda}\right) \\
+\left(1-\psi_{1}\left(\frac{\lambda|\nabla K(a)|}{M}\right)\right)(\operatorname{sign}(-\Delta K(a))) \lambda \frac{\partial \varphi_{a, \lambda}}{\partial \lambda} .
\end{gathered}
$$

We notice that, in the region where $|\nabla K(a)| \geq 2 M / \lambda$, we have that $\psi_{1}(\lambda|\nabla K(a)| / M)=1$, therefore the Claim $(i)$ follows from Proposition 5.7.

Next if $|\nabla K(a)| \leq 2 M / \lambda$ then $a$ is very close to a critical point of $K$ in $\overline{\mathbb{S}_{+}^{n}}$. We claim that this critical point cannot be on the boundary. Indeed, arguing by contradiction, we assume that $a$ is in small neighborhood of a critical point $z \in \partial \mathbb{S}_{+}^{n}$. Since $z$ is a non-degenerate critical point of $K$, we derive that $\lambda d(a, z)$ is bounded which contradicts the fact that $\lambda d\left(a, \partial \mathbb{S}_{+}^{n}\right)$ is very large. Hence our claim follows and $a$ is close to an interior critical point $y$ in $\mathbb{S}_{+}^{n}$.

Next using Proposition 5.7 we derive that

$$
\left\langle-\nabla J_{K}(u), W\right\rangle \geq c \psi_{1}\left(\frac{\lambda|\nabla K(a)|}{M}\right)\left(\frac{|\nabla K(a)|}{\lambda}+\frac{1}{\lambda^{2}}\right)+\left(1-\psi_{1}\left(\frac{\lambda|\nabla K(a)|}{M}\right)\right) \frac{c}{\lambda^{2}}
$$

which implies Claim $(i)$ in this region.

Hence Claim $(i)$ is proved in the case where $p=1$ and $q=0$.

Concerning ( $i i)$ it follows from (i) using the estimate of $\bar{v}$ in Lemma 2.3. Finally we notice that $\lambda$ increases along the flow lines of the pseudogradient $W$ only in the region where $a$ is close to a critical point $y$ with $\Delta K(y)<0$. Thus the proof of the proposition follows in the case where $p=1$ and $q=0$.

Next we consider the case where $p=0$ and $q=1$, that is the case of a boundary concentration point $a \in \partial \mathbb{S}_{+}^{n}$. In this situation we divide the set $V(1,1,0, \varepsilon)$ into 3 subsets and construct an appropriate vector field in each of these sets. 
(1) Let $V_{1}^{1}:=\left\{u \in V(1,1,0, \varepsilon):\left|1-J_{K}(u)^{\frac{n}{n-2}} \alpha^{\frac{4}{n-2}} K(a)\right| \geq M / \mu\right\}$. In this region, we define

$$
\underline{W}_{1}^{1}:=\operatorname{sign}\left(1-J_{K}(u)^{\frac{n}{n-2}} \alpha^{\frac{4}{n-2}} K(a) \mid\right) \delta_{a, \lambda}
$$

and using Proposition 5.6, Claim ( $i$ ) follows easily (since $M$ is chosen large).

(2) Let $V_{1}^{2}:=\left\{u \in V(1,1,0, \varepsilon):\left|1-J_{K}(u)^{\frac{n}{n-2}} \alpha^{\frac{4}{n-2}} K(a)\right| \leq 2 M / \mu\right.$ and $\left|\nabla K_{1}(a)\right| \geq$ $\eta\}$, where $\eta$ is a small fixed constant. In this region, we define

$$
W_{1}^{2}:=\frac{1}{\eta} W_{a}^{b} \quad \text { where } W_{a}^{b}:=\frac{1}{\lambda} \frac{\partial \delta_{a, \lambda}}{\partial a} \frac{\nabla K_{1}(a)}{\left|\nabla K_{1}(a)\right|} .
$$

Note that, in this region, the parameter $\mu$ is of the same order that $\lambda$. Hence, using Proposition 5.5, the proof of Claim $(i)$ follows.

(3) Let $V_{1}^{3}:=\left\{u \in V(1,1,0, \varepsilon):\left|1-J_{K}(u)^{\frac{n}{n-2}} \alpha^{\frac{4}{n-2}} K(a)\right| \leq 2 M / \mu\right.$ and $\left|\nabla K_{1}(a)\right| \leq$ $2 \eta$ \}. In this region, $a$ is close to a critical point $z$ of $K_{1}$. The pseudogradient will depend on z. We define

$$
\begin{gathered}
\underline{W}_{1}^{3}:=\psi_{1}\left(\lambda\left|\nabla K_{1}(a)\right| / M\right) W_{a}^{b}+\gamma \lambda \frac{\partial \delta_{a, \lambda}}{\partial \lambda} \text { with } \gamma \in\{-1,1\} \text { satisfying } \\
\left\{\begin{array}{l}
\gamma=1 \text { if } \partial K / \partial v(z)>0 \text { or } \partial K / \partial v(z)=0 \text { and } \Delta K(z)<0, \\
\gamma=-1 \text { if } \partial K / \partial v(z)<0 \text { or } \partial K / \partial v(z)=0 \text { and } \Delta K(z)>0 .
\end{array}\right.
\end{gathered}
$$

Using Propositions 5.4 and 5.5, it holds

$$
\begin{aligned}
\left\langle-\nabla J_{K}(u), \underline{W}_{1}^{3}\right\rangle \geq & c \psi_{1}\left(\frac{\lambda\left|\nabla K_{1}(a)\right|}{M}\right)\left(\frac{\left|\nabla K_{1}(a)\right|}{\lambda}+\frac{1}{\lambda^{2}}\right) \\
& +\gamma\left(\frac{c_{3}}{\lambda} \frac{\partial K}{\partial v}(a)-c \frac{\Delta K(a)}{\lambda^{2}}+O\left(\frac{1}{\lambda^{3}}\right)\right) .
\end{aligned}
$$

Observe that, if $\partial K / \partial v(z) \neq 0$, it follows that $\gamma \partial K / \partial v(a) \geq c>0$ and therefore Claim (i) follows easily. In the other case, that is $\partial K / \partial v(z)=0$, we need to make use of the assumption (H3). Indeed,

- if $(i)$ of (H3) holds, it follows that $\gamma \partial K / \partial v(a)=|\partial K / \partial v(a)|$ and $-\gamma \Delta K(a) \geq c>0$. Therefore, if $\lambda\left|\nabla K_{1}(a)\right| \geq 2 M$, in the lower bound of (14) will appear $\left|\nabla K_{1}(a)\right| / \lambda+$ $|\partial K / \partial \nu(a)| / \lambda+1 / \lambda^{2}$ which is larger than $c / \mu$. Hence, Claim $(i)$ follows in this case. However, if $\lambda\left|\nabla K_{1}(a)\right| \leq 2 M$, it follows that $|\nabla K(a)| \leq c M / \lambda$ (since we assumed that $z$ is a non degenerate critical point). Therefore $1 / \lambda^{2} \geq c\left(1 / \lambda^{2}+|\nabla K(a)| / \lambda\right)=c / \mu$. Thus Claim $(i)$ follows in this case.

- Next we consider the case where ( $i i)$ of (H3) holds. Recall that $z$ is a non degenerate critical point of $K_{1}$, thus it follows that there exists $r_{1}>0$ such that $\left|\nabla K_{1}(a)\right| \geq \underline{c d}(a, z)$ for each $a \in B\left(z, r_{1}\right)$. Let $\varrho_{1}>0$ (satisfying $\varrho_{1} \max (M, 1 / \underline{c}$ ) is very small), using (ii) of (H3), there exists $r_{2}>0$ (with $\left.r_{2} \leq r_{1}\right)$ such that $|\partial K / \partial v(a)| \leq \varrho_{1} d(a, z)$ for each $a \in B\left(z, r_{2}\right)$. Hence, in $B\left(z, r_{2}\right),|\partial K / \partial v(a)|=o\left(\left|\nabla K_{1}(a)\right|\right.$ ) (since $\varrho_{1}$ is chosen so that $\varrho_{1} / \underline{c}$ is small) and therefore $\left|\nabla K_{1}(a)\right|=|\nabla K(a)|(1+o(1))$. Finally, as before, if $\lambda\left|\nabla K_{1}(a)\right| \geq 2 M$, in the lower bound of (14) will appear $\left|\nabla K_{1}(a)\right| / \lambda$. Furthermore, we have $-\gamma \Delta K(a) \geq c>0$ and $|\partial K / \partial v(a)|=o\left(\left|\nabla K_{1}(a)\right|\right)$ which imply the proof of Claim $(i)$ in this case. In the other case, which is $\lambda\left|\nabla K_{1}(a)\right| \leq 2 M$, it holds: $d(a, z) \leq c M / \lambda$ which implies that $|\partial K / \partial v(a)| \leq \varrho_{1} d(a, z) \leq c \varrho_{1} M / \lambda^{2}=o\left(1 / \lambda^{2}\right)$ (by the chose of $\varrho_{1}$ ). Thus the proof of Claim ( $i$ ) follows from (14).

Finally Claim ( $i i)$ follows from Claim $(i)$ using the estimate of $\bar{v}$ in Lemma 2.3 and Claim (iii) follows immediately from the properties of the constructed vector field. 
We remark that the assumption $(H 2)$ is not used in the construction of the pseudogradient in $V(1,1,0, \varepsilon)$.

\subsection{The case of multiple concentration points}

In the next proposition we address the case where the set of the concentration points contains more than one point. Before stating our result we define for $i=1, \ldots, m$ the scalar quantity $\mu_{i}$ as follows

$$
\mu_{i}^{-1}=\left|\nabla K\left(a_{i}\right)\right| / \lambda_{i}+1 / \lambda_{i}^{2} \text { if } i \leq q ; \quad \mu_{i}=\lambda_{i}^{2} \text { if } i \geq q+1 .
$$

The behavior of such a quantity along the flow lines of the constructed pseudogradient plays crucial role in identifying critical points at infinity.

Proposition 3.2 Assume that $K$ satisfies (H1), (H2) and (H3). A pseudogradient $W$ can be defined so that the following holds: There is a constant $c>0$ independent of $u=$ $\sum_{i=1}^{q} \alpha_{i} \delta_{a_{i}, \lambda_{i}}+\sum_{j=q+1}^{p+q} \alpha_{j} \varphi_{a_{j}, \lambda_{j}} \in V(m, q, p, \varepsilon)$ such that

$$
\begin{aligned}
& \left\langle-\nabla J_{K}(u), W\right\rangle \geq c \sum_{i=1}^{p+q} \frac{1}{\mu_{i}^{2-1 /(n-2)}}+c \sum_{i \leq q}\left|1-J_{K}(u)^{\frac{n}{n-2}} \alpha_{i}^{\frac{4}{n-2}} K\left(a_{i}\right)\right|^{2-\frac{1}{n-2}} \\
& \quad+c \sum_{k \neq r} \varepsilon_{k r}^{\frac{n-1}{n-2}}+c \sum_{i>q}\left(\frac{1}{\left(\lambda_{i} d_{i}\right)^{n-1}}+\left(\frac{\left|\nabla K\left(a_{i}\right)\right|}{\lambda_{i}}\right)^{2-\frac{1}{n-2}}\right)
\end{aligned}
$$

$$
\begin{aligned}
& \left\langle-\nabla J_{K}(u+\bar{v}), W+\frac{\partial \bar{v}}{\partial\left(\alpha_{i}, a_{i}, \lambda_{i}\right)}(W)\right\rangle \geq c \sum_{i=1}^{p+q} \frac{1}{\mu_{i}^{2-1 /(n-2)}}+c \sum_{k \neq r} \varepsilon_{k r}^{\frac{n-1}{n-2}} \\
& \quad+c \sum_{i \leq q}\left|1-J_{K}(u)^{\frac{n}{n-2}} \alpha_{i}^{\frac{4}{n-2}} K\left(a_{i}\right)\right|^{2-\frac{1}{n-2}}+c \sum_{i>q}\left(\frac{1}{\left(\lambda_{i} d_{i}\right)^{n-1}}+\left(\frac{\left|\nabla K\left(a_{i}\right)\right|}{\lambda_{i}}\right)^{2-\frac{1}{n-2}}\right)
\end{aligned}
$$

where $d_{i}:=d\left(a_{i}, \partial \mathbb{S}_{+}^{n}\right)$.

(iii) The vector field $W$ is bounded with the property that along its flow lines the maximum of the $\mu_{i}$ 's increases only if the $(q+p)$-tuple $\left(a_{1}, \ldots, a_{q}, \ldots a_{q+p}\right)$ is close to a collection of different critical points of $K$ or $K_{1}\left(z_{1}, \ldots, z_{q}, y_{q+1}, \ldots y_{q+p}\right)$ with the $y_{i}$ 's are critical points of $K$ in $\mathbb{S}_{+}^{n}$ satistying $\Delta K\left(y_{i}\right)<0$ for each $i \geq q+1$ and the $z_{i}$ 's are critical points of $K_{1}$ such that either $(\partial K / \partial v)\left(z_{i_{k}}\right)>0$ or $\left((\partial K / \partial v)\left(z_{i}\right)=0\right.$ and $\left.\Delta K\left(z_{i}\right)<0\right)$.

The construction of a pseudogradient satisfying $(i),(i i),(i i i)$ is quite involved and requires some preparatory Lemmas and estimates. Its construction depends on the behavior of the leading terms of the $\alpha$-, $a$ - and $\lambda$-component of the gradient in the neighborhood at infinity $V(m, q, p, \varepsilon)$. To perform such a construction we divide the set $V(m, q, p, \varepsilon)$ into four subsets. The first and the second ones correspond to the situation where at least one of the variables $\alpha_{i}$ 's and $a_{i}$ 's is not in its critical position and the $\mu_{i}$ 's are of the same order. In the third one, the $\mu_{i}$ 's are still of the same order but the variables $\alpha_{i}$ 's and $a_{i}$ 's are very close to their critical positions. Finally in the fourth one we address the case where the $\mu_{i}$ 's are not of the same order. 
To define these regions, we introduce the following notation. For $M_{2}$ a large constant we set:

$$
\begin{aligned}
& \Gamma_{\alpha_{k}}:=\frac{\left|1-J_{K}(u)^{\frac{n}{n-2}} \alpha_{k}^{\frac{4}{n-2}} K\left(a_{k}\right)\right|}{M_{2}\left(\sum_{r \neq k} \varepsilon_{k r}+1 / \mu_{k}\right)} ; \quad \Gamma_{a_{i}}^{b}:=\frac{\left|\nabla K_{1}\left(a_{i}\right)\right| / \lambda_{i}}{M_{2} / \lambda_{i}^{2}+\left(1 / M_{2}^{2}\right) \sum_{k \in I} \varepsilon_{i k}} \quad \text { for } i \leq q \\
& \Gamma_{a_{i}}:=\frac{\left|\nabla K\left(a_{i}\right)\right| / \lambda_{i}}{M_{2}\left(\sum_{k \neq i} \varepsilon_{k i}+\left(\lambda_{i} d_{i}\right)^{2-n}+\frac{1}{\lambda_{i}^{2}}\right)} ; \Gamma_{H_{i}}:=H\left(a_{i}, a_{i}\right) / M_{2} \lambda_{i}^{n-4} \quad \text { for } i>q, \\
& \Gamma_{\lambda_{k}}:=\mu_{k} \sum_{j \neq k} \varepsilon_{j k} / M_{2} \quad \text { for } 1 \leq i \leq q+p .
\end{aligned}
$$

To explain the relevance of the above quantities, we state the following Lemma

Lemma 3.3 (1) Let $a_{i}$ be an interior point satisfying $\Gamma_{\lambda_{i}}+\Gamma_{a_{i}}+\Gamma_{H_{i}} \leq 8$. Then $a_{i}$ is close to a interior critical point y of $K$ in $\mathbb{S}_{+}^{n}$.

(2) If $a_{i}, a_{j}$ are interior points satisfying that $\Gamma_{\lambda_{k}}+\Gamma_{a_{k}}+\Gamma_{H_{k}} \leq 8$ for $k=i, j$ and if their corresponding concentration rates $\lambda_{i}$ and $\lambda_{j}$ are of the same order. Then $a_{i}$ and $a_{j}$ cannot be close to the same critical point.

Proof Since $i$ satisfies: $\Gamma_{H_{i}}+\Gamma_{a_{i}}+\Gamma_{\lambda_{i}} \leq 8$, this implies that $\left|\nabla K\left(a_{i}\right)\right| \leq C / \lambda_{i}$ and therefore $a_{i}$ is close to a critical point of $K$. We need to exclude the case where this critical point lies on the boundary. In fact, assuming that it is the case, i.e. $a_{i}$ is close to $z \in \partial \mathbb{S}_{+}^{n}$. Then it follows from $(H 1)$, that $\lambda_{i} d\left(a_{i}, z\right)$ is bounded, which is not allowed. Therefore, each concentration point $a_{i}$ is close to a critical point $y_{j_{i}} \in \mathbb{S}_{+}^{n}$ and the first assertion is proved.

Concerning the second one, assume that two different points $a_{i}$ and $a_{j}$ are near the same critical point $y$. Then we have from the first assertion: $\lambda_{k} d\left(a_{k}, y\right)$ is bounded for $k=i, j$. Since $\lambda_{i}$ and $\lambda_{j}$ are assumed to be of the same order, it follows that $\lambda_{k} d\left(a_{i}, a_{j}\right)$ is bounded, which contradicts the smallness of $\varepsilon_{i j}$.

\subsubsection{Construction of some local pseudogradients}

In this subsection we construct some local pseudogradients in some parts of the neighborhood at infinity. These vector fields will be glued together to obtain a global pseudogradient satisfying the properties required in Proposition 3.2.

For $M_{0}$ a large number we define the following subsets of $V(m, q, p, \varepsilon)$

$$
\begin{aligned}
V_{1}\left(M_{0}\right):= & \left\{u: \mu_{\max } \leq 2 M_{0} \mu_{\min }\right\} \cap\left\{u: \exists i>q: \Gamma_{H_{i}}+\Gamma_{a_{i}}+\Gamma_{\lambda_{i}} \geq 6\right\}, \\
V_{2}\left(M_{0}\right):= & \left\{u: \mu_{\max } \leq 2 M_{0} \mu_{\min }\right\} \cap\left\{u: \forall i>q: \Gamma_{H_{i}}+\Gamma_{a_{i}}+\Gamma_{\lambda_{i}} \leq 8\right\} \cap\left(\left\{u: \exists i \leq q: \Gamma_{\alpha_{i}}+\Gamma_{\lambda_{i}} \geq 4\right\}\right. \\
& \left.\cup\left\{u: \exists i \leq q: d\left(a_{i}, \mathcal{K}^{b}\right) \geq \eta\right\}\right) \quad \text { where } \mathcal{K}^{b}:=\left\{z \in \partial \mathbb{S}_{+}^{n}: \nabla K_{1}(z)=0\right\}, \\
V_{3}\left(M_{0}\right):= & \left\{u: \mu_{\max } \leq 2 M_{0} \mu_{\min }\right\} \cap\left\{u: \forall i>q: \Gamma_{H_{i}}+\Gamma_{a_{i}}+\Gamma_{\lambda_{i}} \leq 8\right\} \cap\left\{u: \forall i \leq q: \Gamma_{\alpha_{i}}+\Gamma_{\lambda_{i}} \leq 6\right\} \\
& \cap\left\{u: \forall i \leq q: d\left(a_{i}, \mathcal{K}^{b}\right) \leq 2 \eta\right\}, \\
V_{4}\left(M_{0}\right):= & \left\{u: \mu_{\max }>M_{0} \mu_{\min }\right\},
\end{aligned}
$$

where $\mu_{\max }:=\max _{j} \mu_{j}$ and $\mu_{\min }:=\min _{j} \mu_{j}$.

Before defining a pseudogradient in each subset, we single out some of their properties that will be used in the construction of the local pseudogradients. 
Remark 3.4 (1) In $V_{k}\left(M_{0}\right)$, for $k \leq 3$, the variables $\mu_{i}$ 's are of the same order. Thus, using Lemma 5.2, we derive that, for each $i \neq j \leq q$, it holds

$$
-\lambda_{i} \frac{\partial \varepsilon_{i j}}{\partial \lambda_{i}} \geq c \varepsilon_{i j}
$$

Furthermore, for $i \neq j>q$, we deduce that $\lambda_{i}$ and $\lambda_{j}$ are of the same order and therefore (17) holds true. Now, for $i>q$ and $j \leq q$, we have $\lambda_{i} d_{i}$ is very large which implies that $\lambda_{i} d\left(a_{i}, a_{j}\right)$ is also very large and therefore (17) holds for these indices.

(2) In $V_{k}\left(M_{0}\right), k=2,3$, for each $i>q$, the concentration point $a_{i}$ is close to a critical point $y_{j_{i}} \in \mathbb{S}_{+}^{n}$ and two different points $a_{i}$ and $a_{j}$ cannot be near the same critical point $y$ (see Lemma 3.3).

(3) In $V_{3}\left(M_{0}\right)$, for each $i \leq q, a_{i}$ is close to a critical point $z_{j_{i}}$ of $K_{1}$ in $\partial \mathbb{S}_{+}^{n}$.

We start our construction by defining a pseudogradient in $V_{1}\left(M_{0}\right)$.

Lemma 3.5 There exists a bounded pseudogradient $W_{1}$ so that the following holds: There is a constant $c>0$ independent of $u=\sum_{i=1}^{q} \alpha_{i} \delta_{i}+\sum_{i=q+1}^{p+q} \alpha_{i} \varphi_{i} \in V_{1}\left(M_{0}\right)$ such that

$$
\begin{aligned}
& \left\langle-\nabla J_{K}(u), W_{1}\right\rangle \geq \sum_{i=1}^{q+p} \frac{c}{\mu_{i}}+c \sum_{i=1}^{q}\left|1-J_{K}(u)^{\frac{n}{n-2}} \alpha_{i}^{\frac{4}{n-2}} K\left(a_{i}\right)\right| \\
& \quad+c \sum_{k \neq r} \varepsilon_{k r}+\sum_{i=q+1}^{q+p} \frac{\left|\nabla K\left(a_{i}\right)\right|}{\lambda_{i}} .
\end{aligned}
$$

Furthermore, the $\lambda_{i}$ 's are decreasing functions along the flow lines generated by this pseudogradient. In addition, the constant of $1 / \mu_{\max }$ is independent of $M_{0}$ and $M_{2}$.

Proof We start by defining the following vector fields:

$$
\begin{aligned}
W_{\Lambda_{i n}} & :=-\sum_{i>q}\left(\psi_{1}\left(\Gamma_{\lambda_{i}}\right)+\psi_{1}\left(\Gamma_{H_{i}}\right)\right) \lambda_{i} \frac{\partial \varphi_{i}}{\partial \lambda_{i}} \quad \text { and } \quad W_{a}^{i n}:=\sum_{i>q} \psi_{1}\left(\Gamma_{a_{i}}\right) \frac{1}{\lambda_{i}} \frac{\partial \varphi_{i}}{\partial a_{i}} \frac{\nabla K\left(a_{i}\right)}{\left|\nabla K\left(a_{i}\right)\right|} \text { (19) } \\
W_{\Lambda_{b}} & :=-\sum_{i \leq q} \psi_{1}\left(\Gamma_{\lambda_{i}}\right) \lambda_{i} \frac{\partial \delta_{i}}{\partial \lambda_{i}} \quad \text { and } \quad W_{\alpha}:=-\sum_{k \leq q} \psi_{1}\left(\Gamma_{\alpha_{k}}\right) \operatorname{sign}\left(1-J_{K}(u)^{\frac{n}{n-2}} \alpha_{k}^{\frac{4}{n-2}} K\left(a_{k}\right)\right) \delta_{k}
\end{aligned}
$$

where $\psi_{1}$ is a $C^{\infty}$ function defined by $\psi_{1} \in[0,1], \psi_{1}(t)=1$ if $t \geq 2$ and $\psi_{1}(t)=0$ if $t \leq 1$. Observe that, using Propositions 5.4, 5.7, the estimate (17) and the definition of $\psi_{1}$, we derive that

$$
\begin{aligned}
& \left\langle-\nabla J_{K}(u), W_{\Lambda_{i n}}\right\rangle \geq c \sum_{i>q}\left(\psi_{1}\left(\Gamma_{\lambda_{i}}\right)+\psi_{1}\left(\Gamma_{H_{i}}\right)\right)\left(\sum_{j \neq i} \varepsilon_{i j}+\frac{H\left(a_{i}, a_{i}\right)}{\lambda_{i}^{n-2}}+\frac{M_{2}}{2} \frac{1}{\lambda_{i}^{2}}+O\left(R_{1}\right)\right):=\bar{\Gamma}_{\Lambda_{i n}} \\
& \left\langle-\nabla J_{K}(u), W_{\Lambda_{b}}\right\rangle \geq c \sum_{i \leq q} \psi_{1}\left(\Gamma_{\lambda_{i}}\right)\left(\sum_{k \neq i} \varepsilon_{i k}+\frac{M_{2}}{2} \frac{1}{\mu_{i}}+O\left(\sum_{k>q} \varepsilon_{k i}+R_{1}^{b}\right)\right):=\bar{\Gamma}_{\Lambda_{b}} .
\end{aligned}
$$

Moreover using Proposition 5.6, we derive that

$$
\left\langle-\nabla J_{K}(u), W_{\alpha}\right\rangle \geq c \sum_{k \leq q} \psi_{1}\left(\Gamma_{\alpha_{k}}\right)\left(\left|1-J_{K}(u)^{\frac{n}{n-2}} \alpha_{k}^{\frac{4}{n-2}} K\left(a_{k}\right)\right|+\frac{M_{2}}{2}\left(\sum_{r \neq k} \varepsilon_{k r}+\frac{1}{\mu_{k}}\right)\right):=\bar{\Gamma}_{\alpha} .
$$


Such an estimate suggests to move the variable $\alpha_{i}$ 's if $\left|1-J_{K}(u)^{n / n-2} \alpha_{i}^{4 / n-2} K\left(a_{i}\right)\right|$ is very large with respect to $\sum_{r \neq k} \varepsilon_{k r}+1 / \mu_{k}$. Furthermore making use of Propositions 5.6 and 5.7, we derive that

$$
\left\langle-\nabla J_{K}(u), W_{a}^{i n}\right\rangle \geq c \sum_{i>q} \psi_{1}\left(\Gamma_{a_{i}}\right)\left(\frac{\left|\nabla K\left(a_{i}\right)\right|}{\lambda_{i}}+\frac{M_{2}}{2}\left(\sum_{k \neq i} \varepsilon_{k i}+\frac{1}{\left(\lambda_{i} d_{i}\right)^{n-2}}+\frac{1}{\lambda_{i}^{2}}\right)\right):=\bar{\Gamma}_{a}^{i n} .
$$

Nest we define

$$
W_{1}:=W_{\Lambda_{i n}}+W_{a}^{i n}+W_{\alpha}+\left(1 / M_{2}\right) W_{\Lambda_{b}} .
$$

Using the previous estimates, we obtain

$$
\begin{aligned}
& \left\langle-\nabla J_{K}(u), W_{1}\right\rangle \geq \bar{\Gamma}_{\Lambda_{i n}}+\bar{\Gamma}_{a}^{i n}+\bar{\Gamma}_{\alpha}+\left(1 / M_{2}\right) \bar{\Gamma}_{\Lambda_{b}} \\
& \geq c \sum_{i>q}\left(\psi_{1}\left(\Gamma_{\lambda_{i}}\right)+\psi_{1}\left(\Gamma_{H_{i}}\right)+\psi_{1}\left(\Gamma_{a_{i}}\right)\right)\left(\sum_{j \neq i} \varepsilon_{i j}+\frac{H\left(a_{i}, a_{i}\right)}{\lambda_{i}^{n-2}}+\frac{M_{2}}{2} \frac{1}{\lambda_{i}^{2}}\right) \\
& +c \sum_{i>q} \psi_{1}\left(\Gamma_{a_{i}}\right) \frac{\left|\nabla K\left(a_{i}\right)\right|}{\lambda_{i}}+\bar{\Gamma}_{\alpha}+\left(1 / M_{2}\right) \bar{\Gamma}_{\Lambda_{b}}+O\left(R_{1}\right) .
\end{aligned}
$$

Regarding the above estimate, we point that we need to take care of the interaction term $O\left(\varepsilon_{k i}\right)$ contained in the expression $\bar{\Gamma}_{\Lambda_{b}}$. To that aim, we observe that, if $\Gamma_{H_{k}}+\Gamma_{a_{k}}+\Gamma_{\lambda_{k}} \geq$ 6 , then the $\varepsilon_{k i}$ appears in the lower bound in (24) and therefore we are able to remove the $\left(1 / M_{2}\right) \varepsilon_{k i}$ by taking $M_{2}$ large. But, if $\Gamma_{H_{k}}+\Gamma_{a_{k}}+\Gamma_{\lambda_{k}} \leq 6$, it follows that (see the second assertion of Remark 3.4) $a_{k}$ is close to a critical point $y$ of $K$ and therefore we get $\varepsilon_{k i}=O\left(1 / \lambda_{k}^{n-2}+1 / \lambda_{i}^{n-2}\right)$ which is small with respect to our lower bound.

Since we are in $V_{1}\left(M_{0}\right)$, there exists at least one index $i>q$ such that $\psi_{1}\left(\Gamma_{\lambda_{i}}\right)+\psi_{1}\left(\Gamma_{H_{i}}\right)+$ $\psi_{1}\left(\Gamma_{a_{i}}\right) \geq 1$. This implies that $1 / \lambda_{i}^{2}=1 / \mu_{i}$ appears in the lower bound of (24). Since all the $\mu_{j}$ 's are of the same order, we are able to make appear all the $1 / \mu_{j}$ 's in this lower bound and Lemma 3.5 follows.

In the next lemma we construct a pseudogradient in the set $V_{2}\left(M_{0}\right)$. Namely we prove:

Lemma 3.6 There exists a bounded pseudogradient $W_{2}$ such that the following holds: There is a constant $c>0$ independent of $u=\sum_{i=1}^{q} \alpha_{i} \delta_{i}+\sum_{i=q+1}^{p+q} \alpha_{i} \varphi_{i} \in V_{2}\left(M_{0}\right)$ such that the statement of Lemma 3.5 holds true with $W_{2}$ instead of $W_{1}$.

Proof First, recall that (see Remark 3.4), in $V_{2}\left(M_{0}\right)$, each interior concentration point $a_{k}$ is close to a critical point of $K$ in $\mathbb{S}_{+}^{n}$ and that two interior concentration points $a_{i}$ and $a_{k}$ cannot be close to the same critical point which implies that $d\left(a_{i}, a_{k}\right) \geq c>0$ and $\varepsilon_{i k}=O\left(1 /\left(\lambda_{k} \lambda_{i}\right)^{(n-2) / 2}\right)$.

Recalling that $\mathcal{K}^{b}:=\left\{z \in \partial \mathbb{S}_{+}^{n}: \nabla K_{1}(z)=0\right\}$ we define the following pseudogradient:

$$
W_{2}:=W_{\alpha}+W_{\Lambda_{b}}+\sum_{i \in D_{1}} \frac{1}{\lambda_{i}} \frac{\partial \delta_{i}}{\partial a_{i}} \frac{\nabla K_{1}\left(a_{i}\right)}{\left|\nabla K_{1}\left(a_{i}\right)\right|} \quad \text { where } D_{1}:=\left\{i \leq q: d\left(a_{i}, \mathcal{K}^{b}\right) \geq \eta\right\} .
$$

Using (22), (23) and Proposition 5.5, we get

$$
\left\langle-\nabla J_{K}(u), W_{2}\right\rangle \geq \bar{\Gamma}_{\alpha}+\bar{\Gamma}_{\Lambda_{b}}+\sum_{i \in D_{1}} \frac{c}{\lambda_{i}}+O\left(\sum_{k \leq q}\left(\frac{1}{\lambda_{i}}\left|\frac{\partial \varepsilon_{k i}}{\partial a_{i}}\right|+\lambda_{k} d\left(a_{k}, a_{i}\right) \varepsilon_{k i}^{\frac{n+1}{n-2}}\right)+R_{1}^{b}+\sum_{k>q} \varepsilon_{k i}\right) .
$$


First, taking $i \in D_{1}$, for $k \leq q$, two cases may occur: $(i)$ either $d\left(a_{i}, a_{k}\right) \leq \eta / 2$, and in this case we get that $\left|\nabla K\left(a_{k}\right)\right| \geq c$ and therefore $\mu_{k}$ and $\lambda_{k}$ are of the same order. Thus $\lambda_{i}$ and $\lambda_{k}$ are of the same order. ( $\left.i i\right)$ or $d\left(a_{i}, a_{k}\right) \geq \eta / 2$. In the two cases, we deduce that

$$
\begin{aligned}
& \varepsilon_{k i}=\frac{1+o(1)}{\left(\lambda_{i} \lambda_{k} d\left(a_{i}, a_{k}\right)^{2}\right)^{\frac{n-2}{2}}} ; \quad \lambda_{k} d\left(a_{k}, a_{i}\right) \varepsilon_{k i}^{\frac{n+1}{n-2}} \leq \frac{c \varepsilon_{k i}^{\frac{n-1}{n-2}}}{\lambda_{i} \mid d\left(a_{i}, a_{k}\right)} \\
& \quad \text { and } \frac{1}{\lambda_{i}}\left|\frac{\partial \varepsilon_{k i}}{\partial a_{i}}\right| \leq \frac{c \varepsilon_{k i}}{\lambda_{i} d\left(a_{i}, a_{k}\right)}=o\left(\varepsilon_{k i}\right) .
\end{aligned}
$$

Secondly, for $i \in D_{1}$, we have $\left|\nabla K_{1}\left(a_{i}\right)\right| \geq c(\eta)$ and therefore $\lambda_{i}$ and $\mu_{i}$ are of the same order. Since all the $\mu_{j}$ 's are assumed to be of the same order, we are able to make appear all the $1 / \mu_{j}$ 's in the lower bound of (25). Finally, for $j \notin D_{1},(i)$ either $\Gamma_{\alpha_{j}} \geq 2$, in this case, the $\left|1-J_{K}(u)^{n /(n-2)} \alpha_{j}^{4 /(n-2)} K\left(a_{j}\right)\right|+\sum \varepsilon_{k j}$ appears in $\bar{\Gamma}_{\alpha},(i i)$ or $\Gamma_{\alpha_{j}} \leq 2$ and $\Gamma_{\lambda_{j}} \geq 2$, in this case $\sum_{k j} \varepsilon_{k j}$ appears in $\bar{\Gamma}_{\Lambda_{b}}$, (iii) or $\Gamma_{\alpha_{j}}+\Gamma_{\lambda_{j}} \leq 4$, in this case we are able to make appear $\left|1-J_{K}(u)^{n /(n-2)} \alpha_{j}^{4 /(n-2)} K\left(a_{j}\right)\right|+\sum \varepsilon_{k j}$ from $1 / \mu_{j}$. Hence the lemma follows.

Next we consider the third set $V_{3}\left(M_{0}\right)$. We notice that in this subset each concentration point $a_{i}$ is close to some critical point of $K$ or $K_{1}$ and for a critical point $z \in \partial \mathbb{S}_{+}^{n}$ of $K_{1}$ (resp. $y \in \mathbb{S}_{+}^{n}$ of $K$ ), we denote by

$$
B_{z}:=\left\{i \leq q: a_{i} \text { is close to } z\right\} \quad ; \quad B_{y}:=\left\{i>q: a_{i} \text { is close to } y\right\} .
$$

We observe that it follows from Remark 3.4 that $\# B_{y} \leq 1$ for each critical point $y$ in $\mathbb{S}_{+}^{n}$. However, it is possible to have $\# B_{z} \geq 2$ for some critical points $z$ 's in $\partial \mathbb{S}_{+}^{n}$.

Next we divide the set $V_{3}\left(M_{0}\right)$ into four subsets. The first three ones are defined as follows:

$V_{3}^{1}:=\left\{u \in V_{3}\left(M_{0}\right): \exists z\right.$ with $\partial K / \partial v(z)=0$ and $\left.\# B_{z} \geq 2\right\}$,

$V_{3}^{2}:=\left(\left\{u: \exists z\right.\right.$ with $\partial K / \partial v(z)<0$ and $\left.B_{z} \neq \emptyset\right\} \bigcup\left\{u: \exists y\right.$ with $\Delta K>0$ and $\left.B_{y} \neq \emptyset\right\}$

$\bigcup\left\{u: \exists z\right.$ with $\partial K / \partial v(z)=0 ; \Delta K(z)>0$ and $\left.\left.\# B_{z} \neq 0\right\}\right) \bigcap\left(V_{3}\left(M_{0}\right) \backslash V_{3}^{1}\right)$,

$$
V_{3}^{3}:=\left\{u \in V_{3}\left(M_{0}\right): \exists z \text { with } \partial K / \partial v(z)>0 \text { and } \# B_{z} \geq 2\right\} \bigcap\left(V_{3}\left(M_{0}\right) \backslash\left(V_{3}^{1} \cup V_{3}^{2}\right)\right)
$$

where $y$ is an interior critical point of $K$ and $z$ is a critical point of $K_{1}$, and the last one is defined as:

$$
\begin{aligned}
\mathcal{W}:=\left\{u \in V_{3}\left(M_{0}\right): \forall i \leq q, a_{i} \text { is close to } z_{i} \in \partial \mathbb{S}_{+}^{n}, \text { with } \# B_{z_{i}}=1 ;(\partial K / \partial v=0 \& \Delta K<0)\right. \\
\quad \text { or } \partial K / \partial v>0\} \bigcap\left\{u \in V_{3}\left(M_{0}\right), \forall j>q, a_{j} \text { is close to } y_{j} \in \mathbb{S}_{+}^{n},\right. \\
\left.\quad \text { with } \# B_{y_{j}}=1 \text { and } \Delta K\left(y_{j}\right)<0\right\} .
\end{aligned}
$$

In the next lemma we construct a pseudogradient in the first subset. Namely we prove the following lemma:

Lemma 3.7 There exists a bounded pseudogradient $W_{3}^{1}$ such that the following holds: There is a constant $c>0$ independent of $u=\sum_{i=1}^{q} \alpha_{i} \delta_{i}+\sum_{i=q+1}^{p+q} \alpha_{i} \varphi_{i} \in V_{3}^{1}$ such that the statement of Lemma 3.5 holds true with $W_{3}^{1}$ instead of $W_{1}$.

Proof Let $z$ be such that $\partial K / \partial v(z)=0$ and $\# B_{z} \geq 2$. Firstly, we claim that:

$$
\text { There exists } k \in B_{z} \text { such that: } \frac{\left|\nabla K_{1}\left(a_{k}\right)\right|}{\lambda_{k}} \geq \frac{M_{2}}{\lambda_{k}^{2}}+\frac{1}{M_{2}^{2}} \sum_{j \neq k} \varepsilon_{j k} \text {. }
$$


Indeed arguing by contradiction, we assume that this claim does not hold. Thus, since $z$ is a non-degenerate critical point of $K_{1}$, we obtain, for each $k \in B_{z}$,

$$
\begin{aligned}
c \frac{d\left(a_{k}, z\right)}{\lambda_{k}} & \leq \frac{\left|\nabla K_{1}\left(a_{k}\right)\right|}{\lambda_{k}} \leq \frac{M_{2}}{\lambda_{k}^{2}}+\frac{1}{M_{2}^{2}} \sum_{j \neq k} \varepsilon_{j k} \\
& \leq \frac{M_{2}}{\lambda_{k}^{2}}+\frac{c}{M_{2}} \frac{1}{\mu_{k}} \leq \frac{c}{M_{2}} \frac{\left|\nabla K\left(a_{k}\right)\right|}{\lambda_{k}}+c \frac{M_{2}}{\lambda_{k}^{2}} \leq c \frac{d\left(a_{k}, z\right)}{M_{2} \lambda_{k}}+c \frac{M_{2}}{\lambda_{k}^{2}}
\end{aligned}
$$

which implies that $\lambda_{k} d\left(a_{k}, z\right)$ is bounded. In addition, from the definition of $\mu_{k}$, we get

$$
\frac{1}{\lambda_{k}^{2}} \leq \frac{1}{\mu_{k}}:=\frac{\left|\nabla K\left(a_{k}\right)\right|}{\lambda_{k}}+\frac{1}{\lambda_{k}^{2}} \leq c \frac{\lambda_{k} d\left(a_{k}, z\right)}{\lambda_{k}^{2}}+\frac{1}{\lambda_{k}^{2}} \leq \frac{c}{\lambda_{k}^{2}} .
$$

Thus, $\mu_{k}$ and $\lambda_{k}^{2}$ are of the same order for each $k \in B_{z}$.

Next let $i$ and $j$ be two different indices in $B_{z}$. We deduce that $\lambda_{j}$ and $\lambda_{i}$ are of the same order and $\lambda_{k} d\left(a_{i}, a_{j}\right)$ is bounded for $k=i, j$. These give a contradiction with the fact that $\varepsilon_{i j}$ is small. Hence our claim follows.

Furthermore observe that, for $k$ satisfying (27), it holds that $\lambda_{k} d\left(a_{k}, z\right) \geq c M_{2}$.

Now, in this region, we define the following vector field:

$$
W_{3}^{1}:=\sum_{i \in D_{2}} \frac{1}{\lambda_{i}} \frac{\partial \delta_{i}}{\partial a_{i}} \frac{\nabla K_{1}\left(a_{i}\right)}{\left|\nabla K_{1}\left(a_{i}\right)\right|} \quad \text { where } D_{2}:=\{i \leq q:(27) \text { holds with } k=i\} .
$$

Using Proposition 5.5, we get

$$
\left\langle-\nabla J_{K}(u), W_{3}^{1}\right\rangle \geq c \sum_{i \in D_{2}} \frac{\left|\nabla K_{1}\left(a_{i}\right)\right|}{\lambda_{i}}+O\left(\sum_{k \leq q}\left(\frac{1}{\lambda_{i}}\left|\frac{\partial \varepsilon_{k i}}{\partial a_{i}}\right|+\lambda_{k} d\left(a_{k}, a_{i}\right) \varepsilon_{k i}^{\frac{n+1}{n-2}}\right)+R_{1}^{b}+\sum_{k>q} \varepsilon_{k i}\right) .
$$

Recall that (see Remark 3.4), in $V_{3}\left(M_{0}\right)$, each concentration point $a_{k}$, for $k>q$ is close to a critical point of $K$ in $\mathbb{S}_{+}^{n}$ which implies that $d\left(a_{i}, a_{k}\right) \geq c>0$ for each $i \leq q$. Hence we get $\varepsilon_{i k}=O\left(1 /\left(\lambda_{k} \lambda_{i}\right)^{(n-2) / 2}\right)$.

Moreover for $i \in D_{2}$ and $k \leq q$ with $k \neq i$, two cases may occur: (i) either $\lambda_{k} \leq M_{0}^{2} M_{2}^{2} \lambda_{i}$, and in this case we get

$$
\frac{1}{\lambda_{i}}\left|\frac{\partial \varepsilon_{i k}}{\partial a_{i}}\right|+\lambda_{k} d\left(a_{k}, a_{i}\right) \varepsilon_{k i}^{\frac{n+1}{n-2}} \leq c \lambda_{k} d\left(a_{i}, a_{k}\right) \varepsilon_{i k}^{\frac{n}{n-2}} \leq c M_{0} M_{2} \sqrt{\lambda_{k} \lambda_{i}} d\left(a_{i}, a_{k}\right) \varepsilon_{i k}^{\frac{n}{n-2}} \leq c M_{0} M_{2} \varepsilon_{i k}^{\frac{n-1}{n-2}},
$$

or (ii) $\lambda_{k} \geq M_{0}^{2} M_{2}^{2} \lambda_{i}$. In this case, since $\mu_{k} \leq 2 M_{0} \mu_{i}$ and $z$ is a non-degenerate critical point of $K_{1}$, it follows that

$$
c \frac{d\left(a_{i}, z\right)}{\lambda_{i}} \leq \frac{\left|\nabla K_{1}\left(a_{i}\right)\right|}{\lambda_{i}} \leq \frac{\left|\nabla K\left(a_{i}\right)\right|}{\lambda_{i}}+\left(\frac{1}{\lambda_{i}^{2}}-2 \frac{M_{0}}{\lambda_{k}^{2}}\right) \leq 2 M_{0} \frac{\left|\nabla K\left(a_{k}\right)\right|}{\lambda_{k}} \leq c M_{0} \frac{d\left(a_{k}, z\right)}{\lambda_{k}}
$$

which implies that $d\left(a_{i}, z\right) / d\left(a_{k}, z\right) \leq c M_{0} \lambda_{i} / \lambda_{k} \leq c /\left(M_{0} M_{2}^{2}\right)$. Thus we deduce that $d\left(a_{i}, a_{k}\right) \geq c M_{0} M_{2}^{2} d\left(a_{i}, z\right)$. Therefore we obtain

$\frac{1}{\lambda_{i}}\left|\frac{\partial \varepsilon_{i k}}{\partial a_{i}}\right|+\lambda_{k} d\left(a_{k}, a_{i}\right) \varepsilon_{k i}^{\frac{n+1}{n-2}} \leq c \lambda_{k} d\left(a_{i}, a_{k}\right) \varepsilon_{i k}^{\frac{n}{n-2}} \leq \frac{c}{\lambda_{i} d\left(a_{i}, a_{k}\right)} \varepsilon_{i k} \leq \frac{1}{M_{0} M_{2}^{2}} \frac{c}{\lambda_{i} d\left(a_{i}, z\right)} \varepsilon_{i k} \leq \frac{c}{M_{2}^{3} M_{0}} \varepsilon_{i k}$

where we have used the fact that $\lambda_{i} d\left(a_{i}, z\right) \geq c M_{2}$. Thus (28) becomes

$$
\left\langle-\nabla J_{K}(u), W_{3}^{1}\right\rangle \geq c \sum_{i \in D_{2}} \frac{\left|\nabla K_{1}\left(a_{i}\right)\right|}{\lambda_{i}}+\frac{M_{2}}{\lambda_{i}^{2}}+\frac{1}{M_{2}^{2}} \sum_{j \neq i} \varepsilon_{i j}+O\left(R_{1}^{b}+\sum \frac{1}{\lambda_{j}^{n-2}}\right) .
$$


Finally, we notice that $\left|\nabla K\left(a_{i}\right)\right| \leq c d\left(a_{i}, z\right) \leq c\left|\nabla K_{1}\left(a_{i}\right)\right| \leq c\left|\nabla K\left(a_{i}\right)\right|$. Thus, in (29), we can make appear $1 / \mu_{i}$ for $i \in D_{2}$ and therefore all the $1 / \mu_{j}$ 's (since there are of the same order) and the proof follows as the proof of the previous lemmas.

Lemma 3.8 There exists a bounded pseudogradient $W_{3}^{2}$ such that the following holds: There is a constant $c>0$ independent of $u=\sum_{i=1}^{q} \alpha_{i} \delta_{i}+\sum_{i=q+1}^{p+q} \alpha_{i} \varphi_{i} \in V_{3}^{2}$ such that the statement of Lemma 3.5 holds true with $W_{3}^{2}$ instead of $W_{1}$.

Proof Let $D_{1}:=\cup_{y: \Delta K(y)>0} B_{y}, D_{2}:=\cup_{z: \partial K / \partial v(z)<0} B_{z}$ and $D_{3}:=\cup_{z: \partial K / \partial v(z)=0 ; \Delta K(z)>0} B_{z}$. We divide this region into two subsets:

1st subset: If $D_{1} \cup D_{2} \neq \emptyset$. In this case, we define

$$
W_{3}^{21}:=-\sum_{i \in D_{1} \cup D_{2}} \lambda_{i} \frac{\partial \varphi_{i}}{\partial \lambda_{i}} .
$$

By using the first assertion of Remark 3.4 and Propositions 5.4 and 5.7, it follows that

$$
\left\langle-\nabla J_{K}(u), W_{3}^{21}\right\rangle \geq c \sum_{i \in D_{1} \cup D_{2}}\left(\sum_{j \neq i} \varepsilon_{i j}+\frac{1}{\mu_{i}}+O\left(\sum \frac{1}{\lambda_{j}^{n-2}}+R_{1}^{b}+R_{1}\right)\right) .
$$

Hence, the proof follows.

2nd subset: $D_{3} \neq \varnothing$. Note that, since we are outside of $V_{3}^{1}$, for $i \in B_{z}$ with $\partial K / \partial v(z)=0$, it holds that $B_{z}=\{i\}$, that is $d\left(a_{i}, a_{j}\right) \geq c>0$ for each $j \neq i$. We define

$$
W_{3}^{22}:=\sum_{i \in D_{3}} \psi_{1}\left(\lambda_{i}\left|\nabla K_{1}\left(a_{i}\right)\right| / M\right) \frac{1}{\lambda_{i}} \frac{\partial \delta_{i}}{\partial a_{i}} \frac{\nabla K_{1}\left(a_{i}\right)}{\left|\nabla K_{1}\left(a_{i}\right)\right|}-\lambda_{i} \frac{\partial \delta_{i}}{\partial \lambda_{i}}
$$

where $M$ is a large constant. We point out that $W_{3}^{22}$ is exactly the sum of of the vector fields $W_{1}^{3}$ (defined in (13)) with $\gamma=-1$. Furthermore, the presence of the function $\psi_{1}$ implies that the point $a_{i}$ moves only if $\left|\nabla K_{1}\left(a_{i}\right)\right| \geq M / \lambda_{i}$.

Using Propositions 5.4 and 5.5, we get

$$
\begin{aligned}
\left\langle-\nabla J_{K}(u), W_{3}^{22}\right\rangle \geq & c \sum_{i \in D_{3}} \psi_{1}\left(\lambda_{i}\left|\nabla K_{1}\left(a_{i}\right)\right| / M\right)\left(\frac{\left|\nabla K_{1}\left(a_{i}\right)\right|}{\lambda_{i}}+\frac{1}{\lambda_{i}^{2}}\right) \\
& -\left(\frac{c_{3}}{\lambda_{i}} \frac{\partial K}{\partial v}\left(a_{i}\right)-c \frac{\Delta K\left(a_{i}\right)}{\lambda_{i}^{2}}\right)+O\left(\sum \frac{1}{\lambda_{j}^{n-2}}+R_{1}^{b}\right)
\end{aligned}
$$

which has the same form as (14). Hence, the same computations and arguments hold and the proof of the lemma follows.

\subsubsection{Ruling out collapsing phenomena}

We point that, the main difference between the $\mathbb{S}^{n}$-case (or the case of an interior blow up point for the $\mathbb{S}_{+}^{n}$-case) and the boundary blow up point case relies essentially on the behavior of the leading term in Propositions 5.4 and 5.7 (namely the $\lambda$-term). Indeed when $\partial K / \partial \nu(z) \neq 0$ and $a_{i}$ is close to a boundary critical point $z \in \partial \mathbb{S}_{+}^{n}$, the leading term behaves like $c / \lambda_{i}$, while for the $\mathbb{S}^{n}$-case (or the case of an interior blow up point in the $\mathbb{S}_{+}^{n}$-case), for $a_{i}$ close to an interior critical point $y$ with $\Delta K(y) \neq 0$, this term behaves like $c / \lambda_{i}^{2}$. This difference on the behavior of the leading term plays a crucial role in the nature of the critical point at infinity. Indeed in [1], for $z$ a critical point of $K_{1}$ (which is not local maximum) satisfying 
$\partial K / \partial v(z)>0$, we proved that $z$ is not a simple blow up point in the sense that $B_{z}$ contains more than one concentration point. In the following lemma, we consider the case of a local maximum point of $K_{1}$ satisfying $\partial K / \partial v(z)>0$ and we will prove that $z$ is a simple blow up point. Namely we prove

Lemma 3.9 Let $z$ be a non degenerate local maximum of $K_{1}$ with $\partial K / \partial v(z)>0$. Then $z$ is a simple blow up. More precisely if \# $B_{z}:=\#\left\{a_{i}\right.$; close to $\left.z\right\}:=q_{1} \geq 2$, then $J_{K}$ admits in the set $V\left(q_{1}, q_{1}, 0, \varepsilon\right)$ a compactifying bounded pseudogradient $W\left(z, q_{1}\right)$. Namely there exits a constant $c>0$ independent of $u=\sum_{i=1}^{q_{1}} \alpha_{i} \delta_{i}$ such that

$$
\left\langle-\nabla J_{K}(u), W\left(z, q_{1}\right)\right\rangle \geq c \sum_{i \leq q_{1}}\left(\frac{1}{\lambda_{i}^{2-1 /(n-2)}}+\left|1-J_{K}(u)^{\frac{n}{n-2}} \alpha_{i}^{\frac{4}{n-2}} K\left(a_{i}\right)\right|^{2-\frac{1}{n-2}}\right)+c \sum_{k \neq r} \varepsilon_{k r}^{\frac{n-1}{n-2}} .
$$

Furthermore, the concentration rates $\lambda_{i}$ 's do not increase along the flow lines generated by this pseudogradient.

For the proof of Lemma 3.9, we make use of the following technical results.

Lemma 3.10 Let $a_{i}, a_{j} \in \partial \mathbb{S}_{+}^{n}$ be concentration points such that the corresponding rates $\lambda_{i}$ and $\lambda_{j}$ are of the same order and $d\left(a_{k}, b\right) \rightarrow 0$ for $k=i, j$ for some point $b \in \partial \mathbb{S}_{+}^{n}$. Then we have

$$
e_{i j}:=\frac{\partial \varepsilon_{i j}}{\partial a_{i}}\left(b-\left\langle a_{i}, b\right\rangle a_{i}\right)+\frac{\partial \varepsilon_{i j}}{\partial a_{j}}\left(b-\left\langle a_{j}, b\right\rangle a_{j}\right) \geq c \varepsilon_{i j} .
$$

Proof Easy computation implies that

$$
\frac{\partial \varepsilon_{i j}}{\partial a_{i}}=(n-2) \lambda_{i} \lambda_{j}\left(a_{j}-a_{i}\right) \varepsilon_{i j}^{n /(n-2)} .
$$

Thus we get

$$
\begin{aligned}
e_{i j} & =(n-2) \lambda_{i} \lambda_{j} \varepsilon_{i j}^{n /(n-2)}\left(\left\langle a_{j}-a_{i}, b-\left\langle a_{i}, b\right\rangle a_{i}\right\rangle+\left\langle a_{i}-a_{j}, b-\left\langle a_{j}, b\right\rangle a_{j}\right\rangle\right) \\
& =(n-2) \lambda_{i} \lambda_{j} \varepsilon_{i j}^{n /(n-2)}\left\langle a_{j}+a_{i}, b\right\rangle\left(1-\left\langle a_{i}, a_{j}\right\rangle\right) \\
& =(n-2) \lambda_{i} \lambda_{j} \varepsilon_{i j}^{n /(n-2)}\left|a_{i}-a_{j}\right|^{2}(1+o(1)) \geq c \varepsilon_{i j} .
\end{aligned}
$$

where $\left|a_{i}-a_{j}\right|$ is the euclidian norm of $a_{i}-a_{j}$ seen as a vector in $\mathbb{R}^{n+1}$.

Lemma 3.11 Let $a, h \in \partial \mathbb{S}_{+}^{n}$ be close to a non degenerate local maximum $z$ of $K_{1}$. Then it holds that

$$
\frac{1}{K_{1}(a)^{n / 2}} \nabla K_{1}(a)(h-\langle a, h\rangle a) \geq-\frac{1}{K_{1}(h)^{n / 2}} \nabla K_{1}(h)(a-\langle a, h\rangle h)+c|a-h|^{2} .
$$

Proof Let

$$
\beta(t):=\frac{h+t(a-h)}{|h+t(a-h)|} \quad, \quad g(t):=\frac{2 /(n-2)}{K_{1}(\beta(t))^{(n-2) / 2}} \quad \text { for } t \in[0,1] .
$$

It is easy to get that

$$
\beta^{\prime}(t)=\frac{1}{|h+t(a-h)|}(a-h-\langle\beta(t), a-h\rangle \beta(t)), \quad\langle\beta(t), a-h\rangle=O\left(|a-h|^{2}\right),
$$


and therefore it holds that $\left|\beta^{\prime}(t)\right|=|a-h|(1+o(1))$ uniformly in $t \in[0,1]$. Furthermore, easy computations imply that $\left|\beta^{\prime \prime}(t)\right|=O\left(|a-h|^{2}\right)$ uniformly in $t \in[0,1]$. In another hand, we have

$$
g^{\prime}(t)=\frac{-1}{K_{1}(\beta(t))^{n / 2}} \nabla K_{1}(\beta(t))\left(\beta^{\prime}(t)\right)
$$

and, since $a$ and $h$ are close to a non degenerate maximum critical point $z$ of $K_{1}$, we derive that

$$
\begin{aligned}
g^{\prime \prime}(t) & =o\left(\left|\beta^{\prime}(t)\right|^{2}\right)-\frac{1}{K_{1}(\beta(t))^{n / 2}} D^{2} K_{1}(\beta(t))\left(\beta^{\prime}(t), \beta^{\prime}(t)\right)+o\left(\left|\beta^{\prime \prime}(t)\right|\right) \\
& \left.\geq c|a-h|^{2} \text { (uniformly in } t \in[0,1]\right) .
\end{aligned}
$$

Now,

$\frac{1}{K_{1}(a)^{n / 2}} \nabla K_{1}(a)(h-\langle a, h\rangle a)+\frac{1}{K_{1}(h)^{n / 2}} \nabla K_{1}(h)(a-\langle a, h\rangle h)=g^{\prime}(1)-g^{\prime}(0)=\int_{0}^{1} g^{\prime \prime}(t) d t$ which implies the lemma.

Proof of Lemma 3.9 For the construction of a suitable vector field satisfying the properties required in Lemma 3.9 as well for later purposes we will use some constants $M_{0}, M_{2}$ and $M_{4}$ which are required to be large and to satisfy

$$
\frac{M_{0}}{M_{4}^{2}} \text { small } \quad, \quad \max \left(\frac{M_{2}}{M_{0}^{1 /(q+p-1)}} ; \frac{M_{2}^{(n-1) /(n-2)}}{M_{0}^{(1 / 2+1 /(n-2)) /(q+p-1)}}\right) \text { small . }
$$

The first requirement is used in (34) and (35) below while the second one is used when studying a remainder term of (42) and the last one is used in (44) in the proof of Lemma 3.14 .

In view of the pseudogradient constructed in Lemmas 3.6 and 3.14, it is enough to construct a pseudogradient satisfying the above estimate in the following set:

$V\left(z, q_{1}, \eta, \varepsilon, M_{0}\right):=\left\{u \in V\left(q_{1}, q_{1}, 0, \varepsilon\right): \lambda_{\max } \leq M_{0} \lambda_{\min } ; d\left(a_{i}, z\right)<\eta ; \Gamma_{\lambda_{i}} \leq 2\right.$ and $\left.\Gamma_{\alpha_{i}} \leq 2 \forall i\right\}$.

Moreover, since the $\lambda_{i}$ 's are of the same order, we have that $\varepsilon_{i j}=(1+o(1)) /\left(\lambda_{i} \lambda_{j}\right.$ $\left.d\left(a_{i}, a_{j}\right)^{2}\right)^{(n-2) / 2}$ and therefore $d\left(a_{i}, a_{j}\right) \geq c / \lambda_{1}^{(n-3) /(n-2)}$ for each $i \neq j$ (since $\Gamma_{\lambda_{i}}$ is bounded). We want to construct a pseudogradient which moves the concentration points $a_{i}$ to their barycenter and prove that along its flow lines the Euler-Lagrange functional decreases. To this aim, let $i$ and $i_{1}$ be such that $d\left(a_{i}, a_{i_{1}}\right):=\min d\left(a_{r}, a_{\ell}\right)$ and define $L_{i}:=\left\{i, i_{1}\right\}$. Next let $M_{4}$ be a large positive constant, for such an index $i$, we define inductively a sequence $L_{i}^{s}$ by setting

$$
\begin{aligned}
& L_{i}^{1}:=\left\{j: \exists \ell \in L_{i} \text { s.t. } d\left(a_{j}, a_{\ell}\right) \leq M_{4} d\left(a_{i}, a_{i_{1}}\right)\right\} \text { and } \\
& L_{i}^{s}:=\left\{j: \exists \ell \in L_{i}^{s-1} \text { s.t. } d\left(a_{j}, a_{\ell}\right) \leq M_{4} \max _{r, t \in L_{i}^{s-1}} d\left(a_{r}, a_{t}\right)\right\} .
\end{aligned}
$$

Observe that, since we have only $q_{1}$ points and $\# L_{i}=2$, then there exists $m \leq q_{1}-1$ such that $L_{i}^{m+1}=L_{i}^{m}$ and we set $L_{i}^{*}:=L_{i}^{m}$ where $m$ is the first index such that $L_{i}^{m+1}=L_{i}^{m}$. We remark that $L_{i} \subset L_{i}^{*}$. Next we want to move the points $a_{j}$ 's, for $j \in L_{i}^{*}$, to their center of mass. For this aim, let $\overline{\mathbf{a}}_{\mathbf{i}}$ be defined as

$$
\overline{\mathbf{a}}_{\mathbf{i}}:=\frac{b_{i}}{\left|b_{i}\right|} \quad \text { where } \quad b_{i} \in \mathbb{R}^{n+1} \text { satisfying } \sum_{j \in L_{i}^{*}}\left(b_{i}-a_{j}\right)=0 .
$$


Note that, it is easy to see that $\overline{\mathbf{a}}_{\mathbf{i}}$ satisfies

$$
\overline{\mathbf{a}}_{\mathbf{i}} \in \partial \mathbb{S}_{+}^{n} \quad \text { and } \quad \sum_{j \in L_{i}^{*}} a_{j}-\left\langle a_{j}, \overline{\mathbf{a}}_{\mathbf{i}}\right\rangle \overline{\mathbf{a}}_{\mathbf{i}}=0 .
$$

Now we define the following vector field:

$$
W_{3}^{i}:=\frac{1}{\lambda_{i} \gamma_{i}} \sum_{j \in L_{i}^{*}} \alpha_{j} \frac{\partial \delta_{j}}{\partial a_{j}}\left(\overline{\mathbf{a}}_{\mathbf{i}}-\left\langle a_{j}, \overline{\mathbf{a}}_{\mathbf{i}}\right\rangle a_{j}\right) \quad \text { where } \quad \gamma_{i}:=\max _{j \in L_{i}^{*}} d\left(a_{i}, a_{j}\right) .
$$

We note that $L_{i}^{*}$ has two important properties:

- If $k, \ell \in L_{i}^{*}$, we have $d\left(a_{k}, a_{\ell}\right) \leq c M_{4}^{m} d\left(a_{i}, a_{i_{1}}\right)$.

- If $k \notin L_{i}^{*}$, then, for each $j \in L_{i}^{*}$, we have $d\left(a_{j}, a_{k}\right) \geq M_{4} \max _{r, \ell \in L_{i}^{*}} d\left(a_{r}, a_{\ell}\right)$. Hence, for $k \notin L_{i}^{*}$ and $j \in L_{i}^{*}$, choosing $M_{0}^{(n-2) / 2} / M_{4}^{n-2}$ small, it follows that for every $\ell \in L_{i}^{*}$, we have that:

$$
\begin{aligned}
& \left|\frac{\partial \varepsilon_{j k}}{\partial a_{j}} \| \overline{\mathbf{a}}_{\mathbf{i}}-\left\langle a_{j}, \overline{\mathbf{a}}_{\mathbf{i}}\right\rangle a_{j}\right| \leq \frac{c d\left(\overline{\mathbf{a}}_{\mathbf{i}}, a_{j}\right)}{\left(\lambda_{j} \lambda_{k}\right)^{\frac{n-2}{2}} d\left(a_{j}, a_{k}\right)^{n-1}} \leq \frac{M_{0}^{(n-2) / 2}}{M_{4}^{n-1}} \frac{c}{\left(\lambda_{j} \lambda_{\ell}\right)^{\frac{n-2}{2}} d\left(a_{j}, a_{\ell}\right)^{n-2}}=o\left(\varepsilon_{j \ell}\right) \\
& \varepsilon_{j k} \leq \frac{c}{\left(\lambda_{j} \lambda_{k}\right)^{(n-2) / 2} d\left(a_{j}, a_{k}\right)^{n-2}} \leq \frac{c M_{0}^{(n-2) / 2}}{M_{4}^{n-2}} \frac{1}{\left(\lambda_{j} \lambda_{\ell}\right)^{(n-2) / 2} d\left(a_{j}, a_{\ell}\right)^{n-2}}=o\left(\varepsilon_{j \ell}\right)
\end{aligned}
$$

(by using (31)). We note that, in this region, we have $\left|1-J_{K}(u)^{\frac{n}{n-2}} \alpha_{j}^{\frac{4}{n-2}} K\left(a_{j}\right)\right| \leq$ $c M_{2} / \lambda_{j}$ for each $j$, hence Proposition 5.5 can be written as:

$$
\begin{aligned}
& \left\langle\nabla J_{K}(u), \alpha_{j} \frac{\partial \delta_{j}}{\partial a_{j}}\right\rangle=\lambda_{j}\left[c_{4}\left(1-J_{K}(u)^{\frac{n}{n-2}} \alpha_{i}^{\frac{4}{n-2}} K\left(a_{i}\right)\right)+J_{K}(u)^{\frac{n}{n-2}} \alpha_{i}^{\frac{4}{n-2}} \frac{c_{5}}{\lambda_{i}} \frac{\partial K}{\partial v}\left(a_{i}\right)\right] e_{n} \\
& -J_{K}(u) c_{2} \sum_{k \neq j} \alpha_{j} \alpha_{k} \frac{\partial \varepsilon_{k j}}{\partial a_{j}}-8 c_{5} J_{K}(u)^{-\frac{n-2}{2}} \frac{\nabla K_{1}\left(a_{j}\right)}{K\left(a_{j}\right)^{n / 2}}+O\left(\frac{1}{\lambda}+\lambda \sum \varepsilon_{k r}^{\frac{n}{n-2}} \ln \left(\varepsilon_{k r}^{-1}\right)\right) .
\end{aligned}
$$

Hence we derive that:

$$
\begin{aligned}
\left\langle-\nabla J_{K}(u),\right. & \left.W_{3}^{i}\right\rangle=\frac{J_{K}(u) c_{2}}{\lambda_{i} \gamma_{i}} \sum_{k \neq j ; j \in L_{i}^{*}} \alpha_{j} \alpha_{k} \frac{\partial \varepsilon_{k j}}{\partial a_{j}}\left(\overline{\mathbf{a}}_{\mathbf{i}}-\left\langle a_{j}, \overline{\mathbf{a}}_{\mathbf{i}}\right\rangle a_{j}\right) \\
+ & \frac{8 c_{5} J_{K}(u)^{(2-n) / 2}}{\lambda_{i} \gamma_{i}} \sum_{j \in L_{i}^{*}} \frac{1}{K\left(a_{j}\right)^{n / 2}} \nabla K_{1}\left(a_{j}\right)\left(\overline{\mathbf{a}}_{\mathbf{i}}-\left\langle a_{j}, \overline{\mathbf{a}}_{\mathbf{i}}\right\rangle a_{j}\right) \\
+ & O\left(\frac{1}{\lambda^{2}}+\sum \varepsilon_{k r}^{\frac{n}{n-2}} \ln \left(\varepsilon_{k r}^{-1}\right)\right) .
\end{aligned}
$$

Next we notice that, using Lemma 3.10, il holds

$$
\frac{\partial \varepsilon_{k j}}{\partial a_{j}}\left(\overline{\mathbf{a}}_{\mathbf{i}}-\left\langle a_{j}, \overline{\mathbf{a}}_{\mathbf{i}}\right\rangle a_{j}\right)+\frac{\partial \varepsilon_{k j}}{\partial a_{k}}\left(\overline{\mathbf{a}}_{\mathbf{i}}-\left\langle a_{k}, \overline{\mathbf{a}}_{\mathbf{i}}\right\rangle a_{k}\right) \geq c \varepsilon_{k j}, \quad \text { for each } k, j \in L_{i}^{*} .
$$


Furthermore, using Lemma 3.11 (with $h=\overline{\mathbf{a}}_{\mathbf{i}}$ ), it holds that

$$
\begin{aligned}
& \sum_{j \in L_{i}^{*}} \frac{1}{K\left(a_{j}\right)^{n / 2}} \nabla_{T} K\left(a_{j}\right)\left(\overline{\mathbf{a}}_{\mathbf{i}}-\left\langle a_{j}, \overline{\mathbf{a}}_{\mathbf{i}}\right\rangle a_{j}\right) \\
& \quad \geq \sum_{j \in L_{i}^{*}} \frac{-1}{K\left(\overline{\mathbf{a}}_{\mathbf{i}}\right)^{n / 2}} \nabla_{T} K\left(\overline{\mathbf{a}}_{\mathbf{i}}\right)\left(a_{j}-\left\langle a_{j}, \overline{\mathbf{a}}_{\mathbf{i}}\right\rangle \overline{\mathbf{a}}_{\mathbf{i}}\right)+c \sum_{j \in L_{i}^{*}}\left|a_{j}-\overline{\mathbf{a}}_{\mathbf{i}}\right|^{2} \\
& \geq c \sum_{j \in L_{i}^{*}}\left|a_{j}-\overline{\mathbf{a}}_{\mathbf{i}}\right|^{2} \quad\left(\text { since } \sum_{j \in L_{i}^{*}} a_{j}-\left\langle a_{j}, \overline{\mathbf{a}}_{\mathbf{i}}\right\rangle \overline{\mathbf{a}}_{\mathbf{i}}=0(\text { see }(33))\right) .
\end{aligned}
$$

Thus we get

$$
\left\langle-\nabla J_{K}(u), W_{3}^{i}\right\rangle \geq c \sum_{k, j \in L_{i}^{*}} \frac{\varepsilon_{k j}}{\lambda_{i} \gamma_{i}}+\sum_{j \in L_{i}^{*}} \frac{d\left(a_{j}, \overline{\mathbf{a}}_{\mathbf{i}}\right)^{2}}{\lambda_{i} \gamma_{i}}+O\left(\sum \varepsilon_{\ell r}^{\frac{n}{n-2}} \ln \varepsilon_{\ell r}^{-1}+\frac{1}{\lambda_{i}^{2}}\right) .
$$

Now, since $\gamma_{i}:=\max _{k, r \in L_{i}^{*}} d\left(a_{k}, a_{r}\right)$ is of the same order of all the $d\left(a_{\ell}, a_{j}\right)$ 's, we derive that $\varepsilon_{k j} / \lambda_{i} \gamma_{i} \geq c \varepsilon_{k j}^{(n-1) /(n-2)}$. Furthermore, $\sum_{j \in L_{i}^{*}} d\left(a_{j}, \overline{\mathbf{a}}_{\mathbf{i}}\right)^{2} \geq c \sum_{j, r \in L_{i}^{*}} d\left(a_{j}, a_{r}\right)^{2}$ and therefore

$$
\sum_{j \in L_{i}^{*}} d\left(a_{j}, \overline{\mathbf{a}}_{\mathbf{i}}\right)^{2} /\left(\lambda_{i} \gamma_{i}\right) \geq \sum_{j, r \in L_{i}^{*}} d\left(a_{j}, a_{r}\right) / \lambda_{i} \geq c / \lambda_{i}^{2-1 /(n-2)} .
$$

Hence, in the lower bound of (39), we are able to make appear $1 / \lambda_{i}^{2-1 /(n-2)}$ and therefore (since all the $\lambda_{j}$ 's are of the same order and $\Gamma_{\alpha_{k}} \leq 4$ for each $k$ ) we are able to make appear

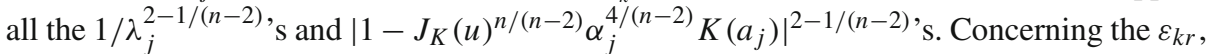
we note that the $\varepsilon_{k j}$ 's which appeared in the lower bound, are only for the indices $k, j \in L_{i}^{*}$. Hence we need to make appear $\varepsilon_{j r}$ for $j \notin L_{i}^{*}$. For this aim, we remark that, for each $j, \ell$, we have $d\left(a_{j}, a_{\ell}\right) \geq d\left(a_{i}, a_{i_{1}}\right)$ (by the definition of $i$ and $\left.i_{1}\right)$, in addition we have that the $\lambda_{k}$ 's are of the same order. Hence we deduce that $\varepsilon_{i i_{1}} \geq c \varepsilon_{j \ell}$. Hence the proof of the lemma follows.

In the next lemma we rule out non simple blow up for a mixed configuration involving local maxima on the boundary and other interior blow up points. Namely we prove:

Lemma 3.12 There exists a bounded pseudogradient $W_{3}^{3}$ such that the following holds: There is a constant $c>0$ independent of $u=\sum_{i=1}^{q} \alpha_{i} \delta_{i}+\sum_{i=q+1}^{p} \alpha_{i} \varphi_{i} \in V_{3}^{3}$ such that

$$
\begin{aligned}
\left\langle-\nabla J_{K}(u), W_{3}^{3}\right. & \geq \sum_{i=1}^{q+p} \frac{c}{\mu_{i}^{\frac{2 n-5}{n-2}}}+c \sum_{i=1}^{q}\left|1-J_{K}(u)^{\frac{n}{n-2}} \alpha_{i}^{\frac{4}{n-2}} K\left(a_{i}\right)\right|^{\frac{2 n-5}{n-2}} \\
& +c \sum_{k \neq r} \varepsilon_{k r}^{\frac{n-1}{n-2}}+c \sum_{i=q+1}^{q+p}\left(\frac{\left|\nabla K\left(a_{i}\right)\right|}{\lambda_{i}}\right)^{\frac{2 n-5}{n-2}}
\end{aligned}
$$

Furthermore, the $\lambda_{i}$ 's do not increase along the flow lines generated by the pseudogradient $W_{3}^{3}$.

Proof Let $z_{1}, \ldots, z_{\ell}$ be the critical points of $K_{1}$ satisfying $\partial K / \partial v\left(z_{j}\right)>0$ and $\# B_{z_{j}} \geq 2$. We decompose $u$ as follows:

$$
u:=\sum_{i=1}^{\ell} u_{i}+u_{\ell+1} \quad \text { where } u_{i}:=\sum_{k \in B_{z_{i}}} \alpha_{k} \delta_{k} \text { and } u_{\ell+1}:=u-\sum_{i=1}^{\ell} u_{i} .
$$


From the second and the third assertions of Remark 3.4, it follows that each concentration point $a_{j}$ of $u_{\ell+1}$ satisfies $\left|a_{j}-a_{k}\right| \geq c$ for each $k \neq j$ and it is close to a critical point of $K_{1}$ with $\partial K / \partial v \geq 0$ or a critical point of $K$ in $\mathbb{S}_{+}^{n}$ with $\Delta K<0$. Furthermore, for $j \in B_{z_{i}}$, we have $\left|a_{j}-a_{k}\right| \geq c$ for each $k \notin B_{z_{i}}$. Hence the mutual interaction between two clusters $B_{z_{i}}$ and $B_{z_{j}}$ for $i \neq j$ is negligible with respect to the other terms. In this situation, we define the following vector field

$$
W_{3}^{3}:=\sum_{i=1}^{\ell} W\left(z_{i}, \# B_{z_{i}}\right)
$$

where $W\left(z_{i}, \# B_{z_{i}}\right)$ is defined in Lemma 3.9. Hence we obtain

$\left\langle-\nabla J_{K}(u), W_{3}^{3}\right\rangle=\sum_{i=1}^{\ell}\left\langle-\nabla J_{K}(u), W\left(z_{i}, \# B_{z_{i}}\right)\right\rangle=\sum_{i=1}^{\ell}\left\langle-\nabla J_{K}\left(u_{i}\right), W\left(z_{i}, \# B_{z_{i}}\right)\right\rangle+\sum_{k \in B_{z_{i}} ; j \notin B_{z_{i}}} O\left(\varepsilon_{k j}\right)$.

We observe that, for $k \in B_{z_{i}}$, we have $\mu_{k}$ and $\lambda_{k}$ are of the same order. Moreover we are in the case where all the $\mu_{j}$ 's are of the same order. Thus, using Lemma 3.9, we are able to make appear all the $1 / \mu_{j}^{2-1 /(n-2)}$, s in the lower bound of (40) (and therefore all the $\left|1-J_{K}(u)^{n /(n-2)} \alpha_{i}^{4 /(n-2)} K\left(a_{i}\right)\right|^{2-1 /(n-2)}$ 's and the $\left(\left|\nabla K\left(a_{i}\right)\right| / \lambda_{i}\right)^{2-1 /(n-2)}$ 's (since the $\Gamma_{\alpha_{k}}$ 's and the $\Gamma_{a_{i}}$ 's are bounded). In addition, for $j \notin B_{z_{i}}$ and $k \in B_{z_{i}}$, we have

$$
\varepsilon_{k j} \leq \frac{c}{\left(\lambda_{j} \lambda_{k}\right)^{(n-2) / 2}} \leq\left\{\begin{array}{l}
o\left(1 / \lambda_{k}^{2}\right) \text { if } n \geq 6 \\
c / \lambda_{k}^{2}+c / \lambda_{j}^{4} \text { if } n=5 .
\end{array}\right.
$$

Therefore, our lemma follows from Lemma 3.9.

Lemma 3.13 There exists a bounded pseudogradient $\mathcal{V}$ satisfying the following estimate:

There is a constant $c>0$ independent of $u=\sum_{i=1}^{q} \alpha_{i} \delta_{i}+\sum_{i=q+1}^{p} \alpha_{i} \varphi_{i} \in \mathcal{W}$ such that (18) holds true with $\mathcal{V}$ instead of $W_{1}$.

Furthermore in the subset of $\mathcal{W}$ such that $\lambda_{i}\left|\nabla K_{1}\left(a_{i}\right)\right|$ is bounded, the $\lambda_{i}$ 's are increasing functions along the flow lines generated by the pseudogradient $\mathcal{V}$.

Proof Let $\psi_{1}$ be a $C^{\infty}$ cut of function defined by $\psi_{1} \in[0,1], \psi_{1}(t)=1$ if $t \geq 2$ and $\psi_{1}(t)=0$ if $t \leq 1$.

We define the following vector field:

$$
\mathcal{V}:=W_{\alpha}+W_{a}^{i n}+W_{a}^{b}+\sum_{i=1}^{p+q} \lambda_{i} \frac{\partial \varphi_{i}}{\partial \lambda_{i}}
$$

where $W_{a}^{b}:=\sum_{i \in I_{b}} \psi_{1}\left(\lambda_{i}\left|\nabla K_{1}\left(a_{i}\right)\right| / M_{2}\right)\left(1 / \lambda_{i}\right)\left(\partial \delta_{i} / \partial a_{i}\right)\left(\nabla K_{1}\left(a_{i}\right) /\left|\nabla K_{1}\left(a_{i}\right)\right|\right)$ and $W_{a}^{i n}$ (resp. $W_{\alpha}$ ) is defined in (19) (resp. (20)).

Observing that in $\mathcal{W}$ we have $\varepsilon_{i j}=O\left(1 / \lambda_{i}^{n-2}+1 / \lambda_{j}^{n-2}\right)$ for each $i \neq j$ and using Propositions 5.4, 5.5, 5.7 the lemma follows.

\subsubsection{Ruling out bubble towers phenomena}

In this subsection we prove any configuration of points of non comparable concentration rates is not critical at infinity. Indeed one can construct in the neighborhood of such points a compactifying pseudogradient. Namely we prove that: 
Lemma 3.14 There exists a bounded pseudogradient $W_{4}$ such that the following holds: There is a constant $c>0$ independent of $u=\sum_{i=1}^{q} \alpha_{i} \delta_{i}+\sum_{i=q+1}^{p+q} \alpha_{i} \varphi_{i} \in V_{4}\left(M_{0}\right)$ such that

$$
\begin{aligned}
\left\langle-\nabla J_{K}(u), W_{4}\right\rangle & \geq c \sum_{i=1}^{q+p} \frac{1}{\mu_{i}^{\frac{n-1}{n-2}}}+c \sum_{i=1}^{q}\left|1-J_{K}(u)^{\frac{n}{n-2}} \alpha_{i}^{\frac{4}{n-2}} K\left(a_{i}\right)\right|^{\frac{n-1}{n-2}} \\
& +c \sum_{k \neq r} \varepsilon_{k r}^{\frac{n-1}{n-2}}+\sum_{i=q+1}^{q+p}\left(\frac{\left|\nabla K\left(a_{i}\right)\right|}{\lambda_{i}}\right)^{\frac{n-1}{n-2}} .
\end{aligned}
$$

Furthermore, $\max \mu_{i}$ deos not increase along the flow lines generated by this pseudogradient.

Proof For $u=\sum_{i=1}^{q} \alpha_{i} \delta_{a_{i}, \lambda_{i}}+\sum_{i=q+1}^{p+q} \alpha_{i} \varphi_{a_{i}, \lambda_{i}}$, we denote

$$
\mathcal{I}_{\text {in }}:=\left\{i=1, \ldots, p+q ; a_{i} \in \mathbb{S}_{+}^{n}\right\} \quad \& \quad \mathcal{I}_{b}:=\left\{i=1, \ldots, p+q ; a_{i} \in \partial \mathbb{S}_{+}^{n}\right\} .
$$

Next we reorder the parameters $\mu_{i}$ 's as: $\mu_{1} \leq \cdots \leq \mu_{p+q}$ and define the following subset of indices:

$$
I:=\{1\} \cup\left\{i \geq 2: \mu_{k} \leq M_{0}^{1 /(p+q-1)} \mu_{k-1} \text { for each } k \leq i\right\} .
$$

Since we are in $V_{4}\left(M_{0}\right)$, we have $\mu_{\max }>M_{0} \mu_{\min }$, it follows that $p+q \notin I$. In this region, we write $u$ as

$$
u:=u_{1}+u_{2} \quad \text { where } u_{1}:=\sum_{i \in I} \alpha_{i} \varphi_{i} \text { and } u_{2}:=u-u_{1} .
$$

Let $k_{0}:=\max I$ (then we have $k_{0}<p+q$ ). It follows that $\mu_{k_{0}} \leq M_{0}^{\left(k_{0}-1\right) /(p+q-1)} \mu_{1}:=$ $\bar{M}_{0} \mu_{1}, \mu_{k_{0}+1} \geq M_{0}^{1 /(p+q-1)} \mu_{k_{0}}$ and therefore $u_{1} \in V_{1}\left(\bar{M}_{0}\right) \cup V_{2}\left(\bar{M}_{0}\right) \cup V_{3}\left(\bar{M}_{0}\right)$.

Furthermore we introduce the following notation

$$
D_{1}^{4}:=\left\{i \in \mathcal{I}_{\text {in }}: \Gamma_{\lambda_{i}}+\Gamma_{a_{i}}+\Gamma_{H_{i}} \geq 6\right\} \quad \& \quad D_{2}^{4}:=\left\{i \in \mathcal{I}_{b}: \Gamma_{\lambda_{i}}+\Gamma_{\alpha_{i}} \geq 4\right\}
$$

and set

$$
i_{0}:=\left\{\begin{array}{ll}
\min D_{1}^{4}, & \text { if } D_{1}^{4} \neq \varnothing \\
p+q+1, & \text { otherwise. }
\end{array} \quad j_{0}:= \begin{cases}\min D_{2}^{4}, & \text { if } D_{2}^{4} \neq \emptyset \\
p+q+1, & \text { otherwise }\end{cases}\right.
$$

Next we define in case $D_{1}^{4} \cup D_{2}^{4} \neq \emptyset$ the following vector fields:

$$
W_{i_{0}}:=-\sum_{i \geq i_{0} ; i \in \mathcal{I}_{i n}} 2^{i} \lambda_{i} \frac{\partial \varphi_{i}}{\partial \lambda_{i}} \text { and } W_{j_{0}}:=-\sum_{j \geq j_{0} ; i \in \mathcal{I}_{b}} 2^{j} \lambda_{j} \frac{\partial \delta_{i}}{\partial \lambda_{i}}
$$

and as in the proof of Lemma 3.5, we define

$$
W_{4}^{0}:=W_{i_{0}}+\left(1 / M_{2}\right) W_{j_{0}}+W_{\alpha}+W_{a}^{i n}
$$

where $W_{a}^{i n}$ (resp. $W_{\alpha}$ ) is defined in (19) (resp. (20)). Following the proof of Lemma 3.5 and using Lemma 5.2, we get

$$
\begin{aligned}
\left\langle-\nabla J_{K}(u), W_{4}^{0}\right\rangle \geq & \bar{\Gamma}_{a}^{i n}+c \sum_{i \geq i_{0} ; i \in \mathcal{I}_{i n}}\left(\sum_{\ell \neq i} \varepsilon_{i \ell}+\frac{1}{\left(\lambda_{i} d_{i}\right)^{n-2}}+O\left(R_{1}\right)\right)+\frac{c}{\mu_{i_{0}}} \\
& +\bar{\Gamma}_{\alpha}+\frac{c}{M_{2}} \sum_{j \geq j_{0} ; j \in \mathcal{I}_{b}}\left(\sum_{\ell \neq j} \varepsilon_{j \ell}+O\left(R_{1}^{b}++c \frac{c}{\mu_{j_{0}}}+\sum_{\ell \in \mathcal{I}_{i n}} \varepsilon_{j \ell}\right)\right):=\bar{\Gamma}_{4} .
\end{aligned}
$$


Observe that, concerning the last term, for $\ell \in \mathcal{I}_{i n}$, $(i)$ either $\ell \geq i_{0}$, then the $\varepsilon_{j \ell}$ exists in the second term of this formula and one takes $M_{2}$ large to absorb the last term, or ( $i$ i $) \ell<i_{0}$ and in this case by Lemma 3.3, the concentration point $a_{\ell}$ is close to a critical point $y$ of $K$ in $\mathbb{S}_{+}^{n}$ and then $\varepsilon_{j \ell} \leq c\left(1 / \lambda_{j}^{n-2}+1 / \lambda_{\ell}^{n-2}\right)$. Hence, we can in either case absorb the last term.

Furthermore we notice that if $D_{1}^{4} \cup D_{2}^{4} \neq \varnothing$ and if $i_{0} \in I$ or if $j_{0} \in I$ then we can include all the indices in $I$ in the lower bound of (41). Otherwise to make appear the terms corresponding to these indices we argue as follows:

Case 1: If $u_{1} \in V_{1}\left(\bar{M}_{0}\right) \cup V_{2}\left(\bar{M}_{0}\right) \cup\left(V_{3}\left(\bar{M}_{0}\right) \backslash V_{3}^{3}\right)$. In this region, we define the following vector field:

$$
W_{4}^{1}:=W_{4}^{0}+\left(1 / M_{2}^{2}\right) \widetilde{W}\left(u_{1}\right)
$$

where $\widetilde{W}$ is the convex combination of the pseudogradients constructed in $V_{1}\left(\bar{M}_{0}\right), V_{2}\left(\bar{M}_{0}\right)$ and $V_{3}\left(\bar{M}_{0}\right) \backslash V_{3}^{3}$. It follows then that

$$
\begin{aligned}
\left\langle-\nabla J_{K}(u), W_{4}^{1}\right\rangle \geq & \bar{\Gamma}_{4}+\frac{1}{M_{2}^{2}}\left(\sum_{i \in I} \frac{c}{\mu_{i}}+c \sum_{i \in I \cap \mathcal{I}_{b}}\left|1-J_{K}(u)^{\frac{n}{n-2}} \alpha_{i}^{\frac{4}{n-2}} K\left(a_{i}\right)\right|\right. \\
& \left.+c \sum_{k \neq r ; k, r \in I} \varepsilon_{k r}+c \sum_{i \in I \cap \mathcal{I}_{i n}} \frac{\left|\nabla K\left(a_{i}\right)\right|}{\lambda_{i}}+O\left(\sum_{j \in I ; \ell \notin I} \varepsilon_{j \ell}\right)\right) .
\end{aligned}
$$

To complete the proof, it remains to absorb the last term. To this aim, we notice that:

(i) if " $\ell \in \mathcal{I}_{\text {in }}$ with $\ell \geq i_{0}$ or $\ell \in \mathcal{I}_{b}$ with $\ell \geq j_{0}$ ", then the term $\varepsilon_{j \ell}$ is already in $\bar{\Gamma}_{4}$ the lower bound of (41). Taking $M_{2}$ large, we will be able to absorb this term.

(ii) if " $\ell \in \mathcal{I}_{i n}$ with $\ell<i_{0}$ or $\ell \in \mathcal{I}_{b}$ with $\ell<j_{0}$ ", then there holds: $\varepsilon_{j \ell} \leq c \frac{M_{2}}{\mu_{\ell}} \leq$ $c\left(M_{2} / M_{0}^{1 /(q+p-1)}\right) \frac{1}{\mu_{k_{0}}}=o\left(1 / \mu_{k_{0}}\right)$ by choosing $M_{2} / M_{0}^{1 /(q+p-1)}$ small enough (see (31)) and where $k_{0}:=\max I$. Hence, we are also able to remove this term. (Recall that, in Lemmas 3.5-3.8, 3.13, the constant over $\mu_{\max }$ is independent of $M_{0}$ and $M_{2}$ ). Hence the estimate in the first case follows as in the proof of the previous lemmas.

Case 2: In this case we take $u_{1} \in V_{3}^{3}\left(\bar{M}_{0}\right)$ and assume that $D_{9} \cup D_{8} \neq \emptyset$, where

$$
D_{9}:=\left\{i \in I: i \in B_{z} \text { with } \# B_{z}=1\right\} ; \quad D_{8}:=I \cap \mathcal{I}_{\text {in }} .
$$

Here we define the following vector field:

$$
W_{4}^{2}:=W_{4}^{0}+\left(1 / M_{2}^{2}\right) \sum_{i \in D_{8} \cup D_{9}} \lambda_{i} \frac{\partial \varphi_{i}}{\partial \lambda_{i}} .
$$

We point out that, this pseudogradient increases the $\mu_{i}$ for $i \in D_{8} \cup D_{9}$, but does not increase the $\mu_{\max }:=\mu_{p+q}$ since $p+q \notin I$. Furthermore observe that

$$
\left\langle-\nabla J_{K}(u), \sum_{i \in D_{8} \cup D_{9}} \lambda_{i} \frac{\partial \varphi_{i}}{\partial \lambda_{i}}\right\rangle \geq c \sum_{i \in D_{8} \cup D_{9}}\left(\frac{1}{\mu_{i}}+O\left(\sum_{j=1}^{p+q} \frac{1}{\lambda_{j}^{3}}+\sum_{\ell \notin I} \varepsilon_{i \ell}\right)\right) .
$$

Hence the result follows as the first case.

Next we set

$$
D_{10}:=\left\{i \in I: \sum_{k \in I ; k \neq i} \varepsilon_{k i} \leq m_{1} q / \lambda_{i}\right\} \neq \emptyset \text {, where } m_{1} \text { is a small constant. }
$$


Case 3: In this case we take $u_{1} \in V_{3}^{3}\left(\bar{M}_{0}\right)$ and assume that $D_{9} \cup D_{8}=\emptyset$. That is we have that $I \subset \mathcal{I}_{b}$ and that $\# B_{z} \neq 1$ for each $z$ critical point of $K_{1}$. Furthermore we assume that $D_{10} \neq \varnothing$.

Next we recall that in this case, for each $z$ such that $\# B_{z} \geq 2, z$ has to be a local maximum point with $\partial K / \partial v>0$ (which implies that $\mu_{i}$ and $\lambda_{i}$ are of the same order). Hence one can use the same pseudogradient defined in Case 2 (by replacing $D_{8} \cup D_{9}$ by $D_{10}$ ). Hence for $i \in D_{10}$, using Proposition 5.4, we derive that

$$
\left\langle-\nabla J_{K}(u), \lambda_{i} \frac{\partial \delta_{i}}{\partial \lambda_{i}}\right\rangle \geq \frac{c}{\lambda_{i}}+O\left(\sum_{j \neq i} \varepsilon_{i j}\right) \geq \frac{c}{\lambda_{i}}+c \sum_{j \neq i ; j \in I} \varepsilon_{i j}+O\left(\sum_{j \notin I} \varepsilon_{i j}\right)
$$

and the proof follows as the previous cases.

Case 4: $u_{1} \in V_{3}^{3}\left(\bar{M}_{0}\right)$ and $I \subset I_{b}, \# B_{z} \neq 1$ for each $z$ and $D_{10}=\emptyset$.

In this case, for each $z$ such that $\# B_{z} \geq 2, z$ has to be a local maximum point with $\partial K / \partial v>0$ (which implies that $\mu_{i}$ and $\lambda_{i}$ are of the same order). Let $z_{1}, \ldots, z_{\ell}$ be such that $\# B_{z_{j}} \geq 2$. Thus, the function $u$ can be written as

$$
u:=\sum_{j=1}^{\ell} u_{j}+u_{\ell+1} \quad \text { where } \quad u_{j}:=\sum_{i \in B_{z_{j}}} \alpha_{i} \varphi_{i} \text { for } j \leq \ell \quad \text { and } \quad u_{\ell+1}:=\sum_{i \notin I} \alpha_{i} \varphi_{i} .
$$

Notice that, for $j \leq \ell$, it follows that $u_{j} \in V\left(z_{j}, \# B_{z_{j}}, \eta, \varepsilon, \bar{M}_{0}\right)$ and in Lemma 3.9, we have constructed a pseudogradient $W\left(z_{j}, \# B_{z_{j}}\right)$ in this region. Now, we define

$$
W_{4}^{4}:=W_{4}^{0}+\frac{1}{M_{2}^{2}} \sum_{j=1}^{\ell} W\left(z_{j}, \# B_{z_{j}}\right)\left(u_{j}\right) .
$$

Observe that, by Lemma 3.9, we have

$$
\begin{aligned}
& \left\langle-\nabla J_{K}(u), W\left(z_{j}, \# B_{z_{j}}\right)\left(u_{j}\right)\right\rangle \\
& \quad \geq c \sum_{k \in B_{z_{j}}}\left(\sum_{r \neq k ; r \in B_{z_{j}}} \varepsilon_{k r}^{\frac{n-1}{n-2}}+O\left(\sum_{r \notin B_{z_{j}}, r \in \mathcal{I}_{i n}} \varepsilon_{k r}+\sum_{r \notin B_{z_{j}}, r \in \mathcal{I}_{b}} \frac{1}{\lambda_{k}}\left|\frac{\partial \varepsilon_{k r}}{\partial a_{k}}\right|\right)\right) .
\end{aligned}
$$

Furthermore we notice that, for $r \notin B_{z_{j}}$ and $r \in \mathcal{I}_{i n},(i)$ either $r \geq i_{0}$ and therefore the $\varepsilon_{k r}$ exists already in $\bar{\Gamma}_{4}$ or $(i i) r<i_{0}$ and, using Lemma 3.3, it follows that $a_{r}$ is close to a critical point $y$ of $K$ in $\mathbb{S}_{+}^{n}$ which implies that $\varepsilon_{k r} \leq c\left(1 / \lambda_{k}^{n-2}+1 / \lambda_{r}^{n-2}\right)$. Next for $r \notin B_{z_{j}}$ and $r \in \mathcal{I}_{b}$, three situations may occur

(i) $r \geq j_{0}$ and therefore the $\varepsilon_{k r}$ exists already in $\bar{\Gamma}_{4}$.

(ii) $r<j_{0}$ and $r \notin I$. In this case it follows that $\varepsilon_{k r} \leq M_{2} / \lambda_{r}$ and thus (since $\lambda_{r} \geq$ $M_{0}^{1 / q+p-1} \lambda_{k}$ for each $k \in I$ ) we have that

$$
\begin{aligned}
\frac{1}{\lambda_{k}}\left|\frac{\partial \varepsilon_{k r}}{\partial a_{k}}\right| & \leq c \lambda_{r} d\left(a_{r}, a_{k}\right) \varepsilon_{k r}^{\frac{n}{n-2}} \leq c \sqrt{\frac{\lambda_{r}}{\lambda_{k}}} \varepsilon_{k r}^{\frac{n-1}{n-2}} \leq \frac{c M_{2}^{\frac{n-1}{n-2}}}{\lambda_{k}^{1 / 2} \lambda_{r}^{1 / 2+1 /(n-2)}} \\
& \leq c \frac{M_{2}^{\frac{n-1}{n-2}}}{\left.M_{0}^{(1 / 2+1 /(n-2)}\right) / q+p-1} \frac{1}{\lambda_{k}^{1+1 /(n-2)}}=o\left(\left(\frac{m_{1}}{\lambda_{k}}\right)^{(n-1) /(n-2)}\right)
\end{aligned}
$$

(by using (31)). 
(iii) $r<j_{0}$ and $r \in I$. In this case, it follows that $a_{r} \in B_{z_{\ell}}$ with $\ell \neq k$ and therefore we deduce that $\left|a_{k}-a_{r}\right| \geq c>0$. Hence we get

$$
\frac{1}{\lambda_{k}}\left|\frac{\partial \varepsilon_{k r}}{\partial a_{k}}\right|=O\left(\frac{1}{\lambda_{k}^{n-1}}+\frac{1}{\lambda_{r}^{n-1}}\right) .
$$

Using (41),(43), the previous estimates and the fact that $D_{10}=\emptyset$, the lemma follows in this case.

Proof of Proposition 3.2 The required pseudogradient will be a convex combination of the ones defined in the previous lemmas. Each one is bounded and satisfies Claim $(i)$. Furthermore, the only case where $\mu_{\max }$ increases is the region $\mathcal{W}$. Finally, Claim ( $i i$ ) follows from the first one and the estimate of $\|\bar{v}\|^{2}$ which is small with respect to the lower bound of Claim (i). Concerning the last claim, it follows easily from the definition of the pseudogradient. This achieves the proof of Proposition 3.2.

\subsubsection{Critical points at infinity and their topological contribution}

For $\varepsilon_{0}$ a small number, we define the following neighborhood of the cone of positive solutions of the sphere in $H^{1}\left(\mathbb{S}_{+}^{n}\right)$ :

$V_{\varepsilon_{0}}\left(\Sigma^{+}\right):=\left\{u \in \Sigma ; J_{K}(u)^{(2 n-2) /(n-2)} e^{2 J(u)}\left|u^{-}\right|_{L^{2 n /(n-2)}}^{4 /(n-2)}<\varepsilon_{0}\right\}, \quad$ where $u^{-}:=\max (0,-u)$.

This set is for $\varepsilon_{0}$ small enough invariant under the gradient flow lines of the Euler Lagrange functional $J_{K}$. Namely we prove that

Lemma 3.15 For $\varepsilon_{0}>0$ small enough, the set $V_{\varepsilon_{0}}\left(\Sigma^{+}\right)$is invariant under the flow generated by $-\nabla J_{K}$.

Proof We will write $J$ instead of $J_{K}$. For $w \in L^{2 n /(n+2)}\left(\mathbb{S}_{+}^{n}\right)$, we denote by $\mathcal{L}^{-1}(w)$ the solution of the following PDE:

$$
\left\{\begin{array}{l}
\mathcal{L} u:=-\Delta u+\frac{n(n-2)}{4} u=w \text { in } \mathbb{S}_{+}^{n}, \\
\partial u / \partial v=0 \text { on } \partial \mathbb{S}_{+}^{n} .
\end{array}\right.
$$

Furthermore, it holds

$$
\begin{aligned}
& |u|_{L^{2 n /(n-2)}} \leq c\|u\|_{H^{1}} \leq c|w|_{L^{2 n /(n+2)}} \\
& \left|\mathcal{L}^{-1}\left(K|u|^{4 /(n-2)} u\right)\right|_{L^{2 n /(n-2)}} \leq c|u|_{L^{2 n /(n-2)}}^{(n+2) /(n-2)}
\end{aligned}
$$

Suppose $u_{0} \in V_{\varepsilon_{0}}\left(\Sigma^{+}\right)$and consider

$$
\left\{\begin{array}{l}
\frac{d u(s)}{d s}=-\nabla J(u(s))=-2 J(u)\left(u-J(u)^{n /(n-2)} \mathcal{L}^{-1}\left(K|u|^{4 /(n-2)} u\right)\right) \\
u(0)=u_{0} .
\end{array}\right.
$$

Then

$$
\begin{aligned}
& e^{2 \int_{0}^{s} J(u(t)) d t} u(s)=u_{0}+2 \int_{0}^{s} e^{2 \int_{0}^{t} J(u(y)) d y} J(u(t))^{\frac{2 n-2}{n-2}} \mathcal{L}^{-1}\left(K|u(t)|^{4 /(n-2)} u(t)\right) d t, \\
& u^{-}(s) \leq e^{-2 \int_{0}^{s} J(u(t)) d t}\left(u_{0}^{-}+2 \int_{0}^{s} e^{2 \int_{0}^{t} J(u(y)) d y} J(u(t))^{\frac{2 n-2}{n-2}} \mathcal{L}^{-1}\left(K\left(u^{-}(t)\right)^{\frac{n+2}{n-2}}\right) d t\right) \\
& :=e^{-2 \int_{0}^{s} J(u)} f(s) .
\end{aligned}
$$


Setting

$$
F(s)=e^{-\frac{4 n}{n-2} \int_{0}^{s} J(u(t)) d t}|f(s)|_{L^{2 n /(n-2)}}^{2 n /(n-2)} \quad \text { which implies that } \quad\left|u^{-}(s)\right|_{L^{2 n /(n-2)}}^{2 n /(n-2)} \leq F(s) .
$$

Recall that, if $u_{0}^{-}=0$ then $u(s)$ is positive for all $s$. Hence, we can assume that $u_{0}^{-} \neq 0$ and we want to prove that $F$ is a decreasing function. Observe that

$$
\begin{aligned}
F^{\prime}(s) & =-\frac{4 n}{n-2} J(u(s)) e^{-\frac{4 n}{n-2} \int_{0}^{s} J(u)}|f(s)|_{L^{2 n /(n-2)}}^{2 n /(n-2)}+e^{-\frac{4 n}{n-2} \int_{0}^{s} J(u)} \frac{2 n}{n-2} \int_{\mathbb{S}_{+}^{n}} f^{\prime}(s) f(s)^{\frac{n+2}{n-2}} d x \\
& \left.\leq \frac{2 n}{n-2} e^{-\frac{4 n}{n-2} \int_{0}^{s} J(u)}\left[-2 J(u(s))\left|u_{0}^{-}\right|_{L^{2 n /(n-2)}}^{2 n /(n-2)}+\int_{\mathbb{S}_{+}^{n}} f^{\prime}(s) f(s)^{\frac{n+2}{n-2}} d x\right] \quad \text { (using } f(s) \geq u_{0}^{-}\right) .
\end{aligned}
$$

Notice that $f^{\prime}(0)=u_{0}^{-}$and therefore

$$
\left|\int_{\mathbb{S}_{+}^{n}} f^{\prime}(s) f(s)^{\frac{n+2}{n-2}}\right| d x \leq c \int_{\mathbb{S}_{+}^{n}}\left|f^{\prime}(s)\right|\left|u_{0}^{-}\right|^{\frac{n+2}{n-2}}+c \int_{\mathbb{S}_{+}^{n}}\left|f^{\prime}(s)\right|\left(\int_{0}^{s}\left|f^{\prime}(t)\right| d t\right)^{\frac{n+2}{n-2}} d x .
$$

But, we have (using (45))

$$
\begin{aligned}
\int_{\mathbb{S}_{+}^{n}}\left(u_{0}^{-}\right)^{\frac{n+2}{n-2}}\left|f^{\prime}(s)\right| d x & =\int_{\mathbb{S}_{+}^{n}}\left(u_{0}^{-}\right)^{\frac{n+2}{n-2}}\left(2 e^{2 \int_{0}^{s} J(u)} J(u(s))^{\frac{2 n-2}{n-2}} \mathcal{L}^{-1}\left(K\left(u^{-}(s)\right)^{\frac{n+2}{n-2}}\right)\right) d x \\
& \leq C J(u(s))^{\frac{2 n-2}{n-2}} e^{2 \int_{0}^{s} J(u)}\left|u_{0}^{-}\right|_{L^{2 n /(n-2)}}^{(n+2) /(n-2)}\left|u^{-}(s)\right|_{L^{2 n /(n-2)}}^{(n+2) /(n-2)},
\end{aligned}
$$

and we also have (using the fact that $J(u(s))$ is a decreasing function)

$$
\begin{aligned}
\int_{\mathbb{S}_{+}^{n}}\left|f^{\prime}(s)\right| & \left.\left(\int_{0}^{s} \mid f^{\prime}(t)\right) \mid d t\right)^{\frac{n+2}{n-2}} d x \leq c s^{\frac{4}{n-2}} \int_{\mathbb{S}_{+}^{n}}\left|f^{\prime}(s)\right| \int_{0}^{s}\left|f^{\prime}(t)\right|^{\frac{n+2}{n-2}} d t d x \\
& \leq c s^{\frac{4}{n-2}} e^{\frac{4 n}{n-2} s J\left(u_{0}\right)} J\left(u_{0}\right)^{\frac{2 n-2}{n-2} \frac{2 n}{n-2}}\left|u^{-}(s)\right|_{L^{2 n /(n-2)}}^{(n+2) /(n-2)} \int_{0}^{s}\left|u^{-}(t)\right|_{L^{2 n /(n-2)}}^{(n+2)^{2} /(n-2)^{2}} d t .
\end{aligned}
$$

Hence, if $\left|u^{-}(s)\right|_{L^{2 n /(n-2)}} \leq 5\left|u_{0}^{-}\right|_{L^{2 n /(n-2)}}$, for $0 \leq s \leq 1$, we derive that

$$
\begin{aligned}
F^{\prime}(s) \leq \frac{4 n}{n-2} e^{-\frac{4 n}{n-2} \int_{0}^{s} J(u)}\left|u_{0}^{-}\right|_{L^{2 n /(n-2)}}^{2 n /(n-2)}( & -J(u(s))+c J\left(u_{0}\right)^{\frac{2 n-2}{n-2}} e^{2 J\left(u_{0}\right)}\left|u_{0}^{-}\right|_{L^{2 n /(n-2)}}^{4 /(n-2)} \\
& \left.+c\left(J\left(u_{0}\right)^{\frac{2 n-2}{n-2}} e^{2 J\left(u_{0}\right)}\left|u_{0}^{-}\right|_{L^{2 n /(n-2)}}^{4 /(n-2)}\right)^{2 n /(n-2)}\right)
\end{aligned}
$$

Finally, since inf $J>c>0$, using the fact that $u_{0} \in V_{\varepsilon_{0}}\left(\Sigma^{+}\right)$, that is, $J\left(u_{0}\right)^{\frac{2 n-2}{n-2}} e^{2 J\left(u_{0}\right)}$ $\left|u_{o}^{-}\right|_{L^{2 n /(n-2)}}^{4 /(n-2)}<\varepsilon_{0}$, and $\eta$ is small enough, then $F^{\prime}(s) \leq 0$, for $0 \leq s \leq 1$. Therefore $J(u(s))^{\frac{2 n-2}{n-2}} e^{2 J(u(s))}\left|u(s)^{-}\right|_{L^{2 n /(n-2)}}^{4 /(n-2)}<\varepsilon_{0}$, and our result follows.

Next using a partition of the unity, one can define the vector field $W$ of Proposition 3.2 globally by gluing it to the negative gradient $-\nabla J$ outside the $V(q, p, m, \varepsilon)$ 's. Let us denote the resulting global vector field by $Y$ and define a new vector field by setting:

$$
X(u):=Y(u)-<Y(u), u>u \quad \text { for } u \in V_{\varepsilon_{0}}\left(\Sigma^{+}\right) .
$$

We then have

Corollary 3.16 Assume that $K$ satisfies (H1), (H2) and (H3). Then using Propositions 3.1, 3.2 and arguing as in the above Lemma, one proves that for $\varepsilon_{0}$ small enough, $X$ is a pseudogradient of $J$ which preserves $V_{\varepsilon_{0}}\left(\Sigma^{+}\right)$. Moreover the critical points at infinity of $X$ lie in subsets $\mathcal{W}$ (see the formula (26) for a definition) 
Next we perform a Morse type reduction in the subsets $\mathcal{W}$. Namely we prove

Lemma 3.17 For $u=\sum_{i=1}^{q} \alpha_{i} \delta_{a_{i}, \lambda_{i}}+\sum_{q+1}^{p+q} \alpha_{i} \varphi_{a_{i}, \lambda_{i}} \in \mathcal{W}$, we define $D_{4}:=\left\{i \leq q: a_{i}\right.$ is close to $z$ with $\left.\frac{\partial K}{\partial v}(z)=0\right\} \& D_{5}:=\left\{i \leq q: a_{i}\right.$ is close to $z$ with $\left.\frac{\partial K}{\partial v}(z)>0\right\}$.

Then the functional $J_{K}$ expands as follows

$$
\begin{aligned}
J_{K}(u)= & \frac{\left(\sum_{i \leq q} \alpha_{i}^{2}+2 \sum_{i>q} \alpha_{i}^{2}\right) S_{n}^{2 / n}}{\left(\sum_{i \leq q} \alpha_{i}^{\frac{2 n}{n-2}} K\left(a_{i}\right)+2 \sum_{i>q} \alpha_{i}^{\frac{2 n}{n-2}} K\left(a_{i}\right)\right)^{\frac{n-2}{n}}}\left(1-c \sum_{i>q} \frac{\Delta K\left(y_{i}\right)}{\lambda_{i}^{2}}+c \sum_{i \in D_{5}} \frac{1}{\lambda_{i}} \frac{\partial K}{\partial v}\left(z_{i}\right)\right. \\
& \left.+c \sum_{i \in D_{4}}\left(\frac{c_{7}}{\lambda_{i}} \frac{\partial K}{\partial v}\left(a_{i}\right)-c_{6} \frac{\Delta K\left(a_{i}\right)}{\lambda_{i}^{2}}\right)+o\left(\sum_{i \in D_{5}} \frac{1}{\lambda_{i}}+\sum_{i \in D 4} \frac{1}{\lambda_{i}^{2}}+\sum_{i>q} \frac{1}{\lambda_{i}^{2}}\right)\right) \\
= & S_{n}^{2 / n}\left(\sum_{i \leq q} \frac{1}{K\left(z_{i}\right)^{\frac{n-2}{2}}}+2 \sum_{i>q} \frac{1}{K\left(y_{i}\right)^{\frac{n-2}{2}}}\right)^{\frac{2}{n}}\left(1-\|\alpha\|^{2}+\sum_{i=1}^{p+q}\left(\left|A_{i}^{-}\right|^{2}-\left|A_{i}^{+}\right|\right)-c \sum_{i>q} \frac{\Delta K\left(y_{i}\right)}{\lambda_{i}^{2}}\right. \\
& \left.+c \sum_{i \in D_{5}} \frac{1}{\lambda_{i}} \frac{\partial K}{\partial v}\left(z_{i}\right)+c \sum_{i \in D_{4}}\left(\frac{c_{7}}{\lambda_{i}} \frac{\partial K}{\partial v}\left(a_{i}\right)-c_{6} \frac{\Delta K\left(z_{i}\right)}{\lambda_{i}^{2}}\right)+o\left(\sum_{i \in D_{5}} \frac{1}{\lambda_{i}}+\sum_{[i>q] \cup\left[i \in D_{4}\right]} \frac{1}{\lambda_{i}^{2}}\right)\right),
\end{aligned}
$$

where $S_{n}$ is defined in Proposition 5.3 (it represents the level of one boundary bubble), $\alpha \in \mathbb{R}^{q+p-1},\left(A_{i}^{+}, A_{i}^{-}\right)$are the local coordinates of the parameters $\left(\alpha_{1}, \ldots, \alpha_{p+q}\right)$ and $a_{i}$. This expansion will be called the Morse Lemma at Infinity of $J_{K}$ near its critical point at infinity. Note that we loose an index for the parameter $\alpha$ since the functional $J_{K}$ is homogenous with respect to this parameter.

From Propositions 3.1, 3.2 and Lemma 3.17, we derive the characterization of critical points at infinity and identify their level sets. Namely we have:

Corollary 3.18 Assume that $K$ satisfies (H1), (H2) and (H3). Then, in $V(m, q, p, \varepsilon)$, the critical points at infinity of $J_{K}$ are in one to one correspondence with the collections of $q$ critical points $z_{\ell}$ 's of $K_{1}$ satisfying: either $z_{\ell}$ is a local maximum point with $\partial K / \partial v\left(z_{\ell}\right)>0$ or $\partial K / \partial v\left(z_{\ell}\right)=0$ and $\Delta K\left(z_{\ell}\right)<0$ and $p$ critical points $y_{r}$ 's of $K$ in $\mathbb{S}_{+}^{n}$ satisfying $\Delta K\left(y_{r}\right)<$ 0 . We will denote such a critical point at infinity by $\left(z_{1}, \ldots, z_{q}, y_{q+1}, \ldots, y_{q+p}\right)_{\infty}$. Such a critical point at infinity is at the level (see (46))

$$
C_{\infty}\left(z_{1}, \ldots, z_{q}, y_{q+1}, \ldots, y_{q+p}\right):=S_{n}^{2 / n}\left(\sum_{i=1}^{q} \frac{1}{K\left(z_{i}\right)^{(n-2) / 2}}+\sum_{i=q+1}^{q+p} \frac{2}{K\left(y_{i}\right)^{(n-2) / 2}}\right)^{2 / n} .
$$

In particular, it holds that

$$
C_{\min }^{(2 p+q), \infty}:=\frac{\left((2 p+q) S_{n}\right)^{2 / n}}{K_{\max }^{(n-2) / n}} \leq C_{\infty}\left(z_{1}, \ldots, z_{q}, y_{q+1}, \ldots, y_{q+p}\right) \leq \frac{\left((2 p+q) S_{n}\right)^{2 / n}}{K_{\min }^{(n-2) / n}}:=C_{\max }^{(2 p+q), \infty}
$$

Furthermore, for such a critical point at infinity, we associate an index (which corresponds to the number of the decreasing directions for $J_{K}$ by using the Morse Lemma at infinity, see (46))

$$
\begin{aligned}
i_{\infty}\left(z_{1}, \ldots, z_{q}, y_{q+1}, \ldots, y_{q+p}\right) & :=q+p-1+\sum_{i=1}^{q}\left(n-1-\operatorname{morse}\left(K_{1}, z_{i}\right)\right) \\
& +\sum_{i=q+1}^{q+p}\left(n-\operatorname{morse}\left(K, y_{i}\right)\right) .
\end{aligned}
$$


Such an index will be called the $i_{\infty}$-index of such a critical point at infinity.

Next as consequence of the above corollary and the Morse reduction in Lemma 3.17 we compute the topological contribution of the critical points at infinity to the difference of topology between the level sets of the functional $J_{K}$. Namely we have

Lemma 3.19 Let $\tau_{\infty}$ be a critical point at infinity at the level $C_{\infty}\left(\tau_{\infty}\right)$ with index $i_{\infty}\left(\tau_{\infty}\right)$. Then for $\theta$ a small positive number and a field $\mathbb{F}$, we have that

$$
H_{l}\left(J_{K}^{C_{\infty}\left(\tau_{\infty}\right)+\theta}, J_{K}^{C_{\infty}\left(\tau_{\infty}\right)-\theta} ; \mathbb{F}\right)=\left\{\begin{array}{l}
\mathbb{F} \quad \text { if } l=i_{\infty}\left(\tau_{\infty}\right), \\
0, \text { otherwise. }
\end{array}\right.
$$

where $H_{l}$ denotes the $l$-dimensional homology group with coefficient in the field $\mathbb{F}$.

\section{Proof of the main results}

This section is devoted to the proof of Theorems 1.1,1.3 and 1.4. The proof of these theorems is based on the characterization of the critical points at infinity in Corollary 3.18 and the computation of their contribution to the difference of topology in Lemma 3.19. It also uses two deformation lemmas. The first one is an abstract lemma, which is inspired by Proposition 3.1 in [35]. It reads as follows:

Lemma 4.1 Let $\underline{A}$ and $\bar{A}:=\left(K_{\max } / K_{\min }\right)^{(n-2) / n} \underline{A}$. Assume that $J_{K}$ does not have any

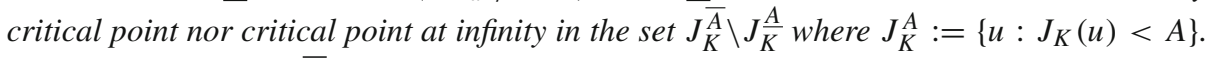
Then for each $c \in[\underline{A}, \bar{A}]$, the level set $J_{K}^{c}$ is contractible.

Proof First, since we assumed that $J_{K}$ does not have any critical point nor critical point at infinity in $\Sigma^{+}$between the levels $\bar{A}$ and $\underline{A}$, we have that $J_{K}^{\bar{A}}$ retracts by deformation onto

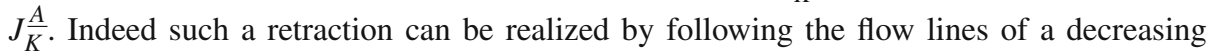
pseudogradient $Z_{K}$ for $J_{K}$. Let $\phi_{K}$ denote the one parameter group corresponding to this pseudogradient. For each $u \in \Sigma^{+}$, we denote by $s_{K}(u)$ the first time such that $\phi_{K}\left(s_{K}(u), u\right) \in$ $J \frac{A}{K}$.

Secondly we recall that, for $K \equiv 1$, the only critical points of $J_{1}$ are minima and lie in the bottom level $S_{n}$. Furthermore, for each $A>S_{n}$, the set $J_{1}^{A}$ is a contractible one. Indeed by following the flow lines of a decreasing pseudogradient $Z_{1}$ of the Yamabe functional $J_{1}$, each flow line, starting from $u \in \Sigma^{+}$, will reach the bottom level $S_{n}$. Let us denote by $\phi_{1}$ the one parameter group corresponding to $Z_{1}$.

Next we notice that, we have

$$
\left(1 / K_{\max }^{(n-2) / n}\right) J_{1}(u) \leq J_{K}(u) \leq\left(1 / K_{\min }^{(n-2) / n}\right) J_{1}(u) \quad \text { for each } u \in \Sigma,
$$

which implies that

$$
J \frac{A}{K} \subset J_{1}^{A^{\prime}} \subset J_{K}^{\bar{A}} \quad \text { where } A^{\prime}:=K_{\max }^{(n-2) / n} \underline{A} .
$$

Furthermore we observe that for each $u \in \Sigma^{+}$, there exists a unique $s_{1}(u)$ satisfying $\phi_{1}\left(s_{1}(u), u\right) \in J_{1}^{A^{\prime}}$.

Next we define the following map:

$$
F:=[0,1] \times J_{1}^{A^{\prime}} \rightarrow J_{1}^{A^{\prime}} ; \quad F(t, u):=\phi_{1}\left(s_{1}\left(\phi_{K}\left(t s_{K}(u), u\right)\right), \phi_{K}\left(t s_{K}(u), u\right)\right) .
$$

We notice that $F$ is well defined and continuous and satisfies the following properties: 
- For $t=0$, we have $\phi_{K}(0, u)=u$. Furthermore, for each $u \in J_{1}^{A^{\prime}}$, we have $s_{1}(u)=0$. Therefore, for each $u \in J_{1}^{A^{\prime}}$, we get $F(0, u)=\phi_{1}(0, u)=u$.

- For $t=1$, we have $\phi_{K}\left(s_{K}(u), u\right) \in J_{K}^{A} \subset J_{1}^{A^{\prime}}$ (by the definition of $s_{K}$ ) which implies that $s_{1}\left(\phi_{K}\left(s_{K}(u), u\right)\right)=0$ and therefore $F(1, u)=\phi_{1}\left(0, \phi_{K}\left(s_{K}(u), u\right)\right)=\phi_{K}\left(s_{K}(u), u\right) \in$ $J \frac{A}{K}$ for each $u \in J_{1}^{A^{\prime}}$.

- If $u \in J \frac{A}{K}$, then $s_{K}(u)=0$ which implies that $\phi_{K}\left(t s_{K}(u), u\right)=\phi_{K}(0, u)=u$. Therefore $F(t, u)=\phi_{1}\left(s_{1}(u), u\right)=\phi_{1}(0, u)=u$ for each $u \in J_{K}^{A}$ and each $t \in[0,1]$ (we used $s_{1}(u)=0$ since $\left.u \in J \frac{A}{K} \subset J_{1}^{A^{\prime}}\right)$.

Thus $J_{1}^{A^{\prime}}$ retracts by deformation onto $J_{K}^{A}$, a fact which provides the claim of the lemma since $J_{1}^{A^{\prime}}$ itself is a contractible set.

The second deformation lemma is a consequence of the previous one, the assumptions $(H 1),(H 2),(H 3)$ of this paper and an appropriate pinching condition for the function $K$. To state it we set the following notation:

$$
\text { for } \ell \in \mathbb{N}, \quad C_{\max }^{\ell, \infty}:=\left(\ell S_{n}\right)^{2 / n} / K_{\min }^{(n-2) / n} \& \quad C_{\min }^{\ell, \infty}:=\left(\ell S_{n}\right)^{2 / n} / K_{\max }^{(n-2) / n} .
$$

We recall that it follows from Corollary 3.18 that the level of critical points at Infinity corresponding to $q$ boundary points and $p$ interior points such that $q+2 p=\ell$ lie between $C_{\min }^{\ell, \infty}$ and $C_{\max }^{\ell, \infty}$.

Our second deformation lemma reads as follows:

Proposition 4.2 For $k \in \mathbb{N}$ a fixed integer, let $0<K \in C^{3}\left(\overline{\mathbb{S}_{+}^{n}}\right)$ satisfying the conditions (H1), (H2), (H3) and the pinching condition $K_{\max } / K_{\min }<((k+1) / k)^{1 /(n-2)}$.

Assume that $J_{K}$ does not have any critical point under the level $C_{\mathrm{min}}^{k+1, \infty}$. Then, for every $1 \leq \ell \leq k$ and every $c \in\left(C_{\max }^{\ell, \infty}, C_{\min }^{\ell+1, \infty}\right)$, the sublevel $J_{K}^{c}$ is a contractible set.

Proof Since we assumed that $K_{\max } / K_{\min }<((k+1) / k)^{1 /(n-2)}$, it follows that, for each $1 \leq \ell \leq k$, we have $(k+1) / k \leq(\ell+1) / \ell$ and

$$
C_{\max }^{\ell, \infty}<C_{\max }^{\ell, \infty}\left(K_{\max } / K_{\min }\right)^{(n-2) / n}<C_{\min }^{\ell+1, \infty} .
$$

The proof follows then from Lemma 4.1 by taking $\underline{A}=C_{\max }^{\ell, \infty}+\gamma$ with a small $\gamma>0$ so that $\bar{A}<C_{\text {min }}^{\ell+1, \infty}$. Indeed between the levels $\underline{A}$ and $\bar{A}$ the functional $J_{K}$ does not have any critical point nor critical point at infinity.

Next we start the proof of our existence results by proving Theorem 1.3.

Proof of Theorem 1.3 Arguing by contradiction we assume that the functional $J_{K}$ does not have any critical point under the level $C_{\mathrm{min}}^{2, \infty}$. Hence it follows from Proposition 4.2 (with $k=1$ ) that under the assumption of Theorem 1.3, we have that $J^{C_{\max }^{1, \infty}+\gamma}$ is a contractible set, for $\gamma$ a small constant. Moreover it is a retract by deformation of $C_{\min }^{2, \infty}$. Furthermore follows from corollary 3.18 that critical points at infinity under the level $C_{\mathrm{min}}^{2, \infty}$ are in one to one correspondence with critical points of $K_{1}$ in $\mathcal{K}_{b}^{+} \cup \mathcal{K}_{b}^{0,-}$. Then it follows from Lemma 3.19 and the Euler-Poincaré theorem that:

$$
1=\chi\left(J^{C_{\min }^{2, \infty}+\gamma}\right)=\sum_{z \in \mathcal{K}_{b}^{+} \cup \mathcal{K}_{b}^{0,-}}(-1)^{n-1-\operatorname{morse}\left(K_{1}, z\right)}
$$

which contradicts the assumption $(b)$ of Theorem 1.3. Hence the existence of at least one critical point of $J_{K}$. 
Proof of Theorem 1.4 Assuming that $J_{K}$ does not have any critical point under the level $C_{\mathrm{min}}^{3, \infty}$, we derive, using Proposition 4.2 (with $k=2$ ), the level sets $J_{K}^{C_{\max }^{1, \infty}+\gamma}$ and $J_{K}^{C_{\max }^{2, \infty}+\gamma}$ are contractible sets. Then it follows from the properties of the Euler-Characteristic, see Proposition 5.7 , pp.105 in [26], that

$$
1=\chi\left(J_{K}^{C_{\max }^{2, \infty}+\gamma}\right)=\chi\left(J_{K}^{C_{\max }^{2, \infty}+\gamma}, J_{K}^{C_{\max }^{1, \infty}+\gamma}\right)+\chi\left(J_{K}^{C_{\max }^{1, \infty}+\gamma}\right) .
$$

That is $\chi\left(J_{K}^{C_{\max }^{2, \infty}+\gamma}, J_{K}^{C_{\max }^{1, \infty}+\gamma}\right)=0$. Moreover it follows from Corollary 3.18 that the critical points at infinity between these two levels are $\left(z_{i}, z_{j}\right)_{\infty}$ with $z_{i} \neq z_{j} \in \mathcal{K}_{b}^{+} \cup \mathcal{K}_{b}^{0,-}$ and $y_{\infty}$ with $y \in \mathcal{K}_{i n}^{-}$. Thus, it follows from Lemma 3.19 and the Euler-Poincaré theorem that

$$
\sum_{z_{i} \neq z_{j} \in \mathcal{K}_{b}^{+} \cup \mathcal{K}_{b}^{0,-}}(-1)^{1+\iota\left(z_{i}\right)+\iota\left(z_{j}\right)}+\sum_{y \in \mathcal{K}_{i n}^{-}}(-1)^{\iota(y)}=0
$$

where $\iota\left(z_{k}\right):=n-1-\operatorname{morse}\left(K_{1}, z_{k}\right)$ and $\iota(y):=n-\operatorname{morse}(K, y)$.

Observe that, the first term is exactly $-A_{2}$ defined in Lemma 5.8. Hence, the previous equality contradicts the assumption ( $i$ i) of the theorem. The proof is thereby completed.

Proof of Theorem 1.1 We first observe that, under the assumption of the theorem, if $A_{1} \neq 1$ or respectively $A_{1}=1$ and $B_{1} \neq-k$, where $\#\left(\mathcal{K}_{b}^{+} \cup \mathcal{K}_{b}^{0,-}\right)=2 k+1$, the existence of at least one solution to Problem $(\mathcal{P})$ follows from Theorem 1.4, respectively Theorem 1.3. Hence we will assume that $A_{1}=1$ and $B_{1}=-k$ and notice that

$$
\#\left(\mathcal{K}_{i n}^{-}\right)=2 r+k, \text { where } r \in \mathbb{N}_{0},
$$

and there are $r$ even numbers $\iota\left(y_{j}\right)$ 's and $r+k$ odd numbers $\iota\left(y_{j}\right)$ 's.

Next arguing as in the proof of Theorem 1.4 using the assumption on $K_{\max } / K_{\min }$ and Proposition 4.2, we deduce that $J_{K}^{C_{\max }^{3, \infty}+\gamma}$ and $J_{K}^{C_{\max }^{4, \infty}+\gamma}$ are contractible sets. Using Corollary 3.18 , we derive that the critical points at infinity whose level are lying between these values are:

- $\left(z_{i}, z_{j}, z_{r}, z_{t}\right)_{\infty}$ with different $z_{i}$ 's which belong to $\mathcal{K}_{b}^{+} \cup \mathcal{K}_{b}^{0,-}$,

- $\left(z_{i}, z_{j}, y\right)_{\infty}$ with $y \in \mathcal{K}_{i n}^{-}$and $z_{i} \neq z_{j} \in \mathcal{K}_{b}^{+} \cup \mathcal{K}_{b}^{0,-}$,

- $\left(y_{i}, y_{j}\right)_{\infty}$ with $y_{i} \neq y_{j} \in \mathcal{K}_{i n}^{-}$.

Hence arguing as above we derive that

$$
\begin{aligned}
& \sum_{z_{i} \neq z_{j} \neq z_{r} \neq z_{t} \in \mathcal{K}_{b}^{+} \cup \mathcal{K}_{b}^{0,-}}(-1)^{3+\imath\left(z_{i}\right)+\iota\left(z_{j}\right)+\iota\left(z_{r}\right)+\iota\left(z_{t}\right)} \\
+ & \sum_{y \in \mathcal{K}_{i n}^{-} ; z_{i} \neq z_{j} \in \mathcal{K}_{b}^{+} \cup \mathcal{K}_{b}^{0,-}}(-1)^{2+\iota\left(z_{i}\right)+\iota\left(z_{j}\right)+\iota(y)}+\sum_{y_{i} \neq y_{j} \in \mathcal{K}_{i n}^{-}}(-1)^{1+\iota\left(y_{i}\right)+\iota\left(y_{j}\right)}=0 .
\end{aligned}
$$

Observe that, the first term is exactly $-A_{4}$, the second one is $A_{2} \times B_{1}$ and the third one is $-B_{2}$ (defined in Lemmas 5.8 and 5.9). Using the values of these terms (given in Lemmas 5.8 and 5.9), we obtain that

$$
r+k=0
$$

which implies that $r=k=0$. Now, from $r=k=0$, we get $\#\left(\mathcal{K}_{b}^{+} \cup \mathcal{K}_{b}^{0,-}\right)=1$ and $\# \mathcal{K}_{i n}^{-}=0$. This leads to a contradiction with the assumption that $\#\left(\mathcal{K}_{b}^{+} \cup \mathcal{K}_{b}^{0,-} \cup \mathcal{K}_{i n}^{-}\right) \geq 2$. Thereby the proof of the theorem is completed. 
Funding Open Access funding enabled and organized by Projekt DEAL.

Open Access This article is licensed under a Creative Commons Attribution 4.0 International License, which permits use, sharing, adaptation, distribution and reproduction in any medium or format, as long as you give appropriate credit to the original author(s) and the source, provide a link to the Creative Commons licence, and indicate if changes were made. The images or other third party material in this article are included in the article's Creative Commons licence, unless indicated otherwise in a credit line to the material. If material is not included in the article's Creative Commons licence and your intended use is not permitted by statutory regulation or exceeds the permitted use, you will need to obtain permission directly from the copyright holder. To view a copy of this licence, visit http://creativecommons.org/licenses/by/4.0/.

\section{Appendix}

\subsection{Bubble estimates}

Lemma 5.1 For $a \in \partial \mathbb{S}_{+}^{n}$, we have $\partial \delta_{a, \lambda} / \partial v=0$ and therefore $\varphi_{a, \lambda}=\delta_{a, \lambda}$. For $a \notin \partial \mathbb{S}_{+}^{n}$, we have

(i) $\delta_{a, \lambda} \leq \varphi_{a, \lambda} \leq 2 \delta_{a, \lambda} ; \quad\left|\lambda \partial \varphi_{a, \lambda} / \partial \lambda\right| \leq c \delta_{a, \lambda} ; \quad\left|(1 / \lambda) \partial \varphi_{a, \lambda} / \partial a^{k}\right| \leq c \delta_{a, \lambda}$,

where $a^{k}$ denotes the $k$-th component of $a$.

(ii) $\varphi_{a, \lambda}=\delta_{a, \lambda}+c_{0} \frac{H(a, .)}{\lambda^{(n-2) / 2}}+f_{a, \lambda} \quad$ where

$\left|f_{a, \lambda}\right|_{\infty} \leq \frac{c}{\lambda^{(n+2) / 2} d_{a}^{n}} ; \quad\left|\lambda \frac{\partial f_{a, \lambda}}{\partial \lambda}\right|_{\infty} \leq \frac{c}{\lambda^{(n+2) / 2} d_{a}^{n}} \quad$ and $\quad\left|\frac{1}{\lambda} \frac{\partial f_{a, \lambda}}{\partial a^{k}}\right|_{\infty} \leq \frac{c}{\lambda^{(n+4) / 2} d_{a}^{n+1}}$,

where $d_{a}:=d\left(a, \partial \mathbb{S}_{+}^{n}\right)$.

Proof Using a stereographic projection, we are led to prove the corresponding estimates on $\mathbb{R}_{+}^{n}$. We still denote by $G$ and $H$ the Green's function and its regular part of Laplacian on $\mathbb{R}_{+}^{n}$ under Neumann boundary conditions. In this case, we have

$$
\delta_{a, \lambda}(x):=c_{0} \frac{\lambda^{(n-2) / 2}}{\left(1+\lambda^{2}|x-a|^{2}\right)^{(n-2) / 2}} \quad \text { and } \quad H(a, x):=\frac{1}{|x-\bar{a}|^{n-2}},
$$

where $\bar{a}$ denotes the symmetric point of $a$ with respect to $\partial \mathbb{R}_{+}^{n}$. Let $\psi:=\delta_{a, \lambda}+\delta_{\bar{a}, \lambda}$. Easy computation implies that $\partial \psi / \partial v=0$.

To prove the first inequality, let us consider $h:=\varphi_{a, \lambda}-\delta_{a, \lambda}$. Hence we get $\Delta h=0$ and $\partial h / \partial v=-\partial \delta_{a, \lambda} / \partial v>0$. Hence, using the Green's representation, we derive that $h>0$ in $\mathbb{R}_{+}^{n}$.

For the second inequality, let us consider $h:=\psi-\varphi_{a, \lambda}$. Easy computations imply that $\partial h / \partial v=0$ and $-\Delta h=-\Delta \delta_{\bar{a}, \lambda}>0$. Hence, $h>0$ in $\mathbb{R}_{+}^{n}$. The inequality follows from the fact that $\delta_{\bar{a}, \lambda} \leq \delta_{a, \lambda}$ in $\mathbb{R}_{+}^{n}$.

For the third one, let $g:=\lambda \partial \varphi_{a, \lambda} / \partial \lambda$, observe that $\partial g / \partial v=0$ and $|\Delta g| \leq((n+$ $2) / 2) \delta_{a, \lambda}^{(n+2) /(n-2)}$. Now let us consider $h:=((n+2) / 2) \psi \pm g$. It follows that $-\Delta h>0$ and $\partial h / \partial v=0$. Hence $h>0$ in $\mathbb{R}_{+}^{n}$ which gives the proof of the third inequality. The fourth one follows by the same way. 
Concerning the second claim, it is easy to see that $\Delta f_{a, \lambda}=0$ and

$$
\begin{aligned}
\frac{\partial f_{a, \lambda}}{\partial v} & =-\frac{\partial \delta_{a, \lambda}}{\partial v}-\frac{c_{0}}{\lambda^{(n-2) / 2}} \frac{\partial H(a, .)}{\partial v}=c_{0}(n-2) \frac{\lambda^{(n+2) / 2} d_{a}}{\left(1+\lambda^{2}|x-a|^{2}\right)^{n / 2}}-\frac{c_{0}}{\lambda^{(n-2) / 2}} \frac{(n-2) d_{a}}{|x-\bar{a}|^{n}} \\
& =O\left(\frac{d_{a}}{\lambda^{(n+2) / 2}|x-a|^{n+2}}\right) .
\end{aligned}
$$

Now, using the Green's representation, we get

$$
\begin{aligned}
\left|f_{a, \lambda}(x)\right| & \leq c \int_{\partial \mathbb{R}_{+}^{n}} G(x, y)\left|\frac{\partial f_{a, \lambda}}{\partial v}(y)\right| d y \leq \frac{c d_{a}}{\lambda^{(n+2) / 2}} \int_{\partial \mathbb{R}_{+}^{n}} G(x, y) \frac{1}{|y-\bar{a}|^{n+2}} d y \\
& \leq \frac{c}{\lambda^{(n+2) / 2} d_{a}} \int_{\partial \mathbb{R}_{+}^{n}} G(x, y) \frac{1}{|y-\bar{a}|^{n}} d y \leq \frac{c}{\lambda^{(n+2) / 2} d_{a}} \frac{H(a, x)}{d_{a}} \leq \frac{c}{\lambda^{(n+2) / 2} d_{a}^{n}} .
\end{aligned}
$$

This gives the first claim in ( $i$ i $)$. The other ones can be done by the same way.

Lemma 5.2 1) For each $i \neq j$, we have

$$
-\lambda_{i} \frac{\partial \varepsilon_{i j}}{\partial \lambda_{i}}-\lambda_{j} \frac{\partial \varepsilon_{i j}}{\partial \lambda_{j}} \geq 0 \text { and }-\lambda_{i} \frac{\partial \varepsilon_{i j}}{\partial \lambda_{i}} \geq c \varepsilon_{i j} \text { if } \lambda_{i} \geq c \lambda_{j} \text { or } \lambda_{i} d\left(a_{i}, a_{j}\right) \geq 2 \text {. }
$$

2) Let $i, j \in I_{b}:=\left\{k: a_{k} \in \partial \mathbb{S}_{+}^{n}\right\}$ and let $\mu_{i}$ and $\mu_{j}$ be defined by (15). Assume that $\mu_{j} \leq c^{\prime} \mu_{i}$ for some constant $c^{\prime}$, then: (i) either there exists a constant $c^{\prime \prime}$ such that $\lambda_{j} \leq c^{\prime \prime} \lambda_{i}$, (ii) or $\lambda_{i} d\left(a_{i}, a_{j}\right) \geq 2$.

Proof The proof of the first assertion follows immediately from the definition of $\varepsilon_{i j}$. Concerning the second one, observe that, if $\left|\nabla K\left(a_{i}\right)\right| \geq c$ and $\left|\nabla K\left(a_{j}\right)\right| \geq c$, then it follows that $\mu_{k}$ and $\lambda_{k}$ are of the same order (that is: the ratio is bounded from above and below) for $k=i, j$. Hence the result follows in this case. In the other case, there exists $k \in\{i, j\}$ such that $a_{k}$ is close to a critical point $z$ of $K$ in $\partial \mathbb{S}_{+}^{n}$ (i.e. $\partial K / \partial v(z)=0$ ). Arguing by contradiction, assume that $\lambda_{i} d\left(a_{i}, a_{j}\right) \leq 2$ and $\lambda_{j} / \lambda_{i}$ is very large. It follows that $a_{i}$ and $a_{j}$ are close to the same critical point $z$. Now we claim that:

Claim 1: $\lambda_{j}\left|\nabla K\left(a_{j}\right)\right|$ is very large.

In fact, if it is not, we derive that $\left|\nabla K\left(a_{j}\right)\right| / \lambda_{j} \leq c / \lambda_{j}^{2}$ which implies that $1 / \mu_{j} \leq c / \lambda_{j}^{2}$ and therefore $1 / \mu_{j}$ is very small with respect to $1 / \lambda_{i}^{2} \leq 1 / \mu_{i}$. This gives a contradiction and therefore our claim follows.

Since $z$ is a non degenerate critical point of $K_{1}$, it follows that $\lambda_{j} d\left(a_{j}, z\right)$ is very large. Moreover, Claim 1 implies that $\left|\nabla K\left(a_{j}\right)\right| / \lambda_{j} \leq 1 / \mu_{j} \leq c\left|\nabla K\left(a_{j}\right)\right| / \lambda_{j}$. Now we claim that:

Claim 2: $\lambda_{i}\left|\nabla K\left(a_{i}\right)\right| \geq 1$ cannot occur.

To prove this claim, we assume that the inequality is true. Then we derive that $\left|\nabla K\left(a_{i}\right)\right| / \lambda_{i} \leq 1 / \mu_{i} \leq 2\left|\nabla K\left(a_{i}\right)\right| / \lambda_{i}$. Since $\mu_{j} \leq c^{\prime} \mu_{i}$, we derive that $\left|\nabla K\left(a_{i}\right)\right| / \lambda_{i} \leq$ $c\left|\nabla K\left(a_{j}\right)\right| / \lambda_{j}$ and therefore $\lambda_{j} d\left(a_{i}, z\right) \leq c \lambda_{i} d\left(a_{j}, z\right)$ which implies that $d\left(a_{i}, z\right)$ is very small with respect to $d\left(a_{i}, z\right)$ and therefore $d\left(a_{i}, z\right)$ is very small with respect to $d\left(a_{i}, a_{j}\right)$. Now observe that, since we assumed that $\lambda_{i}\left|\nabla K\left(a_{i}\right)\right| \geq 1$, it follows that $\lambda_{i} d\left(a_{i}, z\right) \geq c$ and therefore $\lambda_{i} d\left(a_{i}, a_{j}\right)$ becomes very large which gives a contradiction. Hence Claim 2 follows.

Finally, we claim that

Claim 3: $\lambda_{i}\left|\nabla K\left(a_{i}\right)\right| \leq 1$ cannot occur.

Arguing by contradiction we assume that $\lambda_{i} d\left(a_{i}, z\right) \leq c$. From $\mu_{j} \leq c^{\prime} \mu_{i}$, we derive that $1 / \lambda_{i}^{2} \leq c\left|\nabla K\left(a_{j}\right)\right| / \lambda_{j} \leq c d\left(a_{j}, z\right) / \lambda_{j}$ and therefore $\lambda_{j} / \lambda_{i} \leq c \lambda_{i} d\left(a_{j}, z\right)$, that is $\lambda_{i} d\left(a_{j}, z\right)$ is very large. But we have $\lambda_{i} d\left(a_{j}, a_{i}\right) \leq 2$ and $\lambda_{i} d\left(a_{i}, z\right) \leq c$ which imply that $\lambda_{i} d\left(a_{j}, z\right)$ is bounded. Hence we get a contradiction which completes the proof of Claim 3 . 
Hence the lemma is fully proven.

\subsection{Asymptotic expansion of the functional and its gradient}

Proposition 5.3 Let $n \geq 5$ and $u=\sum_{i \leq q} \alpha_{i} \delta_{i}+\sum_{i>q} \alpha_{i} \varphi_{i} \in V(m, q, p, \varepsilon)$ be such that: (i) $d\left(a_{i}, a_{j}\right) \geq c>0$ for every $i \neq j$, (ii) for $i>q$, $a_{i}$ is close to a critical point $y_{j_{i}}$ of $K$ in $\mathbb{S}_{+}^{n}$ and (iii) for $i \leq q, a_{i}$ is close to a critical point $z_{j_{i}}$ of $K_{1}$ in $\partial \mathbb{S}_{+}^{n}$. Then the following expansion holds

$$
\begin{aligned}
J_{K}(u)= & \frac{\left(\sum_{i \leq q} \alpha_{i}^{2}+2 \sum_{i>q} \alpha_{i}^{2}\right) S_{n}^{2 / n}}{\left(\sum_{i \leq q} \alpha_{i}^{2 n / n-2} K\left(a_{i}\right)+2 \sum_{i>q} \alpha_{i}^{2 n / n-2} K\left(a_{i}\right)\right)^{n-2 / n}}\left(1-2 c_{6} J_{K}(u)^{\frac{n}{n-2}} \sum_{i>q} \alpha_{i}^{\frac{2 n}{n-2}} \frac{\Delta K\left(a_{i}\right)}{\lambda_{i}^{2}}\right. \\
& \left.+J_{K}(u)^{\frac{n}{n-2}} \sum_{i \leq q} \alpha_{i}^{\frac{2 n}{n-2}}\left(\frac{c_{7}}{\lambda_{i}} \frac{\partial K}{\partial v}\left(a_{i}\right)-c_{6} \frac{\Delta K\left(a_{i}\right)}{\lambda_{i}^{2}}\right)+\sum_{i=1}^{p+q} O\left(\frac{1}{\lambda_{i}^{3}}+\frac{\left|\nabla K\left(a_{i}\right)\right|^{2}}{\lambda_{i}^{2}}\right)\right)
\end{aligned}
$$

where

$$
\begin{aligned}
S_{n} & :=c_{0}^{\frac{2 n}{n-2}} \int_{\mathbb{R}_{+}^{n}} \frac{d x}{\left(1+|x|^{2}\right)^{n}} ; c_{6}:=\frac{n-2}{n^{2}} c_{0}^{\frac{2 n}{n-2}} \int_{\mathbb{R}_{+}^{n}} \frac{|x|^{2} d x}{\left(1+|x|^{2}\right)^{n}} ; \\
c_{7} & :=2 \frac{n-2}{n} c_{0}^{\frac{2 n}{n-2}} \int_{\mathbb{R}_{+}^{n}} \frac{x_{n} d x}{\left(1+|x|^{2}\right)^{n}}
\end{aligned}
$$

Proof From the definition of $J_{K}$, we need to expand (using the fact that $\bar{v} \perp \varphi_{i}$ for each $i$ )

$$
\begin{aligned}
\|u\|^{2} & =\sum \alpha_{i}^{2}\left\|\varphi_{i}\right\|^{2}+\|\bar{v}\|^{2}+O\left(\sum \varepsilon_{i j}\right) \\
& =S_{n}\left(\sum_{i \leq q} \alpha_{i}^{2}+2 \sum_{i>q} \alpha_{i}^{2}\right)+\|\bar{v}\|^{2}+O\left(\sum \varepsilon_{i j}+\sum_{i>q} \frac{1}{\lambda_{i}^{n-2}}\right), \\
\int_{\mathbb{S}_{+}^{n}} K u^{\frac{2 n}{n-2}} & =\sum_{i=1}^{q+p} \alpha_{i}^{\frac{2 n}{n-2}} \int_{\mathbb{S}_{+}^{n}} K \varphi_{i}^{\frac{2 n}{n-2}}+\frac{2 n}{n-2} \int_{\mathbb{S}_{+}^{n}} K\left(\sum \alpha_{i} \varphi_{i}\right)^{\frac{n+2}{n-2}} \bar{v} \\
& +O\left(\sum_{i \neq j} \int \varphi_{i}^{\frac{n+2}{n-2}} \varphi_{j}+\|\bar{v}\|^{2}\right) .
\end{aligned}
$$

The last integral is equal to $O\left(\varepsilon_{i j}\right)$. The second one is presented in (9). Concerning the first one, for $i>q$, using Lemma 5.1, we get

$$
\begin{aligned}
\int_{\mathbb{S}_{+}^{n}} K \varphi_{i}^{\frac{2 n}{n-2}} & =\int_{\mathbb{R}_{+}^{n}} \widetilde{K} \delta_{i}^{\frac{2 n}{n-2}}+O\left(\frac{1}{\lambda_{i}^{(n-2) / 2}} \int \delta_{i}^{\frac{n+2}{n-2}}\right) \\
& =\int_{B\left(a_{i}, d_{i}\right)} \widetilde{K} \delta_{i}^{\frac{2 n}{n-2}}+O\left(\int_{\mathbb{R}^{n} \backslash B\left(a_{i}, d_{i}\right)} \delta_{i}^{\frac{2 n}{n-2}}+\frac{1}{\lambda_{i}^{(n-2) / 2}} \int \delta_{i}^{\frac{n+2}{n-2}}\right) \\
& =2 S_{n} \widetilde{K}\left(a_{i}\right)+\frac{1}{2 n} \frac{\Delta \widetilde{K}\left(a_{i}\right)}{\lambda_{i}^{2}} c_{0}^{\frac{2 n}{n-2}} \int_{\mathbb{R}^{n}} \frac{|x|^{2}}{\left(1+|x|^{2}\right)^{n}} d x+O\left(\frac{1}{\lambda_{i}^{3}}\right)
\end{aligned}
$$


However, for $i \leq q$, we have $\varphi_{i}=\delta_{i}$ and therefore

$$
\begin{aligned}
\int_{\mathbb{S}_{+}^{n}} K \delta_{i}^{\frac{2 n}{n-2}}= & \int_{\mathbb{R}_{+}^{n}} \widetilde{K} \delta_{i}^{\frac{2 n}{n-2}}=S_{n} \widetilde{K}\left(a_{i}\right)+\nabla \widetilde{K}\left(a_{i}\right) c_{0}^{\frac{2 n}{n-2}} \int_{\mathbb{R}_{+}^{n}} \frac{\lambda_{i}^{n}\left(x-a_{i}\right)}{\left(1+\lambda_{i}^{2}\left|x-a_{i}\right|^{2}\right)^{n}} d x \\
& +\frac{1}{2} \sum \frac{\partial^{2} \widetilde{K}\left(a_{i}\right)}{\partial x_{k} \partial x_{\ell}} c_{0}^{\frac{2 n}{n-2}} \int_{\mathbb{R}_{+}^{n}} \frac{\lambda_{i}^{n}\left(x-a_{i}\right)_{k}\left(x-a_{i}\right)_{\ell}}{\left(1+\lambda_{i}^{2}\left|x-a_{i}\right|^{2}\right)^{n}} d x+O\left(\frac{1}{\lambda_{i}^{3}}\right) \\
= & S_{n} \widetilde{K}\left(a_{i}\right)-\frac{\partial \widetilde{K}}{\partial v}\left(a_{i}\right) c_{0}^{\frac{2 n}{n-2}} \int_{\mathbb{R}_{+}^{n}} \frac{x_{n}}{\left(1+|x|^{2}\right)^{n}} d x \\
& +\frac{1}{2 n} \frac{\Delta \widetilde{K}\left(a_{i}\right)}{\lambda_{i}^{2}} c_{0}^{\frac{2 n}{n-2}} \int_{\mathbb{R}_{+}^{n}} \frac{|x|^{2}}{\left(1+|x|^{2}\right)^{n}} d x+O\left(\frac{1}{\lambda_{i}^{3}}\right) .
\end{aligned}
$$

Note that, since $u \in \Sigma$, we deduce that

$$
J_{K}(u)=\frac{1}{\Gamma^{\frac{n-2}{n}}}\left(1+O\left(\sum_{i>q} \frac{1}{\lambda_{i}^{2}}+\sum_{i \leq q} \frac{1}{\lambda_{i}}\left|\frac{\partial K}{\partial v}\left(a_{i}\right)\right|+\frac{1}{\lambda_{i}^{2}}+\|\bar{v}\|^{2}\right)\right)
$$

where

$$
\Gamma:=\sum_{i \leq q} \alpha_{i}^{\frac{2 n}{n-2}} K\left(a_{i}\right)+2 \sum_{i>q} \alpha_{i}^{\frac{2 n}{n-2}} K\left(a_{i}\right) .
$$

Now, the precise expansion of $J_{K}$ follows from the above estimates, the estimate of $\|\bar{v}\|$ (see Lemma 2.3) and the fact that $(1+x)^{-(n-2) / n}=1-((n-2) / n) x+O\left(x^{2}\right)$.

In the following, we will present the expansion of the gradient of $J_{K}$ in the potential sets. We will present the results for $p+q \geq 2$. However, the results are true for $p+q=1$, it suffices to remove the terms $\varepsilon_{i j}$ 's which correspond to the interaction terms of the bubbles.

Proposition 5.4 Let $n \geq 5$, for $u=\sum_{i \leq q} \alpha_{i} \delta_{i}+\sum_{i>q} \alpha_{j} \varphi_{j} \in V(m, q, p, \varepsilon)$ and $i \leq q$, it holds

$$
\begin{aligned}
& \left\langle\nabla J_{K}(u), \lambda_{i} \frac{\partial \delta_{i}}{\partial \lambda_{i}}\right\rangle=2 J_{K}(u)\left[-\frac{c_{2}}{2} \sum_{j \neq i ; j \leq q} \alpha_{j} \lambda_{i} \frac{\partial \varepsilon_{i j}}{\partial \lambda_{i}}(1+o(1))\right. \\
& \left.+2 J_{K}(u)^{\frac{n}{n-2}} \alpha_{i}^{\frac{n+2}{n-2}}\left(-\frac{c_{3}}{\lambda_{i}} \frac{\partial K}{\partial v}\left(a_{i}\right)+c_{9} \frac{\Delta K\left(a_{i}\right)}{\lambda_{i}^{2}}\right)\right]+O\left(\frac{1}{\lambda_{i}^{3}}+\sum_{j>q} \varepsilon_{i j}+R_{1}^{b}\right)
\end{aligned}
$$

where

$$
R_{1}^{b}:=\sum_{k \leq q}\left(\frac{\left|\nabla K\left(a_{k}\right)\right|}{\lambda_{k}}\right)^{\frac{n}{2}}+\left(\frac{1}{\lambda_{k}^{2}}\right)^{\frac{n+1}{3}}+\sum_{j \neq k ; j, k \leq q} \varepsilon_{k j}^{\frac{n}{n-2}} \ln \left(\varepsilon_{k j}^{-1}\right) ; \quad c_{3}=\frac{n-2}{2} c_{0}^{\frac{2 n}{n-2}} \int_{\mathbb{R}_{+}^{n}} \frac{x_{n}\left(|x|^{2}-1\right)}{\left(1+|x|^{2}\right)^{n+1}} d x .
$$

Proof

$\left\langle\nabla J_{K}(u), \lambda_{i} \frac{\partial \delta_{i}}{\partial \lambda_{i}}\right\rangle=2 J_{K}(u)\left(\sum_{j \leq q} \alpha_{j}\left\langle\delta_{j}, \lambda_{i} \frac{\partial \delta_{i}}{\partial \lambda_{i}}\right\rangle-J(u)^{n /(n-2)} \int K\left(\sum_{j \leq q} \alpha_{j} \delta_{j}\right)^{\frac{n+2}{n-2}} \lambda_{i} \frac{\partial \delta_{i}}{\partial \lambda_{i}}+\sum_{j>q} O\left(\varepsilon_{i j}\right)\right)$.

For $j \leq q$, we have $a_{j} \in \partial \mathbb{S}_{+}^{n}$ and therefore, using [5], we get, for $j \neq i$,

$$
\begin{aligned}
& \left\langle\delta_{j}, \lambda_{i} \frac{\partial \delta_{i}}{\partial \lambda_{i}}\right\rangle=\int_{\mathbb{R}_{+}^{n}} \delta_{j}^{\frac{n+2}{n-2}} \lambda_{i} \frac{\partial \delta_{i}}{\partial \lambda_{i}}=\frac{1}{2} \int_{\mathbb{R}^{n}} \delta_{j}^{\frac{n+2}{n-2}} \lambda_{i} \frac{\partial \delta_{i}}{\partial \lambda_{i}}=\frac{1}{2} c_{2} \varepsilon_{i j}+O\left(\varepsilon_{i j}^{\frac{n}{n-2}} \ln \left(\varepsilon_{i j}^{-1}\right)\right) \\
& \left\langle\delta_{i}, \lambda_{i} \frac{\partial \delta_{i}}{\partial \lambda_{i}}\right\rangle=\int_{\mathbb{R}_{+}^{n}} \delta_{i}^{\frac{n+2}{n-2}} \lambda_{i} \frac{\partial \delta_{i}}{\partial \lambda_{i}}=\frac{1}{2} \int_{\mathbb{R}^{n}} \delta_{i}^{\frac{n+2}{n-2}} \lambda_{i} \frac{\partial \delta_{i}}{\partial \lambda_{i}}=0 .
\end{aligned}
$$


Concerning the other term, it holds

$$
\begin{aligned}
\int & K\left(\sum_{j \leq q} \alpha_{j} \delta_{j}\right)^{\frac{n+2}{n-2}} \lambda_{i} \frac{\partial \delta_{i}}{\partial \lambda_{i}}=\sum_{j \leq q} \int K\left(\alpha_{j} \delta_{j}\right)^{\frac{n+2}{n-2}} \lambda_{i} \frac{\partial \delta_{i}}{\partial \lambda_{i}} \\
+ & \frac{n+2}{n-2} \int K\left(\alpha_{i} \delta_{i}\right)^{\frac{4}{n-2}}\left(\sum_{j \leq q ; j \neq i} \alpha_{j} \delta_{j}\right) \lambda_{i} \frac{\partial \delta_{i}}{\partial \lambda_{i}}+O\left(\sum_{k \neq r} \int\left(\delta_{k} \delta_{r}\right)^{\frac{n}{n-2}}\right) .
\end{aligned}
$$

Observe that, for $j \neq i$, expanding $K$ around $a_{j}$, we get

$$
\begin{aligned}
& \int_{\mathbb{R}_{+}^{n}} K \delta_{j}^{\frac{n+2}{n-2}} \lambda_{i} \frac{\partial \delta_{i}}{\partial \lambda_{i}} \\
& =K\left(a_{j}\right) \int_{\mathbb{R}_{+}^{n}} \delta_{j}^{\frac{n+2}{n-2}} \lambda_{i} \frac{\partial \delta_{i}}{\partial \lambda_{i}}+O\left(\left|\nabla K\left(a_{j}\right)\right| \int_{\mathbb{R}_{+}^{n}}\left|x-a_{j}\right| \delta_{j}^{\frac{n+2}{n-2}} \delta_{i}+\int_{\mathbb{R}_{+}^{n}}\left|x-a_{j}\right|^{2} \delta_{j}^{\frac{n+2}{n-2}} \delta_{i}\right) \\
& =K\left(a_{j}\right) \frac{1}{2} c_{2} \varepsilon_{i j}+O\left(\varepsilon_{i j}^{\frac{n}{n-2}} \ln \left(\varepsilon_{i j}^{-1}\right)+\frac{\left|\nabla K\left(a_{j}\right)\right|}{\lambda_{j}} \varepsilon_{i j}\left(\ln \varepsilon_{i j}^{-1}\right)^{\frac{n-2}{n}}+\frac{1}{\lambda_{j}^{2}} \varepsilon_{i j}^{\frac{n}{n+1}}\left(\ln \varepsilon_{i j}^{-1}\right)^{\frac{n-2}{n+1}}\right) \\
& =K\left(a_{j}\right) \frac{1}{2} c_{2} \varepsilon_{i j}+O\left(\varepsilon_{i j}^{\frac{n}{n-2}} \ln \left(\varepsilon_{i j}^{-1}\right)+\left(\frac{\left|\nabla K\left(a_{j}\right)\right|}{\lambda_{j}}\right)^{n / 2}+\left(\frac{1}{\lambda_{j}^{2}}\right)^{(n+1) / 3}\right), \\
& \int_{\mathbb{R}_{+}^{n}} K \delta_{i}^{\frac{n+2}{n-2}} \lambda_{i} \frac{\partial \delta_{i}}{\partial \lambda_{i}}=\sum_{k} \frac{\partial K}{\partial x_{k}}\left(a_{i}\right) \int_{\mathbb{R}_{+}^{n}}\left(x-a_{i}\right)_{k} \delta_{i}^{\frac{n+2}{n-2}} \lambda_{i} \frac{\partial \delta_{i}}{\partial \lambda_{i}} \\
& +\frac{1}{2} \sum \frac{\partial^{2} K}{\partial x_{k} \partial x_{\ell}}\left(a_{i}\right) \int_{\mathbb{R}_{+}^{n}}\left(x-a_{i}\right)_{k}\left(x-a_{i}\right)_{\ell} \delta_{i}^{\frac{n+2}{n-2}} \lambda_{i} \frac{\partial \delta_{i}}{\partial \lambda_{i}}+O\left(\int_{\mathbb{R}_{+}^{n}}\left|x-a_{i}\right|^{3} \delta_{i}^{\frac{2 n}{n-2}}\right) \\
& =\frac{c_{3}}{\lambda_{i}} \frac{\partial K}{\partial v}\left(a_{i}\right)-c_{9} \frac{\Delta K\left(a_{i}\right)}{\lambda_{i}^{2}}+O\left(\frac{1}{\lambda_{i}^{3}}\right) .
\end{aligned}
$$

Finally, for $j \neq i$, it holds

$$
\begin{aligned}
\frac{n+2}{n-2} \int_{\mathbb{R}_{+}^{n}} K \delta_{i}^{\frac{4}{n-2}} \delta_{j} \lambda_{i} \frac{\partial \delta_{i}}{\partial \lambda_{i}} & =K\left(a_{i}\right)\left\langle\delta_{j}, \lambda_{i} \frac{\partial \delta_{i}}{\partial \lambda_{i}}\right\rangle+O\left(\left|\nabla K\left(a_{i}\right)\right| \int_{\mathbb{R}_{+}^{n}}\left|x-a_{i}\right| \delta_{i}^{\frac{n+2}{n-2}} \delta_{j}\right. \\
& \left.+\int_{\mathbb{R}_{+}^{n}}\left|x-a_{i}\right|^{2} \delta_{i}^{\frac{n+2}{n-2}} \delta_{j}\right) .
\end{aligned}
$$

Hence the proof follows.

Proposition 5.5 Let $n \geq 5$. For $u=\sum_{i \leq q} \alpha_{i} \delta_{i}+\sum_{i>q} \alpha_{j} \varphi_{j} \in V(m, q, p, \varepsilon)$ and $i \leq q$, it holds:

$$
\begin{aligned}
& \left\langle\nabla J_{K}(u), \frac{1}{\lambda_{i}} \frac{\partial \delta_{i}}{\partial a_{i}}\right\rangle=2 J_{K}(u) \alpha_{i} e_{n}\left[c_{4}\left(1-J_{K}(u)^{\frac{n}{n-2}} \alpha_{i}^{\frac{4}{n-2}} K\left(a_{i}\right)\right)+J_{K}(u)^{\frac{n}{n-2}} \alpha_{i}^{\frac{4}{n-2}} \frac{c_{5}}{\lambda_{i}} \frac{\partial K}{\partial v}\left(a_{i}\right)\right] \\
& -J_{K}(u) c_{2} \sum_{j \leq q ; j \neq i} \alpha_{j} \frac{1}{\lambda_{i}} \frac{\partial \varepsilon_{i j}}{\partial a_{i}}\left(-1+J_{K}(u)^{\frac{n}{n-2}} \sum_{k=i, j} \alpha_{k}^{\frac{4}{n-2}} K\left(a_{k}\right)\right)+O\left(\frac{1}{\lambda_{i}^{2}}\right) \\
& -4 J_{K}(u)^{\frac{2(n-1)}{n-2}} \alpha_{i}^{\frac{n+2}{n-2}} \frac{2 c_{5}}{\lambda_{i}} \nabla_{T} K\left(a_{i}\right)+O\left(R_{1}^{b}+\sum_{k \leq q ; k \neq i} \varepsilon_{i k}^{\frac{n+1}{n-2}} \lambda_{k} d\left(a_{i}, a_{k}\right)+\sum_{k>q} \varepsilon_{i k}\right)
\end{aligned}
$$

where $R_{1}^{b}$ is defined in Proposition 5.4 and

$$
c_{4}=(n-2) c_{0}^{\frac{2 n}{n-2}} \int_{\mathbb{R}_{+}^{n}} \frac{x_{n}}{\left(1+|x|^{2}\right)^{n+1}} d x \quad \text { and } c_{5}=\frac{n-2}{2 n} c_{0}^{\frac{2 n}{n-2}} \int_{\mathbb{R}^{n}} \frac{x_{n}^{2}}{\left(1+|x|^{2}\right)^{n+1}} d x .
$$


Proof The proof can be done as the previous one.

Proposition 5.6 For $u=\sum \alpha_{j} \varphi_{j} \in V(m, q, p, \varepsilon)$ and $i \leq q$, we have the following expansion:

$\left\langle\nabla J_{K}(u), \delta_{i}\right\rangle=2 J_{K}(u) \alpha_{i} S_{n}\left(1-J_{K}(u)^{\frac{n}{n-2}} \alpha_{i}^{\frac{4}{n-2}} K\left(a_{i}\right)\right)+O\left(\frac{\left|\nabla K\left(a_{i}\right)\right|}{\lambda_{i}}+\frac{1}{\lambda_{i}^{2}}+\sum_{j \neq i} \varepsilon_{i j}\right)$. where $S_{n}$ is defined in Proposition 5.3.

Proof

$$
\left\langle\nabla J_{K}(u), \delta_{i}\right\rangle=2 J_{K}(u) \alpha_{i}\left\|\delta_{i}\right\|^{2}-J_{K}(u)^{n /(n-2)} \int K \delta_{i}^{\frac{2 n}{n-2}}+O\left(\sum \varepsilon_{k i}\right) .
$$

Observe that

$$
\int K \delta_{i}^{\frac{2 n}{n-2}}=K\left(a_{i}\right) \int \delta_{i}^{\frac{2 n}{n-2}}+O\left(\left|\nabla K\left(a_{i}\right)\right| \int\left|x-a_{i}\right| \delta_{i}^{\frac{2 n}{n-2}}+\int\left|x-a_{i}\right|^{2} \delta_{i}^{\frac{2 n}{n-2}}\right)
$$

which gives the result.

Proposition 5.7 For $u=\sum_{j \leq q} \alpha_{j} \delta_{j}+\sum_{j>q} \alpha_{j} \varphi_{j} \in V(m, q, p, \varepsilon)$ and for each $i \geq q+1$, we have:

$$
\begin{aligned}
&\left\langle\nabla J_{K}(u), \lambda_{i} \frac{\partial \varphi_{i}}{\partial \lambda_{i}}\right\rangle=2 J_{K}\left(-c_{2} \sum_{j \neq i} \alpha_{j}(1+o(1)) \lambda_{i} \frac{\partial \varepsilon_{i j}}{\partial \lambda_{i}}+c_{2} \frac{n-2}{2} \sum_{j=q+1}^{p} \alpha_{j}(1+o(1)) \frac{H\left(a_{i}, a_{j}\right)}{\left(\lambda_{i} \lambda_{j}\right)^{(n-2) / 2}}\right. \\
&\left.+c \alpha_{i}(1+o(1)) \frac{\Delta K\left(a_{i}\right)}{\lambda_{i}^{2} K\left(a_{i}\right)}\right)+O\left(\frac{1}{\lambda_{i}^{3}}+R_{1}\right), \\
&\left\langle\nabla J_{K}(u), \varphi_{i}\right\rangle=2 J_{K}(u) \alpha_{i} S_{n}\left(1-J_{K}(u)^{\frac{n}{n-2}} \alpha_{i}^{\frac{4}{n-2}} K\left(a_{i}\right)\right) \\
&+O\left(\frac{\left|\nabla K\left(a_{i}\right)\right|}{\lambda_{i}}+\frac{1}{\lambda_{i}^{2}}+\frac{1}{\left(\lambda_{i} d_{i}\right)^{n-2}}+\sum_{j \neq i} \varepsilon_{i j}\right), \\
&\left\langle\nabla J_{K}(u), \frac{1}{\lambda_{i}} \frac{\partial \varphi_{i}}{\partial a_{i}}\right\rangle \cdot \nabla K\left(a_{i}\right) \geq c \frac{\left|\nabla K\left(a_{i}\right)\right|^{2}}{\lambda_{i}}+O\left(\left(\frac{1}{\lambda_{i}^{2}}+\frac{1}{\left(\lambda_{i} d_{i}\right)^{n-2}}+\sum_{j \neq i} \varepsilon_{i j}\right)\left|\nabla K\left(a_{i}\right)\right|\right)
\end{aligned}
$$

where

$$
R_{1}:=\sum_{k=1}^{q+p}\left(\frac{\left|\nabla K\left(a_{k}\right)\right|}{\lambda_{k}}\right)^{\frac{n}{2}}+\left(\frac{1}{\lambda_{k}^{2}}\right)^{\frac{n+1}{3}}+\sum_{j \neq k} \varepsilon_{k j}^{\frac{n}{n-2}} \ln \left(\varepsilon_{k j}^{-1}\right)+\sum_{j>q} \frac{1}{\left(\lambda_{j} d_{j}\right)^{n}} .
$$

\subsection{Counting index formulae}

Lemma 5.8 Let $z_{1}, \ldots, z_{N}$ be $N$ critical points of $K_{1}$ in $\partial \mathbb{S}_{+}^{n}$ and let $\iota\left(z_{j}\right):=n-1-$ $\operatorname{morse}\left(K_{1}, z_{j}\right)$. Assume that

$$
A_{1}:=\sum_{j=1}^{N}(-1)^{i\left(z_{j}\right)}=1
$$


Then the number $N$ has to be odd, say $N:=2 k+1$ (with $k \in \mathbb{N}_{0}$ ) and there are $k$ odd numbers $\iota\left(z_{j}\right)$ 's and $k+1$ even numbers $\iota\left(z_{j}\right)$ 's. Furthermore, for each $k \geq 0$, it hold

$$
\begin{aligned}
A_{2} & :=\sum_{j<\ell}(-1)^{\iota\left(z_{j}\right)+\iota\left(z_{\ell}\right)}=-k ; \quad A_{3}:=\sum_{j<\ell<r}(-1)^{\iota\left(z_{j}\right)+\iota\left(z_{\ell}\right)+\iota\left(z_{r}\right)}=-k, \\
A_{4} & :=\sum_{j<\ell<r<t}(-1)^{\iota\left(z_{j}\right)+\iota\left(z_{\ell}\right)+\iota\left(z_{r}\right)+\iota\left(z_{t}\right)}=\frac{1}{2} k(k-1) .
\end{aligned}
$$

Proof To compute the value of $A_{2}$, observe that it is the sum of +1 and -1 . To get $-1, \iota\left(z_{j}\right)$ and $\iota\left(z_{k}\right)$ have to be of different parity. However, to get $+1, \iota\left(z_{j}\right)$ and $\iota\left(z_{k}\right)$ have to be of the same parity. A similar argument holds for the computation of the values $A_{3}$ and $A_{4}$. Hence:

- For $k=0$, we have only one point $z$ with an even $\iota(z)$. Thus $A_{2}=A_{3}=A_{4}=0$.

- For $k=1$, we have two points $z_{0}$ and $z_{2}$ with even $\iota\left(z_{k}\right)$ and one point $z_{1}$ with an odd $\iota\left(z_{1}\right)$. Thus, $A_{4}=0, A_{3}=-1$ and $A_{2}=1-2=-1$.

- For $k \geq 2$, there exist $k+1$ even numbers $\iota\left(z_{j}\right)$ and $k$ odd numbers $\iota\left(z_{j}\right)$. Thus, it holds

$$
\begin{aligned}
A_{2} & =\left(\begin{array}{c}
2 \\
k+1
\end{array}\right)+\left(\begin{array}{l}
2 \\
k
\end{array}\right)-\left(\begin{array}{c}
1 \\
k+1
\end{array}\right)\left(\begin{array}{l}
1 \\
k
\end{array}\right)=\frac{1}{2}(k+1) k+\frac{1}{2} k(k-1)-(k+1) k=-k, \\
A_{3} & =\left(\begin{array}{l}
3 \\
3
\end{array}\right)+\left(\begin{array}{l}
1 \\
3
\end{array}\right)\left(\begin{array}{l}
2 \\
2
\end{array}\right)-\left(\begin{array}{l}
2 \\
3
\end{array}\right)\left(\begin{array}{l}
1 \\
2
\end{array}\right)=-2 \quad \text { if } k=2 \\
A_{3} & =\left(\begin{array}{c}
3 \\
k+1
\end{array}\right)+\left(\begin{array}{c}
1 \\
k+1
\end{array}\right)\left(\begin{array}{l}
2 \\
k
\end{array}\right)-\left(\begin{array}{c}
2 \\
k+1
\end{array}\right)\left(\begin{array}{l}
1 \\
k
\end{array}\right)-\left(\begin{array}{l}
3 \\
k
\end{array}\right)=-k \quad \text { if } k \geq 3 \\
A_{4} & =\left(\begin{array}{l}
2 \\
3
\end{array}\right)\left(\begin{array}{l}
2 \\
2
\end{array}\right)-\left(\begin{array}{l}
3 \\
3
\end{array}\right)\left(\begin{array}{l}
1 \\
2
\end{array}\right)=1 \quad \text { if } k=2 \\
A_{4} & =\left(\begin{array}{l}
4 \\
4
\end{array}\right)+\left(\begin{array}{l}
2 \\
4
\end{array}\right)\left(\begin{array}{l}
2 \\
3
\end{array}\right)-\left(\begin{array}{l}
3 \\
4
\end{array}\right)\left(\begin{array}{l}
1 \\
3
\end{array}\right)-\left(\begin{array}{l}
1 \\
4
\end{array}\right)\left(\begin{array}{l}
3 \\
3
\end{array}\right)=3 \quad \text { if } k=3 \\
A_{4} & =\left(\begin{array}{c}
4 \\
k+1
\end{array}\right)+\left(\begin{array}{l}
2 \\
k+1
\end{array}\right)\left(\begin{array}{l}
2 \\
k
\end{array}\right)+\left(\begin{array}{l}
4 \\
k
\end{array}\right)-\left(\begin{array}{c}
3 \\
k+1
\end{array}\right)\left(\begin{array}{l}
1 \\
k
\end{array}\right)-\left(\begin{array}{c}
1 \\
k+1
\end{array}\right)\left(\begin{array}{l}
3 \\
k
\end{array}\right) \\
& =\frac{1}{2} k(k-1) \quad \text { if } k \geq 4 .
\end{aligned}
$$

The proof is thereby completed.

Arguing as in the above lemma, one derives the following counting formula:

Lemma 5.9 Let $y_{1}, \ldots, y_{L}$ be L critical points of $K$ in $\mathbb{S}_{+}^{n}$ and let $\iota\left(y_{j}\right):=n-\operatorname{morse}\left(K, y_{j}\right)$. Assume that

$$
B_{1}:=\sum_{j=1}^{L}(-1)^{\iota\left(y_{j}\right)}=-k \quad \text { with } k \geq 0 .
$$

Then the number $L$ has to satisfy $L:=2 r+k$ (with $r \in \mathbb{N}_{0}$ ) and there are $r$ even numbers $\iota\left(y_{j}\right)$ 's and $r+k$ odd numbers $\iota\left(y_{j}\right)$ 's. Furthermore, it holds

$$
B_{2}:=\sum_{1 \leq j<\ell \leq L}(-1)^{\iota\left(y_{j}\right)+\iota\left(y_{\ell}\right)}=-r+\frac{1}{2} k(k-1) ; \text { for each } L \geq 0 .
$$




\section{References}

1. Ahmedou, M., Ben Ayed, M.: Non Simple Blow Ups for the Nirenberg Problem on Half Spheres, preprint (2020). arXiv:2012.11728

2. Ahmedou, M., Ben Ayed, M.: The Nirenberg Problem on Half Spheres: A Bubbling off Analysis. preprint (2021)

3. Aubin, T.: Equations différentielles non linéaires et problème de Yamabe concernant la courbure scalaire. J. Math. Pures Appl. (9) 55(3), 269-296 (1976)

4. Aubin, T., Hebey, E.: Courbure scalaire prescrite (French) [Prescribed scalar curvature]. Bull. Sci. Math. 115(2), 125-131 (1991)

5. Bahri, A.: Critical Points at Infinity in Some Variational Problems, Research Notes in Mathematics, 182. Longman-Pitman, London (1989)

6. Bahri, A.: An invariant for yamabe-type flows with applications to scalar curvature problems in high dimensions. A celebration of J. F. Nash Jr. Duke Math. J. 81, 323-466 (1996)

7. Bahri, A., Coron, J.-M.: The scalar curvature problem on the standard three dimensional spheres. J. Funct. Anal. 95, 106-172 (1991)

8. Bahri, A., Coron, J.-M.: On a nonlinear elliptic equation involving the critical Sobolev exponent: the effect of the topology of the domain. Commun. Pure Appl. Math. 41, 253-294 (1988)

9. Bahri, A., Li, Y.Y., Rey, O.: On a variational problem with lack of compactness: the topological effect of the critical points at infinity. Calc. Var. Partial Differ. Equ. 3, 67-93 (1995)

10. Brézis, H., Nirenberg, L.: Positive solutions of nonlinear elliptic equations involving critical Sobolev exponents. Commun. Pure Appl. Math. 36(4), 437-477 (1983)

11. Ben Ayed, M., Chen, Y., Chtioui, H., Hammami, M.: On the prescribed scalar curvature problem on 4-manifolds. Duke Math. J. 84, 633-677 (1996)

12. Ben Ayed, M., Chtioui, H., Hammami, M.: A Morse lemma at infinity for Yamabe type problems on domains. Ann. Inst. H. Poincaré Anal. Non Linéaire 20, 543-577 (2003)

13. Ben Ayed, M., El Mehdi, K., Ahmedou, M.O.: Prescribing the scalar curvature under minimal boundary conditions on the half sphere. Adv. Nonlinear Stud. 2(2), 93-116 (2002)

14. Ben Ayed, M., El Mehdi, K., Ould Ahmedou, M.: The scalar curvature problem on the four dimensional half sphere. Calc. Var. Partial Differ. Equ. 22(4), 465-482 (2005)

15. Ben Ayed, M., Ghoudi, R., Ould Bouh, K.: Existence of conformal metrics with prescribed scalar curvature on the four dimensional half sphere. NoDEA Nonlinear Differ. Equ. Appl. 19, 629-662 (2012)

16. Ben Ayed, M.: Bahri-Coron type theorem for the scalar curvature problem on high dimensional spheres. Ann. Mat. Pura Appl. 191, 95-112 (2012)

17. Ben Ayed, M., Ould Ahmedou, M.: On the prescribed scalar curvature on 3-half spheres: multiplicity results and Morse inequalities at infinity. Discrete Contin. Dyn. Syst. 23(3), 655-683 (2009)

18. Bourguignon, J.P., Ezin, J.P.: Scalar curvature functions in a conformal class of metrics and conformal transformations. Trans. Am. Math. Soc. 301, 723-736 (1987)

19. Chang, A., Yang, P.: A perturbation result in prescribing scalar curvature on $\mathbb{S}^{n}$. Duke Math. J. 64, 27-69 (1991)

20. Chang, A., Gursky, Matthew J., Yang, Paul C.: The scalar curvature equation on 2- and 3-spheres. Calc. Var. Partial Differ. Equ. 1(2), 205-229 (1993)

21. Chen, C.C., Lin, C.S.: Blowing up with infinite energy of conformal metrics on $\mathbb{S}^{n}$. Commun. Partial Differ. Equ. 24, 785-799 (1999)

22. Chen, C.C., Lin, C.S.: Prescribing the scalar curvature on $S^{n}$. I. Apriori estimates. J. Differ. Geom. 57, 67-171 (2001)

23. Chen, C.-C., Lin, C.S.: Estimate of the conformal scalar curvature equation via the method of moving planes II. J. Differ. Geom. 49, 115-178 (1998)

24. Chen, X., Xu, X.: The scalar curvature flow on $S^{n}$-perturbation theorem revisited. Invent. Math. 187(2), 395-506 (2012)

25. Djadli, Z., Malchiodi, A., Ould Ahmedou, M.: Prescribing scalar and boundary mean curvature on the three dimensional half sphere. J. Geom. Anal. 13, 255-289 (2003)

26. Dold, A.: Lectures on Algebraic Topology. Reprint of the 1972 edition. Classics in Mathematics. Springer, Berlin (1995)

27. Han, Z.-C.: Prescribing Gaussian curvature on $\mathbb{S}^{2}$. Duke Math. J. 61, 679-703 (1990)

28. Kazdan, J., Warner, F.: Existence and conformal deformation of metrics with prescribed Gaussian and scalar curvatures. Ann. Math. 2(101), 317-331 (1975)

29. Li, P.L., Liu, J.Q.: Nirenberg's problem on the two-dimensional hemi-sphere. Int. J. Math. 4, 927-939 (1993) 
30. Li, Y.Y.: The Nirenberg problem in a domain with boundary. Top. Methods Nonlinear Anal. 6, 309-329 (1995)

31. Li, Y.Y.: Prescribing scalar curvature on $S^{n}$ and related topics. Part I. J. Differ. Equ. 120, 319-410 (1995)

32. Li, Y.Y.: Prescribing scalar curvature on $S^{n}$ and related topics, Part II : existence and compactness. Commun. Pure Appl. Math. 49, 437-477 (1996)

33. Lions, P.L.: The concentration-compactness principle in the calculus of variations. The limit case Part I. Rev. Mat. Iberoamericano 1, 145-201 (1985)

34. Malchiodi, A., Mayer, M.: Prescribing Morse Scalar Curvatures: Blow-up Analysis. preprint (2019)

35. Malchiodi, A., Mayer, M.: Prescribing Morse Scalar Curvatures: Pinching and Morse Theory. Preprint (2019)

36. Schoen, R.: Topics in Differential Geometry, Graduate Course at Stanford University (1988). http://sites. math.washington.edu/ pollack/research/Pollack-notes-Schoen1988.pdf

37. Schoen, R.: Zhang, Dong: Prescribed scalar curvature on the n-sphere. Calc. Var. Partial Differ. Equ. 4, $1-25$ (1996)

38. Struwe, M.: A global compactness result for elliptic boundary value problems involving limiting nonlinearities. Math. Z. 187, 511-517 (1984)

Publisher's Note Springer Nature remains neutral with regard to jurisdictional claims in published maps and institutional affiliations. 